Included in: Combinatorial and Additive Number Theory: CANT 2011 and 2012 (edited by M.B. Nathanson), Springer Proc. in Math. \& Stat., Vol. 101, Springer, New York, 2014, pp. 257-312.

\title{
ON SUMS RELATED TO CENTRAL BINOMIAL AND TRINOMIAL COEFFICIENTS
}

\author{
ZHI-WEI SUN
}

\begin{abstract}
A generalized central trinomial coefficient $T_{n}(b, c)$ is the coefficient of $x^{n}$ in the expansion of $\left(x^{2}+b x+c\right)^{n}$ with $b, c \in \mathbb{Z}$. In this paper we investigate congruences and series for sums of terms related to central binomial coefficients and generalized central trinomial coefficients. The paper contains many conjectures on congruences related to representations of primes by certain binary quadratic forms, and 62 proposed new series for $1 / \pi$ motivated by congruences and related dualities.
\end{abstract}

\section{InTRODUCTION}

Let $\mathbb{N}=\{0,1,2, \ldots\}$. The central binomial coefficients

$$
\left(\begin{array}{c}
2 n \\
n
\end{array}\right)=\frac{(2 n) !}{(n !)^{2}} \quad(n \in \mathbb{N})
$$

play important roles in combinatorics and number theory. In this section we first review some known results on sums involving products of at most three central binomial coefficients.

Let $\mathbb{Z}^{+}=\{1,2,3, \ldots\}$. Recall that for given numbers $A$ and $B$ the Lucas sequence $u_{n}=u_{n}(A, B)(n \in \mathbb{N})$ and its companion $v_{n}=$ $v_{n}(A, B)(n \in \mathbb{N})$ are defined by

$$
u_{0}=0, u_{1}=1, u_{n+1}=A u_{n}-B u_{n-1}\left(n \in \mathbb{Z}^{+}\right),
$$

and

$$
v_{0}=2, v_{1}=A, v_{n+1}=A v_{n}-B v_{n-1}\left(n \in \mathbb{Z}^{+}\right) .
$$

It is well known that

$$
(\alpha-\beta) u_{n}=\alpha^{n}-\beta^{n} \text { and } v_{n}=\alpha^{n}+\beta^{n} \text { for all } n \in \mathbb{N} \text {, }
$$

where $\alpha=(A+\sqrt{\Delta}) / 2$ and $\beta=(A-\sqrt{\Delta}) / 2$ with $\Delta=A^{2}-4 B$.

Key words and phrases. Central binomial coefficients, central trinomial coefficients, congruences, representations of primes by binary quadratic forms, series for $1 / \pi$

2010 Mathematics Subject Classification. Primary 11B65, 11E25; Secondary 05A10, 11A07, 33F05. 
Let $p$ be an odd prime and let $m$ be any integer not divisible by $p$. The author [Su1] proved that

$$
\sum_{k=0}^{p-1} \frac{\left(\begin{array}{c}
2 k \\
k
\end{array}\right)}{m^{k}} \equiv\left(\frac{m^{2}-4 m}{p}\right)+u_{p-\left(\frac{m^{2}-4 m}{p}\right)}(m-2,1)\left(\bmod p^{2}\right),
$$

where $(-)$ denotes the Jacobi symbol.

Let $p \equiv 1(\bmod 4)$ be a prime. Write $p=x^{2}+y^{2}$ with $x \equiv 1(\bmod 4)$ and $y \equiv 0(\bmod 2)$. Gauss showed in 1828 the famous congruence

$$
\left(\begin{array}{c}
(p-1) / 2 \\
(p-1) / 4
\end{array}\right) \equiv 2 x(\bmod p)
$$

and this was further refined by S. Chowla, B. Dwork and R. J. Evans [CDE] in 1986 who used Gauss and Jacobi sums to show that

$$
\left(\begin{array}{l}
(p-1) / 2 \\
(p-1) / 4
\end{array}\right) \equiv \frac{2^{p-1}+1}{2}\left(2 x-\frac{p}{2 x}\right)\left(\bmod p^{2}\right) .
$$

For more such congruences involving products of one or more binomial coefficients, the reader may consult the excellent survey [HW] by R. H. Hudson and K. S. Williams. In 2009 the author (cf. [Su2, Conjecture 5.5]) conjectured that

$\sum_{k=0}^{p-1} \frac{\left(\begin{array}{c}2 k \\ k\end{array}\right)^{2}}{8^{k}} \equiv \sum_{k=0}^{p-1} \frac{\left(\begin{array}{c}2 k \\ k\end{array}\right)^{2}}{(-16)^{k}} \equiv\left(\frac{2}{p}\right) \sum_{k=0}^{p-1} \frac{\left(\begin{array}{c}2 k \\ k\end{array}\right)^{2}}{32^{k}} \equiv\left(\frac{2}{p}\right)\left(2 x-\frac{p}{2 x}\right)\left(\bmod p^{2}\right)$,

and this was later confirmed by Z.-H. Sun [S1]. Recently the author [Su5] determined $x \bmod p^{2}$ via the congruence

$$
\left(\frac{2}{p}\right) x \equiv \sum_{k=0}^{(p-1) / 2} \frac{k+1}{8^{k}}\left(\begin{array}{c}
2 k \\
k
\end{array}\right)^{2} \equiv \sum_{k=0}^{(p-1) / 2} \frac{2 k+1}{(-16)^{k}}\left(\begin{array}{c}
2 k \\
k
\end{array}\right)^{2}\left(\bmod p^{2}\right) .
$$

Note that $p \mid\left(\begin{array}{c}2 k \\ k\end{array}\right)$ for all $k=(p+1) / 2, \ldots, p-1$.

Let $p$ be an odd prime. By [I, vH],

$$
\sum_{k=0}^{p-1} \frac{\left(\begin{array}{c}
2 k \\
k
\end{array}\right)^{3}}{64^{k}} \equiv \begin{cases}4 x^{2}-2 p\left(\bmod p^{2}\right) & \text { if } p=x^{2}+y^{2}(2 \nmid x \& 2 \mid y), \\
0\left(\bmod p^{2}\right) & \text { if } p \equiv 3(\bmod 4) .\end{cases}
$$

In $[\mathrm{Su} 2, \mathrm{Su} 4]$ the author made conjectures on $\sum_{k=0}^{p-1}\left(\begin{array}{c}2 k \\ k\end{array}\right)^{3} / m^{k} \bmod p^{2}$ for $m=1,-8,16,-64,256,-512,4096$; for example, Conjecture 5.3 of [Su2] states that

$$
\sum_{k=0}^{p-1}\left(\begin{array}{c}
2 k \\
k
\end{array}\right)^{3} \equiv \begin{cases}4 x^{2}-2 p\left(\bmod p^{2}\right) & \text { if }\left(\frac{p}{7}\right)=1 \& p=x^{2}+7 y^{2} \\
0\left(\bmod p^{2}\right) & \text { if }\left(\frac{p}{7}\right)=-1\end{cases}
$$


(Throughout this paper, when we write a multiple of a prime in the form $a x^{2}+b y^{2}$, we always assume that $x$ and $y$ are nonzero integers.) To attack such conjectures, Z.-H. Sun [S2] deduced the useful combinatorial identity

$$
\sum_{k=0}^{n}\left(\begin{array}{c}
n+k \\
2 k
\end{array}\right)\left(\begin{array}{c}
2 k \\
k
\end{array}\right)^{2} x^{k}=P_{n}(\sqrt{1+4 x})^{2}
$$

where $P_{n}(x)$ is the Legendre polynomial of degree $n$ given by

$$
P_{n}(x):=\sum_{k=0}^{n}\left(\begin{array}{l}
n \\
k
\end{array}\right)\left(\begin{array}{c}
n+k \\
k
\end{array}\right)\left(\frac{x-1}{2}\right)^{k} .
$$

Actually (1) is just a special case of the well-known Clausen formula for hypergeometric series. We can rewrite (1) in the form

$$
\sum_{k=0}^{n}\left(\begin{array}{l}
n \\
k
\end{array}\right)\left(\begin{array}{c}
n+k \\
k
\end{array}\right)\left(\begin{array}{c}
2 k \\
k
\end{array}\right)(x(x+1))^{k}=D_{n}(x)^{2}
$$

where $D_{n}(x)$ is the Delannoy polynomial of degree $n$ given by

$$
D_{n}(x):=\sum_{k=0}^{n}\left(\begin{array}{l}
n \\
k
\end{array}\right)\left(\begin{array}{c}
n+k \\
k
\end{array}\right) x^{k}
$$

Note that those $D_{n}=D_{n}(1)(n=0,1,2, \ldots)$ are central Delannoy numbers (see, e.g., [CHV], [Su3] and [St, p. 178]. It is well known that $P_{n}(-x)=(-1)^{n} P_{n}(x)$, i.e., $(-1)^{n} D_{n}(x)=D_{n}(-x-1)$ (cf. [Su3, Remark 1.2]). As observed by Z.-H. Sun [S1, Lemma 2.2], if $0 \leqslant k \leqslant$ $n=(p-1) / 2$ then

$$
\left(\begin{array}{c}
n+k \\
2 k
\end{array}\right) \equiv \frac{\left(\begin{array}{c}
2 k \\
k
\end{array}\right)}{(-16)^{k}}\left(\bmod p^{2}\right)
$$

and hence

$$
\left(\begin{array}{l}
n \\
k
\end{array}\right)\left(\begin{array}{c}
n+k \\
k
\end{array}\right)=\left(\begin{array}{c}
n+k \\
2 k
\end{array}\right)\left(\begin{array}{c}
2 k \\
k
\end{array}\right) \equiv \frac{\left(\begin{array}{c}
2 k \\
k
\end{array}\right)^{2}}{(-16)^{k}}\left(\bmod p^{2}\right) .
$$

This simple trick was also realized by van Hamme [vH, p. 231]. Combining this useful trick with the identity (2), we see that

$$
\sum_{k=0}^{p-1} \frac{\left(\begin{array}{c}
2 k \\
k
\end{array}\right)^{3}}{(-16)^{k}}(x(x+1))^{k} \equiv\left(\sum_{k=0}^{p-1} \frac{\left(\begin{array}{c}
2 k \\
k
\end{array}\right)^{2}}{(-16)^{k}} x^{k}\right)^{2}\left(\bmod p^{2}\right) .
$$

To study the author's conjectures on

$$
\sum_{k=0}^{p-1} \frac{\left(\begin{array}{c}
2 k \\
k
\end{array}\right)^{2}\left(\begin{array}{c}
3 k \\
k
\end{array}\right)}{m^{k}}, \sum_{k=0}^{p-1} \frac{\left(\begin{array}{c}
2 k \\
k
\end{array}\right)^{2}\left(\begin{array}{c}
4 k \\
2 k
\end{array}\right)}{m^{k}}, \sum_{k=0}^{p-1} \frac{\left(\begin{array}{c}
2 k \\
k
\end{array}\right)\left(\begin{array}{c}
3 k \\
k
\end{array}\right)\left(\begin{array}{c}
6 k \\
3 k
\end{array}\right)}{m^{k}}
$$


modulo $p^{2}$ (with $m$ suitable integers not divisible by $p$ ) given in [Su4, Su7], Z.-H. Sun [S2, S3, S4] managed to prove the following congruences similar to (3):

$$
\begin{aligned}
& \sum_{k=0}^{p-1} \frac{\left(\begin{array}{c}
2 k \\
k
\end{array}\right)^{2}\left(\begin{array}{c}
4 k \\
2 k
\end{array}\right)}{(-64)^{k}}(x(x+1))^{k} \equiv\left(\sum_{k=0}^{p-1} \frac{\left(\begin{array}{c}
4 k \\
2 k
\end{array}\right)\left(\begin{array}{c}
2 k \\
k
\end{array}\right)}{(-64)^{k}} x^{k}\right)^{2}\left(\bmod p^{2}\right), \\
& \sum_{k=0}^{p-1} \frac{\left(\begin{array}{c}
2 k \\
k
\end{array}\right)^{2}\left(\begin{array}{c}
3 k \\
k
\end{array}\right)}{(-27)^{k}}(x(x+1))^{k} \equiv\left(\sum_{k=0}^{p-1} \frac{\left(\begin{array}{c}
2 k \\
k
\end{array}\right)\left(\begin{array}{c}
3 k \\
k
\end{array}\right)}{(-27)^{k}} x^{k}\right)^{2}\left(\bmod p^{2}\right)(p>3), \\
& \sum_{k=0}^{p-1} \frac{\left(\begin{array}{c}
2 k \\
k
\end{array}\right)\left(\begin{array}{c}
3 k \\
k
\end{array}\right)\left(\begin{array}{c}
6 k \\
3 k
\end{array}\right)}{(-432)^{k}}(x(x+1))^{k} \equiv\left(\sum_{k=0}^{p-1} \frac{\left(\begin{array}{c}
6 k \\
3 k
\end{array}\right)\left(\begin{array}{c}
3 k \\
k
\end{array}\right)}{(-432)^{k}} x^{k}\right)^{2}\left(\bmod p^{2}\right)(p>3) .
\end{aligned}
$$

In 1859 G. Bauer proved that

$$
\sum_{k=0}^{\infty}(4 k+1) \frac{\left(\begin{array}{c}
2 k \\
k
\end{array}\right)^{3}}{(-64)^{k}}=\frac{2}{\pi}
$$

In $1914 \mathrm{~S}$. Ramanujan [R] found 16 new series for $1 / \pi$ which are quite similar to Bauer's series. The (rational) Ramanujan-type series for $1 / \pi$ (cf. B. C. Berndt [Be, pp. 353-354], and also [BB] and [ChCh]) have the following form:

$$
\sum_{k=0}^{\infty}(a+d k) \frac{f(k)}{m^{k}}=\frac{C}{\pi}
$$

where $f(k)$ refers to one of

$$
\left(\begin{array}{c}
2 k \\
k
\end{array}\right)^{3},\left(\begin{array}{c}
2 k \\
k
\end{array}\right)^{2}\left(\begin{array}{c}
3 k \\
k
\end{array}\right),\left(\begin{array}{c}
2 k \\
k
\end{array}\right)^{2}\left(\begin{array}{c}
4 k \\
2 k
\end{array}\right),\left(\begin{array}{c}
2 k \\
k
\end{array}\right)\left(\begin{array}{c}
3 k \\
k
\end{array}\right)\left(\begin{array}{c}
6 k \\
k
\end{array}\right),
$$

and $a, d, m$ are integers with $d m \neq 0$, and $C^{2}$ is rational. Up to now, 36 such series have been established via the theory of modular forms. The reader may consult [CCL], [CC], and S. Cooper [C] for some other series for $1 / \pi$.

Let $p$ be an odd prime. Note that $\Gamma(1 / 2)^{2}=\pi$ while $\Gamma_{p}(1 / 2)^{2}=$ $(-1)^{(p+1) / 2}$, where $\Gamma_{p}(x)$ denotes the $p$-adic $\Gamma$-function. In view of this, in 1997 van Hamme [vH] studied $p$-adic supercongruences for partial sums of some hypergeometric series involving the Gamma function. (If a $p$-adic congruence happens to be true modulo a higher power of $p$, then it is called a supercongruence.) For example, Bauer's series led 
him to conjecture that

$$
\sum_{k=0}^{p-1}(4 k+1) \frac{\left(\begin{array}{c}
2 k \\
k
\end{array}\right)^{3}}{(-64)^{k}} \equiv p\left(\frac{-1}{p}\right)\left(\bmod p^{3}\right)
$$

which was later confirmed by E. Mortenson [M2] in 2008. More supercongruences motivated by Ramanujan-type series have been investigated by some followers of van Hamme, see, e.g., L. Long [L] and Zudilin's work stated there. The author [Su6] refined the congruence by van Hamme and Mortenson to the following congruence $\bmod p^{4}$ :

$\sum_{k=0}^{p-1}(4 k+1) \frac{\left(\begin{array}{c}2 k \\ k\end{array}\right)^{3}}{(-64)^{k}} \equiv \sum_{k=0}^{(p-1) / 2}(4 k+1) \frac{\left(\begin{array}{c}2 k \\ k\end{array}\right)^{3}}{(-64)^{k}} \equiv p\left(\frac{-1}{p}\right)+p^{3} E_{p-3}\left(\bmod p^{4}\right)$,

where $E_{0}, E_{1}, \ldots$ are the Euler numbers defined by

$$
E_{0}=1, \text { and } \sum_{\substack{k=0 \\
2 \mid k}}^{n}\left(\begin{array}{l}
n \\
k
\end{array}\right) E_{n-k}=0 \quad\left(n \in \mathbb{Z}^{+}\right)
$$

For more conjectural connections between Ramanujan-type congruences and Euler numbers or Euler polynomials, the reader may consult [Su4].

Gosper announced in 1974 that $\sum_{k=0}^{\infty}(25 k-3) /\left(2^{k}\left(\begin{array}{c}3 k \\ k\end{array}\right)\right)=\pi / 2$ (see G. Almkvist, C. Krattenthaler and J. Petersson $[\mathrm{AKP}]$ for a simple proof). Though this is not a Ramanujan-type series, the author conjectures that for any prime $p>3$ we have

$$
\begin{aligned}
2 p \sum_{k=0}^{p-1} \frac{25 k-3}{2^{k}\left(\begin{array}{c}
3 k \\
k
\end{array}\right)} & \equiv 3\left(\frac{-1}{p}\right)+\left(E_{p-3}-9\right) p^{2}\left(\bmod p^{3}\right), \\
p \sum_{k=0}^{(p-1) / 2} \frac{25 k-3}{2^{k}\left(\begin{array}{c}
3 k \\
k
\end{array}\right)} & \equiv\left(\frac{-1}{p}\right)-\left(\frac{2}{p}\right) \frac{5 p}{2}\left(\bmod p^{2}\right), \\
\sum_{k=0}^{p-1}(25 k+3) k 2^{k}\left(\begin{array}{c}
3 k \\
k
\end{array}\right) & \equiv 6\left(\frac{-1}{p}\right)-18 p\left(\bmod p^{2}\right) .
\end{aligned}
$$

The author [Su4] found some new series for powers of $\pi$ motivated by corresponding $p$-adic congruences. Here is a new example: Immediately 
after the author discovered the conjectural congruence

$$
\begin{gathered}
\sum_{n=0}^{p-1} \frac{28 n+5}{576^{n}}\left(\begin{array}{c}
2 n \\
n
\end{array}\right) \sum_{k=0}^{n} \frac{5^{k}\left(\begin{array}{c}
2 k \\
k
\end{array}\right)^{2}\left(\begin{array}{c}
2(n-k) \\
n-k
\end{array}\right)^{2}}{\left(\begin{array}{l}
n \\
k
\end{array}\right)} \\
\equiv p\left(\frac{-1}{p}\right)\left(3+2\left(\frac{2}{p}\right)\right)\left(\bmod p^{2}\right)
\end{gathered}
$$

for any prime $p>3$, he conjectured (on Jan. 14, 2012) that

$$
\sum_{n=0}^{\infty} \frac{28 n+5}{576^{n}}\left(\begin{array}{c}
2 n \\
n
\end{array}\right) \sum_{k=0}^{n} \frac{5^{k}\left(\begin{array}{c}
2 k \\
k
\end{array}\right)^{2}\left(\begin{array}{c}
2(n-k) \\
n-k
\end{array}\right)^{2}}{\left(\begin{array}{c}
n \\
k
\end{array}\right)}=\frac{9}{\pi}(2+\sqrt{2}) .
$$

The author [Su4, Su7] found that an identity like (7) usually corresponds to a congruence for $\sum_{k=0}^{p-1} f(k) / m^{k}$ modulo $p^{2}$ in terms of parameters in representations of a prime $p$ or its multiple by certain binary quadratic forms. This was the main starting point of the author's discoveries of many new series for $1 / \pi$.

Let $n \in \mathbb{N}$. Clearly $\left(\begin{array}{c}2 n \\ n\end{array}\right)$ is the coefficient of $x^{n}$ in the expansion of $\left(x^{2}+2 x+1\right)^{n}=(x+1)^{2 n}$. The $n$th central trinomial coefficient

$$
T_{n}=\left[x^{n}\right]\left(x^{2}+x+1\right)^{n}
$$

is the coefficient of $x^{n}$ in the expansion of $\left(x^{2}+x+1\right)^{n}$. Since $T_{n}$ is the constant term of $\left(1+x+x^{-1}\right)^{n}$, by the multi-nomial theorem we see that

$$
T_{n}=\sum_{k=0}^{\lfloor n / 2\rfloor} \frac{n !}{k ! k !(n-2 k) !}=\sum_{k=0}^{\lfloor n / 2\rfloor}\left(\begin{array}{c}
n \\
2 k
\end{array}\right)\left(\begin{array}{c}
2 k \\
k
\end{array}\right)=\sum_{k=0}^{n}\left(\begin{array}{l}
n \\
k
\end{array}\right)\left(\begin{array}{c}
n-k \\
k
\end{array}\right) .
$$

Central trinomial coefficients arise naturally in enumerative combinatorics (cf. [Sl]), e.g., $T_{n}$ is the number of lattice paths from the point $(0,0)$ to $(n, 0)$ with only allowed steps $(1,1),(1,-1)$ and $(1,0)$.

Given $b, c \in \mathbb{Z}$, we define the generalized central trinomial coefficients

$$
\begin{aligned}
T_{n}(b, c): & =\left[x^{n}\right]\left(x^{2}+b x+c\right)^{n}=\left[x^{0}\right]\left(b+x+c x^{-1}\right)^{n} \\
& =\sum_{k=0}^{\lfloor n / 2\rfloor}\left(\begin{array}{c}
n \\
2 k
\end{array}\right)\left(\begin{array}{c}
2 k \\
k
\end{array}\right) b^{n-2 k} c^{k}=\sum_{k=0}^{\lfloor n / 2\rfloor}\left(\begin{array}{c}
n \\
k
\end{array}\right)\left(\begin{array}{c}
n-k \\
k
\end{array}\right) b^{n-2 k} c^{k} .
\end{aligned}
$$

Clearly $T_{n}(2,1)=\left(\begin{array}{c}2 n \\ n\end{array}\right)$ and $T_{n}(1,1)=T_{n}$. An efficient way to compute $T_{n}(b, c)$ is to use the initial values $T_{0}(b, c)=1$ and $T_{1}(b, c)=b$, and the recursion

$(n+1) T_{n+1}(b, c)=(2 n+1) b T_{n}(b, c)-n\left(b^{2}-4 c\right) T_{n-1}(b, c) \quad(n=1,2, \ldots)$.

Note that the recursion is rather simple if $b^{2}-4 c=0$. 
Let $b, c \in \mathbb{Z}$ and $d=b^{2}-4 c$. It is known that $T_{n}(b, c)=\sqrt{d}^{n} P_{n}(b / \sqrt{d})$ if $d \neq 0$ (see, e.g., [N] and [Su9]). Thus

$$
T_{n}(b, c)=\sum_{k=0}^{n}\left(\begin{array}{c}
n+k \\
2 k
\end{array}\right)\left(\begin{array}{c}
2 k \\
k
\end{array}\right)\left(\frac{b-\sqrt{d}}{2}\right)^{k} \sqrt{d}^{n-k} .
$$

(In the case $d=0,(10)$ holds trivially since $x^{2}+b x+c=(x+b / 2)^{2}$.) By the Laplace-Heine formula (cf. [Sz, p. 194]), for any complex number $x \notin[-1,1]$ we have

$$
P_{n}(x) \sim \frac{\left(x+\sqrt{x^{2}-1}\right)^{n+1 / 2}}{\sqrt{2 n \pi} \sqrt[4]{x^{2}-1}} \quad \text { as } n \rightarrow+\infty .
$$

It follows that if $b>0$ and $c>0$ then

$$
T_{n}(b, c) \sim f_{n}(b, c):=\frac{(b+2 \sqrt{c})^{n+1 / 2}}{2 \sqrt[4]{c} \sqrt{n \pi}} \text { as } n \rightarrow+\infty .
$$

Note that $T_{n}(-b, c)=(-1)^{n} T_{n}(b, c)$.

The generalized central trinomial coefficients seem to be natural extensions of the central binomial coefficients. To see this, in the next section we study congruences for

$$
\sum_{k=0}^{p-1} \frac{\left(\begin{array}{c}
2 k \\
k
\end{array}\right) T_{k}(b, c)}{m^{k}} \text { and } \sum_{k=0}^{p-1} \frac{\left(\begin{array}{c}
2 k \\
k
\end{array}\right) T_{2 k}(b, c)}{m^{k}}
$$

modulo an odd prime $p$, where $b, c, m \in \mathbb{Z}$ and $m \not \equiv 0(\bmod p)$. One may compare them with congruences for $\sum_{k=0}^{p-1}\left(\begin{array}{c}2 k \\ k\end{array}\right)^{2} / m^{k} \bmod p^{2}$ with $m=8,-16,32$. Since

$$
T_{k}(2,1)=\left(\begin{array}{c}
2 k \\
k
\end{array}\right), T_{2 k}(2,1)=\left(\begin{array}{c}
4 k \\
2 k
\end{array}\right) \text { and } T_{3 k}(2,1)=\left(\begin{array}{c}
6 k \\
3 k
\end{array}\right),
$$

in Section 3 we are going to investigate general sums

$$
\sum_{k=0}^{p-1} \frac{\left(\begin{array}{c}
2 k \\
k
\end{array}\right)^{2}}{m^{k}} T_{k}(b, c), \sum_{k=0}^{p-1} \frac{\left(\begin{array}{c}
2 k \\
k
\end{array}\right)\left(\begin{array}{c}
3 k \\
k
\end{array}\right)}{m^{k}} T_{k}(b, c), \sum_{k=0}^{p-1} \frac{\left(\begin{array}{c}
4 k \\
2 k
\end{array}\right)\left(\begin{array}{c}
2 k \\
k
\end{array}\right)}{m^{k}} T_{k}(b, c)
$$

and

$$
\sum_{k=0}^{p-1} \frac{\left(\begin{array}{c}
2 k \\
k
\end{array}\right)^{2}}{m^{k}} T_{2 k}(b, c), \sum_{k=0}^{p-1} \frac{\left(\begin{array}{c}
2 k \\
k
\end{array}\right)\left(\begin{array}{c}
3 k \\
k
\end{array}\right)}{m^{k}} T_{3 k}(b, c)
$$

modulo $p^{2}$, where $p$ is an odd prime, $b, c, m \in \mathbb{Z}$ and $m \not \equiv 0(\bmod p)$. For this purpose, we need to extend those congruences (3)-(6) in Section 3.

Section 4 contains many conjectural congruences involving generalized central binomial coefficients and they offer backgrounds for those 
conjectural series for $1 / \pi$ in Sect. 5 . In the fifth section we first show a theorem on dualities and then propose 61 new conjectural series for $1 / \pi$ based on our investigation of congruences.

2. ON $\sum_{k=0}^{p-1}\left(\begin{array}{c}2 k \\ k\end{array}\right) T_{k}(b, c) / m^{k}$ AND $\sum_{k=0}^{p-1}\left(\begin{array}{c}2 k \\ k\end{array}\right) T_{2 k}(b, c) / m^{k}$ MODULO $p$

Lemma 2.1. Let $p=2 n+1$ be an odd prime and let $k \in\{0, \ldots, n\}$. Then

$$
\left(\begin{array}{c}
2 k \\
k
\end{array}\right) \equiv(-1)^{n} 16^{k}\left(\begin{array}{c}
2(n-k) \\
n-k
\end{array}\right)(\bmod p) .
$$

Given $b, c \in \mathbb{Z}$ with $b^{2} \not \equiv 4 c(\bmod p)$, we also have

$$
T_{2(n-k)}(b, c) \equiv\left(\frac{b^{2}-4 c}{p}\right) \frac{T_{2 k}(b, c)}{\left(b^{2}-4 c\right)^{2 k}}(\bmod p) .
$$

Proof. (12) holds because

$$
\frac{\left(\begin{array}{c}
2 k \\
k
\end{array}\right)}{(-4)^{k}}=\left(\begin{array}{c}
-1 / 2 \\
k
\end{array}\right) \equiv\left(\begin{array}{l}
n \\
k
\end{array}\right)=\left(\begin{array}{c}
n \\
n-k
\end{array}\right) \equiv\left(\begin{array}{c}
-1 / 2 \\
n-k
\end{array}\right)=\frac{\left(\begin{array}{c}
2(n-k) \\
n-k
\end{array}\right)}{(-4)^{n-k}}(\bmod p) .
$$

For $b, c \in \mathbb{Z}$ with $d=b^{2}-4 c \not \equiv 0(\bmod p)$, we get $(13)$ from the known result $d^{j} T_{p-1-j}(b, c) \equiv\left(\frac{d}{p}\right) T_{j}(b, c)$ for $j=0, \ldots, p-1$ (see $[\mathrm{N},(14)]$ or [Su9, Lemma 2.2]).

Theorem 2.1. Let $p$ be an odd prime and let $m, b, c \in \mathbb{Z}$ with $m \not \equiv$ $0(\bmod p)$. If $m \equiv 4 b(\bmod p)$, then

$$
\begin{aligned}
& \sum_{k=0}^{p-1} \frac{\left(\begin{array}{c}
2 k \\
k
\end{array}\right)}{m^{k}} T_{k}(b, c) \\
\equiv & \begin{cases}\left(\frac{m}{p}\right) 2 x c^{(p-1) / 4}(\bmod p) & \text { if } p=x^{2}+y^{2}(4 \mid x-1), \\
0(\bmod p) & \text { if } p \equiv 3(\bmod 4) .\end{cases}
\end{aligned}
$$

If $m \not \equiv 4 b(\bmod p)$, then

$$
\sum_{k=0}^{p-1} \frac{\left(\begin{array}{c}
2 k \\
k
\end{array}\right)}{m^{k}} T_{k}(b, c) \equiv\left(\frac{m(m-4 b)}{p}\right) \sum_{k=0}^{p-1} \frac{\left(\begin{array}{c}
4 k \\
2 k
\end{array}\right)\left(\begin{array}{c}
2 k \\
k
\end{array}\right) c^{k}}{(m-4 b)^{2 k}}(\bmod p) .
$$

Also, provided that $d=b^{2}-4 c \not \equiv 0(\bmod p)$, for any $h \in \mathbb{Z}^{+}$we have

$$
\sum_{k=0}^{p-1} \frac{\left(\begin{array}{c}
2 k \\
k
\end{array}\right)^{h} T_{2 k}(b, c)}{m^{k}} \equiv\left(\frac{(-1)^{h} d m}{p}\right) \sum_{k=0}^{p-1} \frac{\left(\begin{array}{c}
2 k \\
k
\end{array}\right)^{h} T_{2 k}(b, c)}{\left(16^{h} d^{2} / m\right)^{k}}(\bmod p) .
$$


Proof. Set $n=(p-1) / 2$. As $\left(\begin{array}{l}n \\ k\end{array}\right) \equiv\left(\begin{array}{c}-1 / 2 \\ k\end{array}\right)=\left(\begin{array}{c}2 k \\ k\end{array}\right) /(-4)^{k}(\bmod p)$ for all $k=0, \ldots, p-1$, we have

$$
\begin{aligned}
\sum_{k=0}^{p-1} \frac{\left(\begin{array}{c}
2 k \\
k
\end{array}\right)}{m^{k}} T_{k}(b, c) & \equiv \sum_{k=0}^{n}\left(\begin{array}{l}
n \\
k
\end{array}\right)\left(-\frac{4}{m}\right)^{k}\left[x^{0}\right]\left(x+b+c x^{-1}\right)^{k} \\
& =\left[x^{0}\right]\left(1-\frac{4}{m} \cdot \frac{x^{2}+b x+c}{x}\right)^{n} \\
& \equiv\left(\frac{m}{p}\right)\left[x^{n}\right]\left(m x-4\left(x^{2}+b x+c\right)\right)^{n} \\
& \equiv(-1)^{n}\left(\frac{m}{p}\right)\left[x^{n}\right]\left(x^{2}-\frac{m-4 b}{4} x+c\right)^{n} \\
& =\left(\frac{m}{p}\right) T_{n}\left(\frac{m-4 b}{4}, c\right)=\left(\frac{m}{p}\right) \frac{T_{n}(m-4 b, 16 c)}{2^{2 n}} \\
& \equiv\left(\frac{m}{p}\right) T_{n}(m-4 b, 16 c)(\bmod p) .
\end{aligned}
$$

Observe that

$$
\begin{aligned}
T_{n}(m-4 b, 16 c) & =\sum_{k=0}^{\lfloor n / 2\rfloor}\left(\begin{array}{c}
n \\
2 k
\end{array}\right)\left(\begin{array}{c}
2 k \\
k
\end{array}\right)(m-4 b)^{n-2 k}(16 c)^{k} \\
& \equiv \sum_{k=0}^{\lfloor n / 2\rfloor} \frac{\left(\begin{array}{c}
4 k \\
2 k
\end{array}\right)}{(-4)^{2 k}}\left(\begin{array}{c}
2 k \\
k
\end{array}\right)(m-4 b)^{n-2 k}(16 c)^{k} \\
& =\sum_{k=0}^{\lfloor n / 2\rfloor}\left(\begin{array}{c}
4 k \\
2 k
\end{array}\right)\left(\begin{array}{c}
2 k \\
k
\end{array}\right)(m-4 b)^{n-2 k} c^{k}(\bmod p) .
\end{aligned}
$$

Thus $(15)$ holds when $m \not \equiv 4 b(\bmod p)$. If $m \equiv 4 b(\bmod p)$, then

$$
T_{n}(m-4 b, 16 c) \equiv \begin{cases}\left(\begin{array}{c}
2 n \\
n
\end{array}\right)\left(\begin{array}{c}
n \\
n / 2
\end{array}\right) c^{n / 2}(\bmod p) & \text { if } 2 \mid n \\
0(\bmod p) & \text { if } 2 \nmid n .\end{cases}
$$

Clearly $\left(\begin{array}{c}2 n \\ n\end{array}\right)=\left(\begin{array}{c}p-1 \\ n\end{array}\right) \equiv(-1)^{n}(\bmod p)$. If $p=2 n+1 \equiv 1(\bmod 4)$ and $p=x^{2}+y^{2}$ with $x \equiv 1(\bmod 4)$, then $\left(\begin{array}{c}n \\ n / 2\end{array}\right) \equiv 2 x(\bmod p)$ as observed by Gauss. Thus, (14) holds when $m \equiv 4 b(\bmod p)$. 
Now suppose that $d=b^{2}-4 c \not \equiv 0(\bmod p)$ and $h \in \mathbb{Z}^{+}$. In view of Lemma 2.1, we have

$$
\begin{aligned}
\sum_{k=0}^{n} \frac{\left(\begin{array}{c}
2 k \\
k
\end{array}\right)^{h} T_{2 k}(b, c)}{m^{k}} & \equiv \sum_{k=0}^{n} \frac{\left((-1)^{n} 16^{k}\left(\begin{array}{c}
2(n-k) \\
n-k
\end{array}\right)\right)^{h}}{m^{k}}\left(\frac{d}{p}\right) d^{2 k} T_{2(n-k)}(b, c) \\
& =(-1)^{h n}\left(\frac{d}{p}\right) \sum_{j=0}^{n}\left(\frac{16^{h} d^{2}}{m}\right)^{n-j}\left(\begin{array}{c}
2 j \\
j
\end{array}\right)^{h} T_{2 j}(b, c) \\
& \equiv\left(\frac{(-1)^{h} d m}{p}\right) \sum_{k=0}^{n} \frac{\left(\begin{array}{c}
2 k \\
k
\end{array}\right)^{h} T_{2 k}(b, c)}{\left(16^{h} d^{2} / m\right)^{k}}(\bmod p) .
\end{aligned}
$$

Recall that $p \mid\left(\begin{array}{c}2 k \\ k\end{array}\right)$ for each $k=n+1, \ldots, p-1$. So (16) follows.

Corollary 2.1. Let $p$ be an odd prime. Then

$$
\begin{aligned}
& \sum_{k=0}^{p-1} \frac{\left(\begin{array}{c}
2 k \\
k
\end{array}\right)}{4^{k}} T_{k}(1,2) \\
\equiv & \begin{cases}(-1)^{(x-1) / 2+y / 4} 2 x(\bmod p) & \text { if } 8 \mid p-1 \& p=x^{2}+y^{2}(2 \nmid x), \\
(-1)^{(y-2) / 4} 2 y(\bmod p) & \text { if } 8 \mid p-5 \& p=x^{2}+y^{2}(2 \mid y), \\
0(\bmod p) & \text { if } p \equiv 3(\bmod 4) .\end{cases}
\end{aligned}
$$

Proof. If $p \equiv 3(\bmod 4)$, then $\sum_{k=0}^{p-1}\left(\begin{array}{c}2 k \\ k\end{array}\right) T_{k}(1,2) / 4^{k} \equiv 0(\bmod p)$ by (14) with $m=4, b=1$ and $c=2$.

Now assume that $p \equiv 1(\bmod 4)$ and write $p=x^{2}+y^{2}$ with $x \equiv$ $1(\bmod 4)$ and $y \equiv 0(\bmod 2)$. Applying $(14)$ with $m=4, b=1$ and $c=2$, we get

$$
\sum_{k=0}^{p-1} \frac{\left(\begin{array}{c}
2 k \\
k
\end{array}\right)}{4^{k}} T_{k}(1,2) \equiv 2 x \times 2^{(p-1) / 4}(\bmod p) .
$$

By Exercise 27 of [IR, p. 64] (an observation of Dirichlet),

$$
2^{(p-1) / 4} \equiv\left(\frac{y}{x}\right)^{x y / 2}(\bmod p)
$$

Note that

$$
\left(\frac{y}{x}\right)^{2}=\frac{y^{2}}{x^{2}} \equiv-1(\bmod p) \text { and hence }\left(\frac{y}{x}\right)^{4} \equiv 1(\bmod 4) .
$$

So we have

$$
2^{(p-1) / 4} \equiv\left(\frac{y}{x}\right)^{y / 2}(\bmod p)
$$


If $p \equiv 1(\bmod 8)$, then $4 \mid y$ and hence $2^{(p-1) / 4} \equiv(-1)^{y / 4}(\bmod p)$. If $p \equiv 5(\bmod 8)$, then $y \equiv 2(\bmod 4)$ and hence

$$
2^{(p-1) / 4} \equiv\left(\frac{y}{x}\right)^{2(y-2) / 4} \frac{y}{x} \equiv(-1)^{(y-2) / 4} \frac{y}{x}(\bmod p) .
$$

Combining the above, we obtain the desired result.

Corollary 2.2. For any prime $p>3$ we have

$$
\sum_{k=0}^{p-1} \frac{\left(\begin{array}{c}
2 k \\
k
\end{array}\right) T_{k}}{(-4)^{k}} \equiv\left(\frac{-1}{p}\right)(\bmod p) \quad \text { and } \sum_{k=0}^{p-1} \frac{\left(\begin{array}{c}
2 k \\
k
\end{array}\right) T_{k}}{12^{k}} \equiv\left(\frac{p}{3}\right)(\bmod p)
$$

Proof. Applying (15) with $b=c=1$ and $m \in\{-4,12\}$ we obtain

$$
\sum_{k=0}^{p-1} \frac{\left(\begin{array}{c}
2 k \\
k
\end{array}\right) T_{k}}{(-4)^{k}} \equiv\left(\frac{(-4)(-8)}{p}\right) \sum_{k=0}^{p-1} \frac{\left(\begin{array}{c}
4 k \\
2 k
\end{array}\right)\left(\begin{array}{c}
2 k \\
k
\end{array}\right)}{64^{k}}(\bmod p)
$$

and

$$
\sum_{k=0}^{p-1} \frac{\left(\begin{array}{c}
2 k \\
k
\end{array}\right) T_{k}}{12^{k}} \equiv\left(\frac{12 \times 8}{p}\right) \sum_{k=0}^{p-1} \frac{\left(\begin{array}{c}
4 k \\
2 k
\end{array}\right)\left(\begin{array}{c}
2 k \\
k
\end{array}\right)}{64^{k}}(\bmod p)
$$

It is known that

$$
\sum_{k=0}^{p-1} \frac{\left(\begin{array}{c}
4 k \\
2 k
\end{array}\right)\left(\begin{array}{c}
2 k \\
k
\end{array}\right)}{64^{k}} \equiv\left(\frac{-2}{p}\right)\left(\bmod p^{2}\right)
$$

which was conjectured in [RV] and proved in [M1]. So the two congruences in Corollary 2.2 are valid.

Theorem 2.2. Let $p$ be an odd prime. Then

$$
\sum_{k=0}^{p-1} \frac{\left(\begin{array}{c}
2 k \\
k
\end{array}\right) T_{2 k}}{4^{k}} \equiv\left(\frac{-2}{p}\right)(\bmod p)
$$


Proof. Set $n=(p-1) / 2$. Then

$$
\begin{aligned}
\sum_{k=0}^{p-1} \frac{\left(\begin{array}{c}
2 k \\
k
\end{array}\right)}{4^{k}} T_{2 k} & \equiv \sum_{k=0}^{n}\left(\begin{array}{l}
n \\
k
\end{array}\right)(-1)^{k}\left[x^{0}\right]\left(1+x+x^{-1}\right)^{2 k} \\
& =\left[x^{0}\right]\left(1-\left(1+x+x^{-1}\right)^{2}\right)^{n} \\
& =\left[x^{0}\right](-1)^{n}\left(\frac{x^{2}+1}{x} \cdot \frac{(x+1)^{2}}{x}\right)^{n} \\
& =(-1)^{n}\left[x^{2 n}\right]\left(x^{2}+1\right)^{n}(x+1)^{2 n}=(-1)^{n} \sum_{k=0}^{n}\left(\begin{array}{l}
n \\
k
\end{array}\right)\left(\begin{array}{l}
2 n \\
2 k
\end{array}\right) \\
& \equiv \sum_{k=0}^{n}\left(\begin{array}{l}
n \\
k
\end{array}\right)(-1)^{n}=(-2)^{n} \equiv\left(\frac{-2}{p}\right)(\bmod p) .
\end{aligned}
$$

This concludes the proof.

Remark 2.1. For any prime $p>3$ we observe the following congruences:

$$
\begin{aligned}
& \sum_{k=0}^{p-1} \frac{\left(\begin{array}{c}
2 k \\
k
\end{array}\right)}{4^{k}} T_{2 k}(5,4) \equiv 1(\bmod p), \quad \sum_{k=0}^{p-1} \frac{\left(\begin{array}{c}
2 k \\
k
\end{array}\right)}{4^{k}} T_{2 k}(3,1) \equiv\left(\frac{2}{p}\right)(\bmod p), \\
& \sum_{k=0}^{p-1} \frac{\left(\begin{array}{c}
2 k \\
k
\end{array}\right)}{16^{k}} T_{2 k}(4,9) \equiv\left(\frac{p}{3}\right)(\bmod p), \sum_{k=0}^{p-1} \frac{\left(\begin{array}{c}
2 k \\
k
\end{array}\right)}{16^{k}} T_{2 k}(8,25) \equiv\left(\frac{-5}{p}\right)(\bmod p) .
\end{aligned}
$$

Conjecture 2.1. Let $p>3$ be a prime. Then

$$
\begin{aligned}
\sum_{k=0}^{p-1} \frac{\left(\begin{array}{c}
2 k \\
k
\end{array}\right)}{12^{k}} T_{k} & \equiv\left(\frac{p}{3}\right) \frac{3^{p-1}+3}{4}\left(\bmod p^{2}\right), \\
\sum_{k=0}^{(p-1) / 2} \frac{\left(\begin{array}{c}
2 k \\
k
\end{array}\right)}{16^{k}} T_{2 k}(4,1) & \equiv 1\left(\bmod p^{2}\right), \\
\sum_{k=0}^{(p-1) / 2} \frac{\left(\begin{array}{c}
2 k \\
k
\end{array}\right)}{(k+1) 16^{k}} T_{2 k}(4,1) & \equiv \frac{4}{3}\left(\left(\frac{3}{p}\right)-p\left(\frac{-1}{p}\right)\right)\left(\bmod p^{2}\right), \\
\sum_{k=0}^{(p-1) / 2} \frac{\left(\begin{array}{c}
2 k \\
k
\end{array}\right)}{4^{k}} T_{2 k}(3,4) & \equiv\left(\frac{-1}{p}\right) \frac{7-3^{p}}{4}\left(\bmod p^{2}\right), \\
\sum_{k=0}^{(p-1) / 2} \frac{\left(\begin{array}{c}
2 k \\
k
\end{array}\right)}{16^{k}} T_{2 k}(8,9) & \equiv\left(\frac{3}{p}\right)\left(\bmod p^{2}\right),
\end{aligned}
$$


and

$$
\sum_{k=0}^{p-1} \frac{\left(\begin{array}{c}
3 k \\
k
\end{array}\right)}{432^{k}} T_{3 k}(6,1) \equiv 1(\bmod p) .
$$

Conjecture 2.2. Let $p>3$ be a prime. Then

$$
\begin{aligned}
& \sum_{k=0}^{(p-1) / 2} \frac{\left(\begin{array}{c}
2 k \\
k
\end{array}\right)}{16^{k}} T_{2 k}(2,3) \equiv \sum_{k=0}^{(p-1) / 2} \frac{\left(\begin{array}{c}
2 k \\
k
\end{array}\right)}{16^{k}} T_{2 k}(4,-3) \\
\equiv & \begin{cases}\left(\frac{-1}{p}\right)\left(\frac{x}{3}\right)\left(2 x-\frac{p}{2 x}\right)\left(\bmod p^{2}\right) & \text { if } p=x^{2}+3 y^{2}, \\
0(\bmod p) & \text { if } p \equiv 2(\bmod 3) .\end{cases}
\end{aligned}
$$

Also,

$$
\sum_{k=0}^{(p-1) / 2} \frac{\left(\begin{array}{c}
2 k \\
k
\end{array}\right)}{4^{k}} T_{2 k}(1,-3) \equiv \begin{cases}(-1)^{x y / 2}\left(\frac{x}{3}\right) 2 x(\bmod p) & \text { if } p=x^{2}+3 y^{2} \\
0(\bmod p) & \text { if } p \equiv 2(\bmod 3)\end{cases}
$$

and

$$
\begin{aligned}
& \sum_{k=0}^{(p-1) / 2} \frac{\left(\begin{array}{c}
2 k \\
k
\end{array}\right)}{16^{k}} T_{2 k}(4,3) \\
\equiv & \begin{cases}(-1)^{\lfloor x / 6\rfloor+y / 2} 2 x(\bmod p) & \text { if } 12 \mid p-1 \& p=x^{2}+y^{2}(2 \nmid x), \\
(-1)^{(x+y+1) / 2}\left(\frac{x y}{3}\right) 2 y(\bmod p) & \text { if } 12 \mid p-5 \& p=x^{2}+y^{2}(2 \nmid x), \\
0(\bmod p) & \text { if } p \equiv 3(\bmod 4) .\end{cases}
\end{aligned}
$$

Conjecture 2.3. Let $p$ be an odd prime. Then

$$
\begin{aligned}
& \sum_{k=0}^{(p-1) / 2} \frac{\left(\begin{array}{c}
2 k \\
k
\end{array}\right)}{16^{k}} T_{2 k}(12,-7) \\
\equiv & \begin{cases}2 x\left(\frac{x}{7}\right)(\bmod p) & \text { if }\left(\frac{p}{7}\right)=1 \& p=x^{2}+7 y^{2}, \\
0(\bmod p) & \text { if }\left(\frac{p}{7}\right)=-1, \text { i.e., } p \equiv 3,5,6(\bmod 7) .\end{cases}
\end{aligned}
$$

3. Extensions of (2)-(6) With APPLICATIONS TO SUMS INVOLVING GENERALIZED CENTRAL TRINOMIAL COEFFICIENTS

Our following theorem is a natural generalization of (2).

Theorem 3.1. For any $n \in \mathbb{N}$ we have

$$
D_{n}(x) D_{n}(y)=\sum_{k=0}^{n}\left(\begin{array}{c}
n+k \\
2 k
\end{array}\right)\left(\begin{array}{c}
2 k \\
k
\end{array}\right) \sum_{j=0}^{k}\left(\begin{array}{c}
k+j \\
2 j
\end{array}\right)\left(\begin{array}{c}
2 j \\
j
\end{array}\right)(x y+y)^{j}(x-y)^{k-j}
$$


Proof. Let $a_{n}$ denote the left hand side or the right-hand side of (17). It is easy to see that

$$
a_{0}=1, a_{1}=(2 x+1)(2 y+1), a_{2}=\left(6 x^{2}+6 x+1\right)\left(6 y^{2}+6 y+1\right)
$$

and

$$
a_{3}=\left(20 x^{3}+30 x^{2}+12 x+1\right)\left(20 y^{3}+30 y^{2}+12 y+1\right) .
$$

Applying the Zeilberger algorithm (cf. [PWZ, pp. 101-119]) via Mathematica we find the recursion for $n \geqslant 3$ :

$$
\begin{aligned}
& (n+1)^{2}(2 n-3) a_{n+1}-(2 n-3)(2 n+1)^{2}(2 x+1)(2 y+1) a_{n} \\
& +(2 n-1) A(n, x, y) a_{n-1}-(2 n-3)^{2}(2 n+1)(2 x+1)(2 y+1) a_{n-2} \\
& +(n-2)^{2}(2 n+1) a_{n-3} \\
& =0
\end{aligned}
$$

where

$$
A(n, x, y):=6 n^{2}-6 n-5+\left(16 n^{2}-16 n-12\right)\left(x+y-x^{2}-y^{2}\right) .
$$

Thus (17) holds by induction.

Now we give our extensions of $(3)-(6)$.

Theorem 3.2. Let $p$ be a prime and let $a \in \mathbb{Z}^{+}$. Let $h$ be a p-adic integer and set $w_{k}(h)=\left(\begin{array}{l}h \\ k\end{array}\right)\left(\begin{array}{c}h+k \\ k\end{array}\right)$ for $k \in \mathbb{N}$. Then

$$
\begin{aligned}
& \left(\sum_{k=0}^{p^{a}-1} w_{k}(h) x^{k}\right)\left(\sum_{k=0}^{p-1} w_{k}(h) y^{k}\right) \\
\equiv & \sum_{k=0}^{p^{a}-1} w_{k}(h) \sum_{j=0}^{k}\left(\begin{array}{c}
k+j \\
2 j
\end{array}\right)\left(\begin{array}{c}
2 j \\
j
\end{array}\right)(x y+y)^{j}(x-y)^{k-j}\left(\bmod p^{2}\right) .
\end{aligned}
$$

In particular, if $p \neq 2$ then

$$
\begin{aligned}
& \left(\sum_{k=0}^{p^{a}-1} \frac{\left(\begin{array}{c}
2 k \\
k
\end{array}\right)^{2}}{(-16)^{k}} x^{k}\right)\left(\sum_{k=0}^{p^{a}-1} \frac{\left(\begin{array}{c}
2 k \\
k
\end{array}\right)^{2}}{(-16)^{k}} y^{k}\right) \\
\equiv & \sum_{k=0}^{p^{a}-1} \frac{\left(\begin{array}{c}
2 k \\
k
\end{array}\right)^{2}}{(-16)^{k}} \sum_{j=0}^{k}\left(\begin{array}{c}
k+j \\
2 j
\end{array}\right)\left(\begin{array}{c}
2 j \\
j
\end{array}\right)(x y+y)^{j}(x-y)^{k-j}\left(\bmod p^{2}\right)
\end{aligned}
$$

and

$$
\begin{aligned}
& \left(\sum_{k=0}^{p^{a}-1} \frac{\left(\begin{array}{c}
4 k \\
2 k
\end{array}\right)\left(\begin{array}{c}
2 k \\
k
\end{array}\right)}{(-64)^{k}} x^{k}\right)\left(\sum_{k=0}^{p^{a}-1} \frac{\left(\begin{array}{c}
4 k \\
2 k
\end{array}\right)\left(\begin{array}{c}
2 k \\
k
\end{array}\right)}{(-64)^{k}} y^{k}\right) \\
\equiv & \sum_{k=0}^{p^{a}-1} \frac{\left(\begin{array}{c}
4 k \\
2 k
\end{array}\right)\left(\begin{array}{c}
2 k \\
k
\end{array}\right)}{(-64)^{k}} \sum_{j=0}^{k}\left(\begin{array}{c}
k+j \\
2 j
\end{array}\right)\left(\begin{array}{c}
2 j \\
j
\end{array}\right)(x y+y)^{j}(x-y)^{k-j}\left(\bmod p^{2}\right) ;
\end{aligned}
$$


provided $p>3$ we have

$$
\begin{aligned}
& \left(\sum_{k=0}^{p^{a}-1} \frac{\left(\begin{array}{c}
2 k \\
k
\end{array}\right)\left(\begin{array}{c}
3 k \\
k
\end{array}\right)}{(-27)^{k}} x^{k}\right)\left(\sum_{k=0}^{p^{a}-1} \frac{\left(\begin{array}{c}
2 k \\
k
\end{array}\right)\left(\begin{array}{c}
3 k \\
k
\end{array}\right)}{(-27)^{k}} y^{k}\right) \\
\equiv & \sum_{k=0}^{p^{a}-1} \frac{\left(\begin{array}{c}
2 k \\
k
\end{array}\right)\left(\begin{array}{c}
3 k \\
k
\end{array}\right)}{(-27)^{k}} \sum_{j=0}^{k}\left(\begin{array}{c}
k+j \\
2 j
\end{array}\right)\left(\begin{array}{c}
2 j \\
j
\end{array}\right)(x y+y)^{j}(x-y)^{k-j}\left(\bmod p^{2}\right)
\end{aligned}
$$

and

$$
\begin{aligned}
& \left(\sum_{k=0}^{p^{a}-1} \frac{\left(\begin{array}{c}
6 k \\
3 k
\end{array}\right)\left(\begin{array}{c}
3 k \\
k
\end{array}\right)}{(-432)^{k}} x^{k}\right)\left(\sum_{k=0}^{p^{a}-1} \frac{\left(\begin{array}{c}
6 k \\
3 k
\end{array}\right)\left(\begin{array}{c}
3 k \\
k
\end{array}\right)}{(-432)^{k}} y^{k}\right) \\
\equiv & \sum_{k=0}^{p^{a}-1} \frac{\left(\begin{array}{c}
6 k \\
3 k
\end{array}\right)\left(\begin{array}{c}
3 k \\
k
\end{array}\right)}{(-432)^{k}} \sum_{j=0}^{k}\left(\begin{array}{c}
k+j \\
2 j
\end{array}\right)\left(\begin{array}{c}
2 j \\
j
\end{array}\right)(x y+y)^{j}(x-y)^{k-j}\left(\bmod p^{2}\right) .
\end{aligned}
$$

Remark 3.1. Note that

$$
\begin{aligned}
& w_{k}\left(-\frac{1}{2}\right)=\frac{\left(\begin{array}{c}
2 k \\
k
\end{array}\right)^{2}}{(-16)^{k}}, \quad w_{k}\left(-\frac{1}{4}\right)=\frac{\left(\begin{array}{c}
4 k \\
2 k
\end{array}\right)\left(\begin{array}{c}
2 k \\
k
\end{array}\right)}{(-64)^{k}}, \\
& w_{k}\left(-\frac{1}{3}\right)=\frac{\left(\begin{array}{c}
2 k \\
k
\end{array}\right)\left(\begin{array}{c}
3 k \\
k
\end{array}\right)}{(-27)^{k}}, \quad w_{k}\left(-\frac{1}{6}\right)=\frac{\left(\begin{array}{c}
6 k \\
3 k
\end{array}\right)\left(\begin{array}{c}
3 k \\
k
\end{array}\right)}{(-432)^{k}} .
\end{aligned}
$$

Also, (19)-(22) in the case $x=y$ and $a=1$ yield (3)-(6) respectively.

The reader may wonder how we found Theorem 3.2. In fact, (17) is our main clue to the congruence (19). By refining our proof of (19)-(22) we found (18).

To prove Theorem 3.2 we need two lemmas.

Lemma 3.1. For $m, n \in \mathbb{N}$ we have

$$
\sum_{k=0}^{n}\left(\begin{array}{l}
n \\
k
\end{array}\right)\left(\begin{array}{c}
k+m \\
n
\end{array}\right) w_{k+m}(h)=\frac{w_{m}(h) w_{n}(h)}{\left(\begin{array}{c}
m+n \\
n
\end{array}\right)}
$$

where $w_{k}(h)=\left(\begin{array}{c}h \\ k\end{array}\right)\left(\begin{array}{c}h+k \\ k\end{array}\right)$ as defined in Theorem 3.2.

Proof. Let $u_{n}$ denote the left-hand side of (23). By applying the Zeilberger algorithm via Mathematica, we find the recursion:

$$
(n+1)(m+n+1) u_{n+1}=(h-n)(h+n+1) u_{n}(n=0,1,2, \ldots) .
$$

Thus (23) can be easily proved by induction on $n$. 
Lemma 3.2. For $k, m, n \in \mathbb{N}$ we have the combinatorial identity

$$
\begin{gathered}
\sum_{j=0}^{m}(-1)^{m-j}\left(\begin{array}{c}
m+j \\
2 j
\end{array}\right)\left(\begin{array}{c}
2 j \\
j
\end{array}\right)\left(\begin{array}{c}
j+k+m \\
k
\end{array}\right)\left(\begin{array}{l}
j \\
n
\end{array}\right) \\
=\left(\begin{array}{c}
k+m+n \\
m
\end{array}\right)\left(\begin{array}{c}
k+m \\
m
\end{array}\right)\left(\begin{array}{c}
m \\
n
\end{array}\right) .
\end{gathered}
$$

Proof. If $m<n$ then both sides of (24) vanish. (24) in the case $m=n$ can be directly verified. Let $s_{m}$ denote the left-hand side of (24). By the Zeilberger algorithm we find the recursion

$$
(m+1)(m-n+1) s_{m+1}=(k+m+1)(k+m+n+1) s_{m}(m=n, n+1, \ldots) .
$$

So we can show (24) by induction.

Proof of Theorem 3.2. In view of Remark 3.1, it suffices to prove (18). Note that both sides of (18) are polynomials in $x$ and $y$ and the degrees with respect to $x$ or $y$ are all smaller than $p^{a}$.

Fix $m, n \in\left\{0, \ldots, p^{a}-1\right\}$ and let $c(m, n)$ denote the coefficient of $x^{n} y^{m}$ in the right-hand side of (18). Define $\left(\begin{array}{c}z \\ -k\end{array}\right)=0$ for $k=1,2,3, \ldots$. Then $c(m, n)$ coincides with

$$
\begin{aligned}
& {\left[x^{n}\right] \sum_{0 \leqslant j \leqslant k<p^{a}} w_{k}(h)\left(\begin{array}{c}
k+j \\
2 j
\end{array}\right)\left(\begin{array}{c}
2 j \\
j
\end{array}\right)(x+1)^{j}\left(\begin{array}{c}
k-j \\
m-j
\end{array}\right)(-1)^{m-j} x^{k-m} } \\
= & \sum_{k=m}^{p^{a}-1} w_{k}(h) \sum_{j=0}^{m}(-1)^{m-j}\left(\begin{array}{c}
k+j \\
2 j
\end{array}\right)\left(\begin{array}{c}
2 j \\
j
\end{array}\right)\left(\begin{array}{c}
k-j \\
m-j
\end{array}\right)\left(\begin{array}{c}
j \\
m+n-k
\end{array}\right) \\
= & \sum_{k=0}^{p^{a}-1-m} w_{k+m}(h) \sum_{j=0}^{m}(-1)^{m-j}\left(\begin{array}{c}
k+m+j \\
2 j
\end{array}\right)\left(\begin{array}{c}
2 j \\
j
\end{array}\right)\left(\begin{array}{c}
k+m-j \\
k
\end{array}\right)\left(\begin{array}{c}
j \\
n-k
\end{array}\right) \\
= & \sum_{k=0}^{p^{a}-1-m} w_{k+m}(h) \sum_{j=0}^{m}(-1)^{m-j}\left(\begin{array}{c}
m+j \\
2 j
\end{array}\right)\left(\begin{array}{c}
2 j \\
j
\end{array}\right)\left(\begin{array}{c}
k+m+j \\
k
\end{array}\right)\left(\begin{array}{c}
j \\
n-k
\end{array}\right) .
\end{aligned}
$$

Applying Lemma 3.2 we get

$$
\begin{aligned}
c(m, n) & =\left(\begin{array}{c}
m+n \\
m
\end{array}\right) \sum_{k=0}^{p^{a}-1-m} w_{k+m}(h)\left(\begin{array}{c}
k+m \\
m
\end{array}\right)\left(\begin{array}{c}
m \\
n-k
\end{array}\right) \\
& =\left(\begin{array}{c}
m+n \\
m
\end{array}\right) \sum_{k=0}^{p^{a}-1-m} w_{k+m}(h)\left(\begin{array}{c}
k+m \\
n
\end{array}\right)\left(\begin{array}{l}
n \\
k
\end{array}\right) .
\end{aligned}
$$


By Lemma 3.1,

$$
\begin{aligned}
& \sum_{k=0}^{p^{a}-1} w_{k+m}(h)\left(\begin{array}{c}
k+m \\
n
\end{array}\right)\left(\begin{array}{l}
n \\
k
\end{array}\right) \\
= & \sum_{k=0}^{n} w_{k+m}(h)\left(\begin{array}{c}
k+m \\
n
\end{array}\right)\left(\begin{array}{l}
n \\
k
\end{array}\right)=\frac{w_{m}(h) w_{n}(h)}{\left(\begin{array}{c}
m+n \\
m
\end{array}\right)} .
\end{aligned}
$$

So, it remains to show

$$
\left(\begin{array}{c}
m+n \\
m
\end{array}\right) \sum_{k=p^{a}-m}^{p^{a}-1} w_{k+m}(h)\left(\begin{array}{c}
k+m \\
n
\end{array}\right)\left(\begin{array}{l}
n \\
k
\end{array}\right) \equiv 0\left(\bmod p^{2}\right) .
$$

To prove (25) we only need to show

$$
\left(\begin{array}{c}
m+n \\
m
\end{array}\right) \equiv\left(\begin{array}{c}
k+m \\
n
\end{array}\right) \equiv 0(\bmod p)
$$

under the supposition $n \geqslant k \geqslant p^{a}-m$. Note that $m+n \geqslant k+m \geqslant p^{a}$ and $0<p^{a}-n \leqslant k+m-n \leqslant m<p^{a}$. As the addition of $m$ and $n$ in base $p$ has at least one carry, we have $p \mid\left(\begin{array}{c}m+n \\ m\end{array}\right)$ by Kummer's theorem (cf. [Ri, p. 24]). Similarly, $p \mid\left(\begin{array}{c}k+m \\ n\end{array}\right)$.

So far we have completed the proof of Theorem 3.2.

Theorem 3.2 implies the following useful result on congruences for sums of central binomial coefficients and generalized central trinomial coefficients.

Theorem 3.3. Let $p$ be an odd prime and let $x$ be a p-adic integer. Let $a \in \mathbb{Z}^{+}, b, c \in \mathbb{Z}$ and $d=b^{2}-4 c$. Set $D:=1+2 b x+d x^{2}$. Then we have

$$
\begin{aligned}
& \sum_{k=0}^{p^{a}-1} \frac{\left(\begin{array}{c}
2 k \\
k
\end{array}\right)^{2}}{(-16)^{k}} T_{k}(b, c) x^{k} \\
\equiv & \left(\sum_{k=0}^{p^{a}-1} \frac{\left(\begin{array}{c}
2 k \\
k
\end{array}\right)^{2}}{32^{k}}(1-\sqrt{D}+\sqrt{d} x)^{k}\right)\left(\sum_{k=0}^{p^{a}-1} \frac{\left(\begin{array}{c}
2 k \\
k
\end{array}\right)^{2}}{32^{k}}(1-\sqrt{D}-\sqrt{d} x)^{k}\right) \\
\equiv & P_{\left(p^{a}-1\right) / 2}(\sqrt{D}+\sqrt{d} x) P_{\left(p^{a}-1\right) / 2}(\sqrt{D}-\sqrt{d} x)\left(\bmod p^{2}\right)
\end{aligned}
$$


and

$$
\begin{aligned}
\sum_{k=0}^{p^{a}-1} \frac{\left(\begin{array}{c}
4 k \\
2 k
\end{array}\right)\left(\begin{array}{c}
2 k \\
k
\end{array}\right)}{(-64)^{k}} T_{k}(b, c) x^{k} \equiv & \left(\sum_{k=0}^{p^{a}-1} \frac{\left(\begin{array}{c}
4 k \\
2 k
\end{array}\right)\left(\begin{array}{c}
2 k \\
k
\end{array}\right)}{128^{k}}(1-\sqrt{D}+\sqrt{d} x)^{k}\right) \\
& \times \sum_{k=0}^{p^{a}-1} \frac{\left(\begin{array}{c}
4 k \\
2 k
\end{array}\right)\left(\begin{array}{c}
2 k \\
k
\end{array}\right)}{128^{k}}(1-\sqrt{D}-\sqrt{d} x)^{k}\left(\bmod p^{2}\right) .
\end{aligned}
$$

If $p>3$, then

$$
\begin{aligned}
\sum_{k=0}^{p^{a}-1} \frac{\left(\begin{array}{c}
2 k \\
k
\end{array}\right)\left(\begin{array}{c}
3 k \\
k
\end{array}\right)}{(-27)^{k}} T_{k}(b, c) x^{k} \equiv & \left(\sum_{k=0}^{p^{a}-1} \frac{\left(\begin{array}{c}
2 k \\
k
\end{array}\right)\left(\begin{array}{c}
3 k \\
k
\end{array}\right)}{54^{k}}(1-\sqrt{D}+\sqrt{d} x)^{k}\right) \\
& \times \sum_{k=0}^{p^{a}-1} \frac{\left(\begin{array}{c}
2 k \\
k
\end{array}\right)\left(\begin{array}{c}
3 k \\
k
\end{array}\right)}{54^{k}}(1-\sqrt{D}-\sqrt{d} x)^{k}\left(\bmod p^{2}\right)
\end{aligned}
$$

and

$$
\begin{aligned}
\sum_{k=0}^{p^{a}-1} \frac{\left(\begin{array}{c}
6 k \\
3 k
\end{array}\right)\left(\begin{array}{c}
3 k \\
k
\end{array}\right)}{(-432)^{k}} T_{k}(b, c) x^{k} \equiv & \left(\sum_{k=0}^{p^{a}-1} \frac{\left(\begin{array}{c}
6 k \\
3 k
\end{array}\right)\left(\begin{array}{c}
3 k \\
k
\end{array}\right)}{864^{k}}(1-\sqrt{D}+\sqrt{d} x)^{k}\right) \\
& \times \sum_{k=0}^{p^{a}-1} \frac{\left(\begin{array}{c}
6 k \\
k
\end{array}\right)\left(\begin{array}{c}
3 k \\
k
\end{array}\right)}{864^{k}}(1-\sqrt{D}-\sqrt{d} x)^{k}\left(\bmod p^{2}\right) .
\end{aligned}
$$

Remark 3.2. Note that $\sqrt{d}$ and $\sqrt{D}$ in Theorem 3.3 are viewed as algebraic $p$-adic integers.

Proof of Theorem 3.3. Let $n=\left(p^{a}-1\right) / 2$. For $k=0, \ldots, n$ we have

$$
\left(\begin{array}{c}
n+k \\
2 k
\end{array}\right)=\frac{\left(\begin{array}{c}
2 k \\
k
\end{array}\right)}{(-16)^{k}} \prod_{0<j \leqslant k}\left(1-\frac{p^{2 a}}{(2 j-1)^{2}}\right) \equiv \frac{\left(\begin{array}{c}
2 k \\
k
\end{array}\right)}{(-16)^{k}}\left(\bmod p^{2}\right)
$$

and hence

$$
\left(\begin{array}{l}
n \\
k
\end{array}\right)\left(\begin{array}{c}
n+k \\
k
\end{array}\right)=\left(\begin{array}{c}
n+k \\
2 k
\end{array}\right)\left(\begin{array}{c}
2 k \\
k
\end{array}\right) \equiv \frac{\left(\begin{array}{c}
2 k \\
k
\end{array}\right)^{2}}{(-16)^{k}}\left(\bmod p^{2}\right)
$$


Note also that $p \mid\left(\begin{array}{c}2 k \\ k\end{array}\right)$ for $k=n+1, \ldots, p^{a}-1$ by Kummer's theorem. Thus

$$
\begin{aligned}
P_{n}(t) & =\sum_{k=0}^{n}\left(\begin{array}{c}
n \\
k
\end{array}\right)\left(\begin{array}{c}
n+k \\
k
\end{array}\right)\left(\frac{t-1}{2}\right)^{k} \\
& \equiv \sum_{k=0}^{n} \frac{\left(\begin{array}{c}
2 k \\
k
\end{array}\right)^{2}}{(-16)^{k}}\left(\frac{t-1}{2}\right)^{k} \equiv \sum_{k=0}^{p^{a}-1} \frac{\left(\begin{array}{c}
2 k \\
k
\end{array}\right)^{2}}{32^{k}}(1-t)^{k}\left(\bmod p^{2}\right),
\end{aligned}
$$

and hence the second congruence in (26) follows.

Set

Then

$$
u=\frac{\sqrt{D}+\sqrt{d} x-1}{2} \text { and } v=\frac{\sqrt{D}-\sqrt{d} x-1}{2} .
$$

$$
u v+v=\frac{D-(\sqrt{d} x+1)^{2}}{4}=\frac{b-\sqrt{d}}{2} x \quad \text { and } \quad u-v=\sqrt{d} x .
$$

In view of (10), for any $k \in \mathbb{N}$ we have

$$
\begin{aligned}
& \sum_{j=0}^{k}\left(\begin{array}{c}
k+j \\
2 j
\end{array}\right)\left(\begin{array}{c}
2 j \\
j
\end{array}\right)(u v+v)^{j}(u-v)^{k-j} \\
= & x^{k} \sum_{j=0}^{k}\left(\begin{array}{c}
k+j \\
2 j
\end{array}\right)\left(\begin{array}{c}
2 j \\
j
\end{array}\right)\left(\frac{b-\sqrt{d}}{2}\right)^{j} \sqrt{d}^{k-j}=x^{k} T_{k}(b, c) .
\end{aligned}
$$

So the first congruence in (26) follows from (19). Similarly, (27)-(29) are consequences of (20)-(22) respectively.

For $d \in\{2,3,4,7\}$, it is well known that an odd prime $p$ can be written in the form $x^{2}+d y^{2}$ with $x, y \in \mathbb{Z}$ if and only if $\left(\frac{-d}{p}\right)=1$ (see, e.g., $[\mathrm{BEW}]$ and $[\mathrm{Co}])$.

Applying (26) we get the following new results.

Theorem 3.4. Let $p$ be an odd prime. Then

$$
\sum_{k=0}^{p-1} \frac{\left(\begin{array}{c}
2 k \\
k
\end{array}\right)^{2} T_{k}(1,-2)}{32^{k}} \equiv \begin{cases}\left(\frac{2}{p}\right)\left(4 x^{2}-2 p\right)\left(\bmod p^{2}\right) & \text { if } p=x^{2}+4 y^{2} \\
0\left(\bmod p^{2}\right) & \text { if } p \equiv 3(\bmod 4) .\end{cases}
$$

Also,

$$
\begin{gathered}
\sum_{k=0}^{p-1} \frac{\left(\begin{array}{c}
2 k \\
k
\end{array}\right)^{2} T_{k}(2,-1)}{8^{k}} \equiv \begin{cases}\left(\frac{-1}{p}\right) 4 x^{2}(\bmod p) & \text { if } p=x^{2}+2 y^{2} \\
0\left(\bmod p^{2}\right) & \text { if } p \equiv 5,7(\bmod 8)\end{cases} \\
\sum_{k=0}^{p-1} \frac{\left(\begin{array}{c}
2 k \\
k
\end{array}\right)^{2} T_{k}(4,1)}{(-4)^{k}} \equiv \begin{cases}4 x^{2}(\bmod p) & \text { if } p=x^{2}+3 y^{2} \\
0\left(\bmod p^{2}\right) & \text { if } p \equiv 2(\bmod 3)\end{cases}
\end{gathered}
$$


and

$$
\begin{aligned}
& \sum_{k=0}^{p-1}(-1)^{k}\left(\begin{array}{c}
2 k \\
k
\end{array}\right)^{2} T_{k}(16,1) \equiv\left(\frac{-1}{p}\right) \sum_{k=0}^{p-1} \frac{\left(\begin{array}{c}
2 k \\
k
\end{array}\right)^{2} T_{k}(1,16)}{(-256)^{k}} \\
\equiv & \begin{cases}4 x^{2}(\bmod p) & \text { if }\left(\frac{p}{7}\right)=1 \text { and } p=x^{2}+7 y^{2}, \\
0\left(\bmod p^{2}\right) & \text { if }\left(\frac{p}{7}\right)=-1, \text { i.e., } p \equiv 3,5,6(\bmod 7) .\end{cases}
\end{aligned}
$$

Remark 3.3. Let $p$ be an odd prime. We guess that $4 x^{2}(\bmod p)$ in (31)-(33) can be replaced by $4 x^{2}-2 p\left(\bmod p^{2}\right)$.

To prove Theorem 3.4 we need a lemma.

Lemma 3.3. Let $p$ be an odd prime. Then

$$
\sum_{k=0}^{p-1} \frac{\left(\begin{array}{c}
2 k \\
k
\end{array}\right)^{2}}{32^{k}} x^{k} \equiv\left(\frac{2}{p}\right) x^{n} P_{n}\left(1-\frac{4}{x}\right)(\bmod p)
$$

and

$$
P_{n}(x) \equiv(2 x+2)^{n} P_{n}\left(\frac{3-x}{1+x}\right)(\bmod p)
$$

Proof. With the help of Lemma 2.1, we get

$$
\begin{aligned}
\sum_{k=0}^{p-1} \frac{\left(\begin{array}{c}
2 k \\
k
\end{array}\right)^{2}}{32^{k}} x^{k} & \equiv \sum_{k=0}^{n} \frac{\left(\begin{array}{c}
2 k \\
k
\end{array}\right)^{2}}{32^{k}} x^{k} \equiv \sum_{k=0}^{n} \frac{256^{k}\left(\begin{array}{c}
2(n-k) \\
n-k
\end{array}\right)^{2}}{32^{k}} x^{k}=\sum_{k=0}^{n}\left(\begin{array}{c}
2 k \\
k
\end{array}\right)^{2}(8 x)^{n-k} \\
& \equiv\left(\frac{8}{p}\right) x^{n} \sum_{k=0}^{n} \frac{\left(\begin{array}{c}
2 k \\
k
\end{array}\right)^{2}}{(-16)^{k}}\left(-\frac{2}{x}\right)^{k} \\
& \equiv\left(\frac{2}{p}\right) x^{n} \sum_{k=0}^{n}\left(\begin{array}{c}
n \\
k
\end{array}\right)\left(\begin{array}{c}
n+k \\
k
\end{array}\right)\left(\frac{(1-4 / x)-1}{2}\right)^{k} \\
& =\left(\frac{2}{p}\right) x^{n} P_{n}\left(1-\frac{4}{x}\right)(\bmod p) .
\end{aligned}
$$

This proves (34).

(35) follows from [S1, Theorem 2.6] and its proof.

Proof of Theorem 3.4. For convenience we set $n=(p-1) / 2$.

(i) Applying (26) with $b=1, c=-2$ and $x=-1 / 2$, we obtain that

$$
\sum_{k=0}^{p-1} \frac{\left(\begin{array}{c}
2 k \\
k
\end{array}\right)^{2}}{32^{k}} T_{k}(1,-2) \equiv\left(\sum_{k=0}^{p-1} \frac{\left(\begin{array}{c}
2 k \\
k
\end{array}\right)^{2}}{32^{k}}\right)\left(\sum_{k=0}^{p-1} \frac{\left(\begin{array}{c}
2 k \\
k
\end{array}\right)^{2}}{(-16)^{k}}\right)\left(\bmod p^{2}\right) .
$$


The author [Su2, Conjecture 5.5] conjectured that $\sum_{k=0}^{p-1}\left(\begin{array}{c}2 k \\ k\end{array}\right)^{2} / 32^{k} \equiv$ $0\left(\bmod p^{2}\right)$ if $p \equiv 3(\bmod 4)$, and

$$
\sum_{k=0}^{p-1} \frac{\left(\begin{array}{c}
2 k \\
k
\end{array}\right)^{2}}{32^{k}} \equiv\left(\frac{2}{p}\right) \sum_{k=0}^{p-1} \frac{\left(\begin{array}{c}
2 k \\
k
\end{array}\right)^{2}}{(-16)^{k}} \equiv 2 x-\frac{p}{2 x}\left(\bmod p^{2}\right)
$$

if $p=x^{2}+y^{2}$ with $x \equiv 1(\bmod 4)$ and $y \equiv 0(\bmod 2)$. This was confirmed by Z.-H. Sun [S1]. So the desired (30) follows.

(ii) Applying (26) with $b=2, c=-1$ and $x=-2$ we get

$$
\sum_{k=0}^{p-1} \frac{\left(\begin{array}{c}
2 k \\
k
\end{array}\right)^{2} T_{k}(2,-1)}{8^{k}} \equiv \sum_{k=0}^{n} \frac{\left(\begin{array}{c}
2 k \\
k
\end{array}\right)^{2}}{32^{k}} \alpha^{k} \times \sum_{k=0}^{n} \frac{\left(\begin{array}{c}
2 k \\
k
\end{array}\right)^{2}}{32^{k}} \beta^{k}\left(\bmod p^{2}\right) .
$$

where $\alpha=-4(1+\sqrt{2})$ and $\beta=-4(1-\sqrt{2})$. Clearly $\alpha \beta=-16$. By Lemma 3.3,

$$
\sum_{k=0}^{n} \frac{\left(\begin{array}{c}
2 k \\
k
\end{array}\right)^{2}}{32^{k}} \alpha^{k} \equiv\left(\frac{2}{p}\right) \alpha^{n} P_{n}(\sqrt{2})(\bmod p)
$$

and

$$
\sum_{k=0}^{n} \frac{\left(\begin{array}{c}
2 k \\
k
\end{array}\right)^{2}}{32^{k}} \beta^{k} \equiv\left(\frac{2}{p}\right) \beta^{n} P_{n}(-\sqrt{2})=\left(\frac{-2}{p}\right) \beta^{n} P_{n}(\sqrt{2})(\bmod p) .
$$

By [S2, Theorem 2.7], $P_{n}(\sqrt{2}) \equiv 0(\bmod p)$ if $\left(\frac{-2}{p}\right)=-1$, and $P_{n}(\sqrt{2})^{2} \equiv\left(\frac{-1}{p}\right) 4 x^{2}(\bmod p)$ if $\left(\frac{-2}{p}\right)=1$ and $p=x^{2}+2 y^{2}(x, y \in \mathbb{Z})$. So (31) holds.

(iii) (26) with $b=x=4$ and $c=1$ yields that

$$
\sum_{k=0}^{p-1} \frac{\left(\begin{array}{c}
2 k \\
k
\end{array}\right)^{2}}{(-4)^{k}} T_{k}(4,1) \equiv P_{n}(15+8 \sqrt{3}) P_{n}(15-8 \sqrt{3})\left(\bmod p^{2}\right) .
$$

By Lemma 3.3,

$$
\begin{aligned}
& ( \pm 1)^{n} P_{n}\left(\frac{\sqrt{3}}{2}\right)=P_{n}\left( \pm \frac{\sqrt{3}}{2}\right) \\
\equiv & (2 \pm \sqrt{3})^{n} P_{n}\left(\frac{3 \mp \sqrt{3} / 2}{1 \pm \sqrt{3} / 2}\right)=(2 \pm \sqrt{3})^{n} P_{n}(15 \mp 8 \sqrt{3})(\bmod p) .
\end{aligned}
$$

By [S2, Theorem 2.8], $P_{n}(\sqrt{3} / 2) \equiv 0(\bmod p)$ if $p \equiv 2(\bmod 3)$, and $P_{n}(\sqrt{3} / 2)^{2} \equiv(-1)^{n} 4 x^{2}(\bmod p)$ if $p \equiv 1(\bmod 3)$ and $p=$ $x^{2}+3 y^{2}(x, y \in \mathbb{Z})$. Therefore $(32)$ is valid. 
(iv) Applying (26) with $b=1, c=16$ and $x=1 / 16$ we obtain that

$$
\sum_{k=0}^{p-1} \frac{\left(\begin{array}{c}
2 k \\
k
\end{array}\right)^{2} T_{k}(1,16)}{(-256)^{k}} \equiv\left(\sum_{k=0}^{n} \frac{\left(\begin{array}{c}
2 k \\
k
\end{array}\right)^{2}}{32^{k}} \alpha^{k}\right) \times \sum_{k=0}^{n} \frac{\left(\begin{array}{c}
2 k \\
k
\end{array}\right)^{2}}{32^{k}} \beta^{k}\left(\bmod p^{2}\right)
$$

where $\alpha=(1+3 \sqrt{-7}) / 16$ and $\beta=(1-3 \sqrt{-7}) / 16$. Note that $\alpha \beta=1 / 4$. By Lemma 3.3,

$$
\sum_{k=0}^{p-1} \frac{\left(\begin{array}{c}
2 k \\
k
\end{array}\right)^{2}}{32^{k}} \alpha^{k} \equiv\left(\frac{2}{p}\right) \alpha^{n} P_{n}(\sqrt{-63})(\bmod p)
$$

and

$$
\sum_{k=0}^{p-1} \frac{\left(\begin{array}{c}
2 k \\
k
\end{array}\right)^{2}}{32^{k}} \beta^{k} \equiv\left(\frac{2}{p}\right) \beta^{n} P_{n}(-\sqrt{-63})=\left(\frac{-2}{p}\right) \beta^{n} P_{n}(\sqrt{-63})(\bmod p) .
$$

(26) with $b=16, c=1$ and $x=16$ yields that

$$
\sum_{k=0}^{p-1}(-1)^{k}\left(\begin{array}{c}
2 k \\
k
\end{array}\right)^{2} T_{k}(16,1) \equiv P_{n}(255+96 \sqrt{7}) P_{n}(255-96 \sqrt{7})\left(\bmod p^{2}\right) .
$$

By Lemma 3.3,

$$
( \pm 1)^{n} P_{n}\left(\frac{3 \sqrt{7}}{8}\right) \equiv(8 \pm 3 \sqrt{7})^{n} P_{n}(255 \mp 96 \sqrt{7})(\bmod p)
$$

Therefore

$$
\left(8^{2}-9 \times 7\right)^{n} \sum_{k=0}^{p-1}(-1)^{k}\left(\begin{array}{c}
2 k \\
k
\end{array}\right)^{2} T_{k}(16,1) \equiv(-1)^{n} P_{n}\left(\frac{3 \sqrt{7}}{8}\right)^{2}(\bmod p) .
$$

By [S2, Theorem 2.5], $P_{n}(\sqrt{-63}) \equiv P_{n}(3 \sqrt{7} / 8) \equiv 0(\bmod p)$ if $\left(\frac{p}{7}\right)=-1$, and

$$
P_{n}(\sqrt{-63})^{2} \equiv(-1)^{n} P_{n}\left(\frac{3 \sqrt{7}}{8}\right)^{2} \equiv 4 x^{2}(\bmod p)
$$

if $\left(\frac{p}{7}\right)=1$ and $p=x^{2}+7 y^{2}(x, y \in \mathbb{Z})$. Therefore (33) holds.

Motivated by Theorem 3.4 and the congruence

$$
\sum_{k=0}^{p-1}(21 k+8)\left(\begin{array}{c}
2 k \\
k
\end{array}\right)^{3} \equiv 8 p+16 p^{4} B_{p-3}\left(\bmod p^{5}\right)
$$


proved in [Su4] (where $B_{0}, B_{1}, \ldots$ are Bernoulli numbers), we conjecture that

$$
\begin{aligned}
\sum_{k=0}^{p-1}(3 k+1) \frac{\left(\begin{array}{c}
2 k \\
k
\end{array}\right)^{2} T_{k}(1,-2)}{32^{k}} & \equiv\left(\frac{-2}{p}\right) \frac{2 p}{3-\left(\frac{-1}{p}\right)}\left(\bmod p^{2}\right), \\
\sum_{k=0}^{p-1}(5 k+2) \frac{\left(\begin{array}{c}
2 k \\
k
\end{array}\right)^{2} T_{k}(2,-1)}{8^{k}} & \equiv p+p\left(\frac{-1}{p}\right)\left(\bmod p^{2}\right), \\
\sum_{k=0}^{p-1}(5 k+2) \frac{\left(\begin{array}{c}
2 k \\
k
\end{array}\right)^{2} T_{k}(4,1)}{(-4)^{k}} & \equiv \frac{2}{3} p\left(2\left(\frac{-1}{p}\right)+1\right)\left(\bmod p^{2}\right), \\
\sum_{k=0}^{p-1}(255 k+112)(-1)^{k}\left(\begin{array}{c}
2 k \\
k
\end{array}\right)^{2} T_{k}(16,1) & \equiv 16 p\left(3+4\left(\frac{-1}{p}\right)\right)\left(\bmod p^{2}\right), \\
\sum_{k=0}^{p-1}(30 k+7) \frac{\left(\begin{array}{c}
2 k \\
k
\end{array}\right)^{2} T_{k}(1,16)}{(-256)^{k}} & \equiv 7 p\left(\frac{-1}{p}\right)\left(\bmod p^{2}\right) .
\end{aligned}
$$

The last congruence led the author to find the conjectural identity

$$
\sum_{k=0}^{\infty} \frac{30 k+7}{(-256)^{k}}\left(\begin{array}{c}
2 k \\
k
\end{array}\right)^{2} T_{k}(1,16)=\frac{24}{\pi}
$$

in Jan. 2011 which was the starting point of the discovery of many series for $1 / \pi$ of new types given in Section 5 .

\section{Conjectural Congruences related to Representations \\ OF PRIMES BY BINARY QUADRATIC FORMS}

In view of (26), our following conjecture implies that for any prime $p=x^{2}+7 y^{2}$ with $x, y \in \mathbb{Z}^{+}$we have

$$
\sum_{k=0}^{p-1} \frac{\left(\begin{array}{c}
2 k \\
k
\end{array}\right)^{2} T_{k}(1,16)}{(-256)^{k}} \equiv\left(\frac{-1}{p}\right)\left(4 x^{2}-2 p\right)\left(\bmod p^{2}\right) .
$$

Conjecture 4.1. Let $p$ be an odd prime with $\left(\frac{p}{7}\right)=1$. Write $p=$ $x^{2}+7 y^{2}$ with $x, y \in \mathbb{Z}$ such that $x \equiv 1(\bmod 4)$ if $p \equiv 1(\bmod 4)$, and $y \equiv 1(\bmod 4)$ if $p \equiv 3(\bmod 4)$. Then

$$
\begin{aligned}
& \sum_{k=0}^{p-1} \frac{\left(\begin{array}{c}
2 k \\
k
\end{array}\right)^{2}}{256^{k}} u_{k}(1,16) \equiv \begin{cases}0\left(\bmod p^{2}\right) & \text { if } p \equiv 1(\bmod 4), \\
\frac{1}{3}\left(\frac{2}{p}\right)\left(\frac{p}{7 y}-4 y\right)\left(\bmod p^{2}\right) & \text { if } p \equiv 3(\bmod 4) ;\end{cases} \\
& \sum_{k=0}^{p-1} \frac{\left(\begin{array}{c}
2 k \\
k
\end{array}\right)^{2}}{256^{k}} v_{k}(1,16) \equiv \begin{cases}2\left(\frac{2}{p}\right)\left(2 x-\frac{p}{2 x}\right)\left(\bmod p^{2}\right) & \text { if } p \equiv 1(\bmod 4), \\
0\left(\bmod p^{2}\right) & \text { if } p \equiv 3(\bmod 4) .\end{cases}
\end{aligned}
$$


When $p \equiv 1(\bmod 4)$, we have

$$
\sum_{k=0}^{p-1} \frac{k\left(\begin{array}{c}
2 k \\
k
\end{array}\right)^{2}}{16^{k}} u_{k}(1,16) \equiv \sum_{k=0}^{p-1} \frac{k\left(\begin{array}{c}
2 k \\
k
\end{array}\right)^{2}}{256^{k}} u_{k}(1,16) \equiv \frac{\left(\frac{2}{p}\right)}{42}\left(x-\frac{p}{2 x}\right)\left(\bmod p^{2}\right)
$$

and

$$
\begin{aligned}
& \sum_{k=0}^{p-1}(4 k+3) \frac{\left(\begin{array}{c}
2 k \\
k
\end{array}\right)^{2}}{16^{k}} v_{k}(1,16) \\
\equiv & 3 \sum_{k=0}^{p-1}(4 k+1) \frac{\left(\begin{array}{c}
2 k \\
k
\end{array}\right)^{2}}{256^{k}} v_{k}(1,16) \equiv 6\left(\frac{2}{p}\right) x\left(\bmod p^{2}\right) .
\end{aligned}
$$

When $p \equiv 3(\bmod 4)$, we can determine $y \bmod p^{2}$ in the following way:

$$
\sum_{k=0}^{p-1} \frac{k\left(\begin{array}{c}
2 k \\
k
\end{array}\right)^{2}}{16^{k}} u_{k}(1,16) \equiv \sum_{k=0}^{p-1} \frac{k\left(\begin{array}{c}
2 k \\
k
\end{array}\right)^{2}}{16^{k}} v_{k}(1,16) \equiv-\left(\frac{2}{p}\right) \frac{y}{2}\left(\bmod p^{2}\right)
$$

and

$$
3 \sum_{k=0}^{p-1} \frac{k\left(\begin{array}{c}
2 k \\
k
\end{array}\right)^{2}}{256^{k}} u_{k}(1,16) \equiv \sum_{k=0}^{p-1} \frac{k\left(\begin{array}{c}
2 k \\
k
\end{array}\right)^{2}}{256^{k}} v_{k}(1,16) \equiv\left(\frac{2}{p}\right) \frac{y}{2}\left(\bmod p^{2}\right) .
$$

Just like $\mathbb{Q}(\sqrt{-7})$, the imaginary quadratic field $\mathbb{Q}(\sqrt{-11})$ also has class number one. Let $p$ be an odd prime. Whenever $\left(\frac{p}{11}\right)=1$ we can write $p$ in the form $\left(x^{2}+11 y^{2}\right) / 4$ with $x, y \in \mathbb{Z}$. We guess that

$$
\sum_{k=0}^{p-1} \frac{\left(\begin{array}{c}
2 k \\
k
\end{array}\right)\left(\begin{array}{c}
3 k \\
k
\end{array}\right) T_{k}(46,1)}{512^{k}} \equiv \begin{cases}x^{2}-2 p\left(\bmod p^{2}\right) & \text { if } 4 p=x^{2}+11 y^{2} \\
0\left(\bmod p^{2}\right) & \text { if }\left(\frac{p}{11}\right)=-1 .\end{cases}
$$

To attack this we note that (28) with $b=46, c=1$ and $x=-27 / 512$ yields

$$
\sum_{k=0}^{p-1} \frac{\left(\begin{array}{c}
2 k \\
k
\end{array}\right)\left(\begin{array}{c}
3 k \\
k
\end{array}\right)}{512^{k}} T_{k}(46,1) \equiv\left(\sum_{k=0}^{p-1} \frac{\left(\begin{array}{c}
2 k \\
k
\end{array}\right)\left(\begin{array}{c}
3 k \\
k
\end{array}\right)}{(-64)^{k}} \alpha^{k}\right) \times \sum_{k=0}^{p-1} \frac{\left(\begin{array}{c}
2 k \\
k
\end{array}\right)\left(\begin{array}{c}
3 k \\
k
\end{array}\right)}{(-64)^{k}} \beta^{k}\left(\bmod p^{2}\right),
$$

where $\alpha=(1+\sqrt{33}) / 2$ and $\beta=(1-\sqrt{33}) / 2$. Observe that $2 \alpha^{k}=$ $v_{k}(1,-8)+(\alpha-\beta) u_{k}(1,-8)$ and $2 \beta^{k}=v_{k}(1,-8)-(\alpha-\beta) u_{k}(1,-8)$. So we have

$$
\begin{aligned}
& 4 \sum_{k=0}^{p-1} \frac{\left(\begin{array}{c}
2 k \\
k
\end{array}\right)\left(\begin{array}{c}
3 k \\
k
\end{array}\right) T_{k}(46,1)}{512^{k}} \\
\equiv & \left(\sum_{k=0}^{p-1} \frac{\left(\begin{array}{c}
2 k \\
k
\end{array}\right)\left(\begin{array}{c}
3 k \\
k
\end{array}\right)}{(-64)^{k}} v_{k}(1,-8)\right)^{2}-33\left(\sum_{k=0}^{p-1} \frac{\left(\begin{array}{c}
2 k \\
k
\end{array}\right)\left(\begin{array}{c}
3 k \\
k
\end{array}\right)}{(-64)^{k}} v_{k}(1,-8)\right)^{2}\left(\bmod p^{2}\right) .
\end{aligned}
$$


This, together with the author's conjecture on $\sum_{k=0}^{p-1}\left(\begin{array}{c}2 k \\ k\end{array}\right)^{2}\left(\begin{array}{c}3 k \\ k\end{array}\right) / 64^{k} \bmod$ $p^{2}$ (cf. [Su2, Conjecture 5.4]) leads us to raise the following conjecture.

Conjecture 4.2. Let $p>3$ be a prime. If $\left(\frac{p}{11}\right)=-1$, then

$$
\sum_{k=0}^{p-1} \frac{\left(\begin{array}{c}
2 k \\
k
\end{array}\right)\left(\begin{array}{c}
3 k \\
k
\end{array}\right)}{(-64)^{k}} u_{k}(1,-8) \equiv \sum_{k=0}^{p-1} \frac{\left(\begin{array}{c}
2 k \\
k
\end{array}\right)\left(\begin{array}{c}
3 k \\
k
\end{array}\right)}{(-64)^{k}} v_{k}(1,-8) \equiv 0(\bmod p)
$$

When $\left(\frac{p}{11}\right)=1, p \equiv 1(\bmod 3)$, and $4 p=x^{2}+11 y^{2}$ with $x \equiv$ $1(\bmod 3)$, we have

$$
\begin{aligned}
\sum_{k=0}^{p-1} \frac{\left(\begin{array}{c}
2 k \\
k
\end{array}\right)\left(\begin{array}{c}
3 k \\
k
\end{array}\right)}{(-64)^{k}} u_{k}(1,-8) & \equiv 0\left(\bmod p^{2}\right), \\
\sum_{k=0}^{p-1} \frac{k\left(\begin{array}{c}
2 k \\
k
\end{array}\right)\left(\begin{array}{c}
3 k \\
k
\end{array}\right)}{(-64)^{k}} u_{k}(1,-8) & \equiv \frac{114}{11}\left(\frac{2 p}{x}-x\right)\left(\bmod p^{2}\right), \\
\sum_{k=0}^{p-1} \frac{k\left(\begin{array}{c}
2 k \\
k
\end{array}\right)\left(\begin{array}{c}
3 k \\
k
\end{array}\right)}{216^{k}} u_{k}(8,27) & \equiv \frac{4}{99}\left(\frac{2 p}{x}-x\right)\left(\bmod p^{2}\right), \\
\sum_{k=0}^{p-1} \frac{\left(\begin{array}{c}
2 k \\
k
\end{array}\right)\left(\begin{array}{c}
3 k \\
k
\end{array}\right)}{(-64)^{k}} v_{k}(1,-8) & \equiv \sum_{k=0}^{p-1} \frac{\left(\begin{array}{c}
2 k \\
k
\end{array}\right)\left(\begin{array}{c}
3 k \\
k
\end{array}\right)}{216^{k}} v_{k}(8,27) \equiv 2\left(\frac{p}{x}-x\right)\left(\bmod p^{2}\right),
\end{aligned}
$$

and

$$
\begin{aligned}
& \sum_{k=0}^{p-1}(k+60) \frac{\left(\begin{array}{c}
2 k \\
k
\end{array}\right)\left(\begin{array}{c}
3 k \\
k
\end{array}\right)}{(-64)^{k}} v_{k}(1,-8) \equiv-60 x\left(\bmod p^{2}\right), \\
& \sum_{k=0}^{p-1}(9 k+2) \frac{\left(\begin{array}{c}
2 k \\
k
\end{array}\right)\left(\begin{array}{c}
3 k \\
k
\end{array}\right)}{216^{k}} v_{k}(8,27) \equiv-2 x\left(\bmod p^{2}\right) .
\end{aligned}
$$

When $\left(\frac{p}{11}\right)=1, p \equiv 2(\bmod 3)$, and $4 p=x^{2}+11 y^{2}$ with $y \equiv$ $1(\bmod 3)$, we have

$$
\begin{aligned}
& 11 \sum_{k=0}^{p-1} \frac{\left(\begin{array}{c}
2 k \\
k
\end{array}\right)\left(\begin{array}{c}
3 k \\
k
\end{array}\right)}{(-64)^{k}} u_{k}(1,-8) \\
\equiv & -3 \sum_{k=0}^{p-1} \frac{\left(\begin{array}{c}
2 k \\
k
\end{array}\right)\left(\begin{array}{c}
3 k \\
k
\end{array}\right)}{(-64)^{k}} v_{k}(1,-8) \equiv \frac{3}{2}\left(\frac{p}{y}-11 y\right)\left(\bmod p^{2}\right),
\end{aligned}
$$




$$
\begin{gathered}
\sum_{k=0}^{p-1}(2 k-155) \frac{\left(\begin{array}{c}
2 k \\
k
\end{array}\right)\left(\begin{array}{c}
3 k \\
k
\end{array}\right)}{(-64)^{k}} u_{k}(1,-8) \equiv \frac{759}{2} y\left(\bmod p^{2}\right), \\
\sum_{k=0}^{p-1}(2 k-243) \frac{\left(\begin{array}{c}
2 k \\
k
\end{array}\right)\left(\begin{array}{c}
3 k \\
k
\end{array}\right)}{(-64)^{k}} v_{k}(1,-8) \equiv-\frac{4359}{2} y\left(\bmod p^{2}\right), \\
\sum_{k=0}^{p-1} \frac{\left(\begin{array}{c}
2 k \\
k
\end{array}\right)\left(\begin{array}{c}
3 k \\
k
\end{array}\right)}{216^{k}} u_{k}(8,27) \equiv y-\frac{p}{11 y}\left(\bmod p^{2}\right), \\
\sum_{k=0}^{p-1} \frac{k\left(\begin{array}{c}
2 k \\
k
\end{array}\right)\left(\begin{array}{c}
3 k \\
k
\end{array}\right)}{216^{k}} u_{k}(8,27) \equiv \frac{1}{8} \sum_{k=0}^{p-1} \frac{k\left(\begin{array}{c}
2 k \\
k
\end{array}\right)\left(\begin{array}{c}
3 k \\
k
\end{array}\right)}{216^{k}} v_{k}(8,27) \equiv-\frac{y}{9}\left(\bmod p^{2}\right) .
\end{gathered}
$$

Motivated by the author's investigation of $\sum_{k=0}^{p-1}\left(\begin{array}{c}2 k \\ k\end{array}\right)\left(\begin{array}{c}3 k \\ k\end{array}\right) T_{k}(3,1) / 27^{k}$ $\bmod p^{2}$ (with $p>3$ a prime) and the congruence (28), we pose the following conjecture which involves the well-known Fibonacci numbers $F_{k}=u_{k}(1,-1)(k \in \mathbb{N})$ and Lucas numbers $L_{k}=v_{k}(1,-1)(k \in \mathbb{N})$. Note that the imaginary quadratic field $\mathbb{Q}(\sqrt{-15})$ has class number 2 .

Conjecture 4.3. Let $p>5$ be a prime. If $p \equiv 1,4(\bmod 15)$ and $p=x^{2}+15 y^{2}(x, y \in \mathbb{Z})$ with $x \equiv 1(\bmod 3)$, then

$$
\begin{aligned}
\sum_{k=0}^{p-1} \frac{k\left(\begin{array}{c}
2 k \\
k
\end{array}\right)\left(\begin{array}{c}
3 k \\
k
\end{array}\right)}{27^{k}} F_{k} & \equiv \frac{2}{15}\left(\frac{p}{x}-2 x\right)\left(\bmod p^{2}\right), \\
\sum_{k=0}^{p-1} \frac{\left(\begin{array}{c}
2 k \\
k
\end{array}\right)\left(\begin{array}{c}
3 k \\
k
\end{array}\right)}{27^{k}} L_{k} & \equiv 4 x-\frac{p}{x}\left(\bmod p^{2}\right)
\end{aligned}
$$

and

$$
\sum_{k=0}^{p-1}(3 k+2) \frac{\left(\begin{array}{c}
2 k \\
k
\end{array}\right)\left(\begin{array}{c}
3 k \\
k
\end{array}\right)}{27^{k}} L_{k} \equiv 4 x\left(\bmod p^{2}\right) .
$$

If $p \equiv 2,8(\bmod 15)$ and $p=3 x^{2}+5 y^{2}(x, y \in \mathbb{Z})$ with $y \equiv 1(\bmod 3)$, then

$$
\sum_{k=0}^{p-1} \frac{\left(\begin{array}{c}
2 k \\
k
\end{array}\right)\left(\begin{array}{c}
3 k \\
k
\end{array}\right)}{27^{k}} F_{k} \equiv \frac{p}{5 y}-4 y\left(\bmod p^{2}\right)
$$

and

$$
\sum_{k=0}^{p-1} \frac{k\left(\begin{array}{c}
2 k \\
k
\end{array}\right)\left(\begin{array}{c}
3 k \\
k
\end{array}\right)}{27^{k}} F_{k} \equiv \sum_{k=0}^{p-1} \frac{k\left(\begin{array}{c}
2 k \\
k
\end{array}\right)\left(\begin{array}{c}
3 k \\
k
\end{array}\right)}{27^{k}} L_{k} \equiv \frac{4}{3} y\left(\bmod p^{2}\right) .
$$


Remark 4.1. By [Su8, Theorem 1.6], for any prime $p>3$ we have

$$
\sum_{k=0}^{p-1} \frac{\left(\begin{array}{c}
2 k \\
k
\end{array}\right)\left(\begin{array}{c}
3 k \\
k
\end{array}\right)}{27^{k}} F_{k} \equiv 0\left(\bmod p^{2}\right) \text { if } p \equiv 1(\bmod 3)
$$

and

$$
\sum_{k=0}^{p-1} \frac{\left(\begin{array}{c}
2 k \\
k
\end{array}\right)\left(\begin{array}{c}
3 k \\
k
\end{array}\right)}{27^{k}} L_{k} \equiv 0\left(\bmod p^{2}\right) \text { if } p \equiv 2(\bmod 3) .
$$

In fact, we have many other conjectures similar to Conjectures 4.14.3 ; for the sake of brevity we don't include them in this paper.

Conjecture 4.4. Let $p>3$ be a prime.

(i) If $p \equiv 1,4(\bmod 15)$ and $p=x^{2}+15 y^{2}$ with $x, y \in \mathbb{Z}$, then

$$
P_{(p-1) / 2}(7 \sqrt{-15} \pm 16 \sqrt{-3}) \equiv\left(\frac{-\sqrt{-15}}{p}\right)\left(\frac{x}{15}\right)\left(2 x-\frac{p}{2 x}\right)\left(\bmod p^{2}\right) .
$$

(ii) Suppose that $\left(\frac{p}{5}\right)=\left(\frac{p}{7}\right)=1$ and write $4 p=x^{2}+35 y^{2}$ with $x, y \in \mathbb{Z}$. If $p \equiv 1(\bmod 3)$, then

$$
\sum_{k=0}^{p-1} \frac{\left(\begin{array}{c}
2 k \\
k
\end{array}\right)\left(\begin{array}{c}
3 k \\
k
\end{array}\right)}{3456^{k}}(64+27 \sqrt{5} \pm \sqrt{-35})^{k} \equiv\left(\frac{x}{3}\right)\left(\frac{p}{x}-x\right)\left(\bmod p^{2}\right) .
$$

If $p \equiv 2(\bmod 3)$, then

$$
\sum_{k=0}^{p-1} \frac{\left(\begin{array}{c}
2 k \\
k
\end{array}\right)\left(\begin{array}{c}
3 k \\
k
\end{array}\right)}{3456^{k}}(64+27 \sqrt{5} \pm \sqrt{-35})^{k} \equiv \pm \sqrt{-35}\left(\frac{y}{3}\right)\left(y-\frac{p}{35 y}\right)\left(\bmod p^{2}\right) .
$$

(iii) If $\left(\frac{2}{p}\right)=\left(\frac{p}{3}\right)=\left(\frac{p}{5}\right)=1$ and $p=x^{2}+30 y^{2}$ with $x, y \in \mathbb{Z}$, then

$$
\sum_{k=0}^{p-1} \frac{\left(\begin{array}{c}
2 k \\
k
\end{array}\right)\left(\begin{array}{c}
3 k \\
k
\end{array}\right)}{2916^{k}}(54-35 \sqrt{2} \pm \sqrt{5})^{k} \equiv\left(\frac{x}{3}\right)\left(2 x-\frac{p}{2 x}\right)\left(\bmod p^{2}\right) .
$$

(iv) If $\left(\frac{-2}{p}\right)=\left(\frac{p}{3}\right)=\left(\frac{p}{7}\right)=1$, and $p=x^{2}+42 y^{2}$ with $x, y \in \mathbb{Z}$, then

$$
\sum_{k=0}^{p-1} \frac{\left(\begin{array}{c}
2 k \\
k
\end{array}\right)\left(\begin{array}{c}
3 k \\
k
\end{array}\right)}{13500^{k}}(250-99 \sqrt{6} \pm 2 \sqrt{14})^{k} \equiv\left(\frac{x}{3}\right)\left(2 x-\frac{p}{2 x}\right)\left(\bmod p^{2}\right) .
$$

(v) If $\left(\frac{2}{p}\right)=\left(\frac{p}{3}\right)=\left(\frac{p}{13}\right)=1$ and $p=x^{2}+78 y^{2}$ with $x, y \in \mathbb{Z}$, then

$$
\sum_{k=0}^{p-1} \frac{\left(\begin{array}{c}
2 k \\
k
\end{array}\right)\left(\begin{array}{c}
3 k \\
k
\end{array}\right)}{530604^{k}}(9826-6930 \sqrt{2} \pm 5 \sqrt{26})^{k} \equiv\left(\frac{x}{3}\right)\left(2 x-\frac{p}{2 x}\right)\left(\bmod p^{2}\right) \text {. }
$$


(vi) If $\left(\frac{2}{p}\right)=\left(\frac{p}{3}\right)=\left(\frac{p}{17}\right)=1$ and $p=x^{2}+102 y^{2}$ with $x, y \in \mathbb{Z}$, then $\sum_{k=0}^{p-1} \frac{\left(\begin{array}{c}2 k \\ k\end{array}\right)\left(\begin{array}{c}3 k \\ k\end{array}\right)}{3881196^{k}}(71874-17420 \sqrt{17} \pm 35 \sqrt{2})^{k} \equiv\left(\frac{x}{3}\right)\left(2 x-\frac{p}{2 x}\right)\left(\bmod p^{2}\right)$.

(vii) If $\left(\frac{-1}{p}\right)=\left(\frac{p}{3}\right)=\left(\frac{p}{11}\right)=1$ and $p=x^{2}+33 y^{2}$ with $x, y \in \mathbb{Z}$, then

$$
\sum_{k=0}^{p-1} \frac{\left(\begin{array}{c}
4 k \\
2 k
\end{array}\right)\left(\begin{array}{c}
2 k \\
k
\end{array}\right)}{\left(2^{12} 3\right)^{k}}(96-5 \sqrt{11} \pm 65 \sqrt{3})^{k} \equiv\left(\frac{x}{3}\right)\left(\frac{p}{2 x}-2 x\right)\left(\bmod p^{2}\right) .
$$

Remark 4.2. Let $p \equiv 1,4(\bmod 15)$ be a prime with $p=x^{2}+15 y^{2}$ $(x, y \in \mathbb{Z})$. Applying (26) we see that

$$
\begin{aligned}
& \sum_{k=0}^{p-1}(-1)^{k}\left(\begin{array}{c}
2 k \\
k
\end{array}\right)^{2} T_{k} \\
\equiv & P_{(p-1) / 2}(7 \sqrt{-15}+16 \sqrt{-3}) P_{(p-1) / 2}(7 \sqrt{-15}-16 \sqrt{-3})\left(\bmod p^{2}\right) .
\end{aligned}
$$

Thus part (i) of Conjecture 4.4 implies that

$$
\sum_{k=0}^{p-1}(-1)^{k}\left(\begin{array}{c}
2 k \\
k
\end{array}\right)^{2} T_{k} \equiv\left(2 x-\frac{p}{2 x}\right)^{2} \equiv 4 x^{2}-2 p\left(\bmod p^{2}\right) .
$$

We omit here similar comments on parts (ii)-(vii) of Conjecture 4.4. We also have many other conjectures similar to Conjecture 4.4.

Conjecture 4.5. Let $p>5$ be a prime. Then

$$
\begin{aligned}
& \left(\frac{-1}{p}\right) \sum_{k=0}^{p-1} \frac{\left(\begin{array}{c}
2 k \\
k
\end{array}\right)^{2} T_{2 k}(62,1)}{\left(-128^{2}\right)^{k}} \equiv\left(\frac{p}{3}\right) \sum_{k=0}^{p-1} \frac{\left(\begin{array}{c}
2 k \\
k
\end{array}\right)^{2} T_{2 k}(62,1)}{\left(-480^{2}\right)^{k}} \\
\equiv & \begin{cases}4 x^{2}-2 p\left(\bmod p^{2}\right) & \text { if } p \equiv 1,9(\bmod 20) \& p=x^{2}+5 y^{2}, \\
2 x^{2}-2 p\left(\bmod p^{2}\right) & \text { if } p \equiv 3,7(\bmod 20) \& 2 p=x^{2}+5 y^{2}, \\
0\left(\bmod p^{2}\right) & \text { if } p \equiv 11,13,17,19(\bmod 20) .\end{cases}
\end{aligned}
$$

And

$$
\begin{aligned}
& \sum_{k=0}^{p-1}(340 k+111) \frac{\left(\begin{array}{c}
2 k \\
k
\end{array}\right)^{2} T_{2 k}(62,1)}{\left(-128^{2}\right)^{k}} \equiv 3 p\left(\frac{-1}{p}\right)\left(22+15\left(\frac{p}{15}\right)\right)\left(\bmod p^{2}\right), \\
& \sum_{k=0}^{p-1}(340 k+59) \frac{\left(\begin{array}{c}
2 k \\
k
\end{array}\right)^{2} T_{2 k}(62,1)}{\left(-480^{2}\right)^{k}} \equiv p\left(\frac{-1}{p}\right)\left(51+8\left(\frac{p}{15}\right)\right)\left(\bmod p^{2}\right) .
\end{aligned}
$$


Conjecture 4.6. Let $p>3$ be a prime. Then

$$
\begin{aligned}
& \sum_{k=0}^{p-1} \frac{\left(\begin{array}{c}
2 k \\
k
\end{array}\right) T_{k}^{2}}{4^{k}} \equiv\left(\frac{p}{3}\right) \sum_{k=0}^{p-1} \frac{\left(\begin{array}{c}
2 k \\
k
\end{array}\right) T_{k}^{2}(4,1)}{16^{k}} \\
\equiv & \sum_{k=0}^{p-1} \frac{\left(\begin{array}{c}
2 k \\
k
\end{array}\right)^{2} T_{k}(10,1)}{(-64)^{k}} \equiv\left(\frac{p}{3}\right) \sum_{k=0}^{p-1} \frac{\left(\begin{array}{c}
2 k \\
k
\end{array}\right)^{2} T_{2 k}(6,1)}{256^{k}} \equiv \sum_{k=0}^{p-1} \frac{\left(\begin{array}{c}
2 k \\
k
\end{array}\right)^{2} T_{2 k}(6,1)}{1024^{k}} \\
\equiv & \begin{cases}4 x^{2}-2 p\left(\bmod p^{2}\right) & \text { if } p \equiv 1,7(\bmod 24), p=x^{2}+6 y^{2}, \\
8 x^{2}-2 p\left(\bmod p^{2}\right) & \text { if } p \equiv 5,11(\bmod 24), p=2 x^{2}+3 y^{2}, \\
0\left(\bmod p^{2}\right) & \text { if }\left(\frac{-6}{p}\right)=-1 ;\end{cases}
\end{aligned}
$$

and

$$
\begin{aligned}
& \sum_{k=0}^{p-1} \frac{\left(\begin{array}{c}
2 k \\
k
\end{array}\right) T_{k}^{2}(6,1)}{192^{k}} \\
\equiv & \begin{cases}\left(\frac{-1}{p}\right)\left(4 x^{2}-2 p\right)\left(\bmod p^{2}\right) & \text { if } p \equiv 1,7(\bmod 24), p=x^{2}+6 y^{2}, \\
8 x^{2}-2 p\left(\bmod p^{2}\right) & \text { if } p \equiv 5,11(\bmod 24), p=2 x^{2}+3 y^{2}, \\
0\left(\bmod p^{2}\right) & \text { if }\left(\frac{-6}{p}\right)=-1 .\end{cases}
\end{aligned}
$$

Also,

$$
\begin{aligned}
\sum_{k=0}^{p-1}(3 k+1) \frac{\left(\begin{array}{c}
2 k \\
k
\end{array}\right)^{2} T_{k}(10,1)}{(-64)^{k}} & \equiv \frac{p}{4}\left(3\left(\frac{p}{3}\right)+1\right)\left(\bmod p^{2}\right), \\
\sum_{k=0}^{p-1}(4 k+1) \frac{\left(\begin{array}{c}
2 k \\
k
\end{array}\right) T_{k}^{2}(6,1)}{192^{k}} & \equiv p\left(\frac{-6}{p}\right)\left(4-3\left(\frac{2}{p}\right)\right)\left(\bmod p^{2}\right) .
\end{aligned}
$$

Conjecture 4.7. Let $p>5$ be a prime.

(i) We have

$$
\begin{aligned}
& \sum_{k=0}^{p-1} \frac{\left(\begin{array}{c}
2 k \\
k
\end{array}\right) T_{k}^{2}(3,1)}{36^{k}} \equiv\left(\frac{2}{p}\right) \sum_{k=0}^{p-1} \frac{\left(\begin{array}{c}
2 k \\
k
\end{array}\right)^{2} T_{k}(34,1)}{(-64)^{k}} \equiv \sum_{k=0}^{p-1} \frac{\left(\begin{array}{c}
2 k \\
k
\end{array}\right)^{2} T_{2 k}(18,1)}{4096^{k}} \\
\equiv & \begin{cases}4 x^{2}-2 p\left(\bmod p^{2}\right) & \text { if } p \equiv 1,9,11,19(\bmod 40), p=x^{2}+10 y^{2}, \\
8 x^{2}-2 p\left(\bmod p^{2}\right) & \text { if } p \equiv 7,13,23,37(\bmod 40), p=2 x^{2}+5 y^{2}, \\
0\left(\bmod p^{2}\right) & \text { if }\left(\frac{-10}{p}\right)=-1 .\end{cases}
\end{aligned}
$$


Also,

$$
\begin{aligned}
\sum_{k=0}^{p-1}(16 k+5) \frac{\left(\begin{array}{c}
2 k \\
k
\end{array}\right) T_{k}(3,1)^{2}}{36^{k}} & \equiv 5 p\left(\bmod p^{2}\right), \\
\sum_{k=0}^{p-1}(60 k+23) \frac{\left(\begin{array}{c}
2 k \\
k
\end{array}\right)^{2} T_{k}(34,1)}{(-64)^{k}} & \equiv p\left(8\left(\frac{2}{p}\right)+15\left(\frac{-1}{p}\right)\right)\left(\bmod p^{2}\right) .
\end{aligned}
$$

Conjecture 4.8. Let $p>7$ be a prime. Then

$$
\begin{aligned}
& \sum_{k=0}^{p-1} \frac{\left(\begin{array}{c}
2 k \\
k
\end{array}\right)\left(\begin{array}{c}
3 k \\
k
\end{array}\right) T_{k}(18,1)}{512^{k}} \equiv\left(\frac{10}{p}\right) \sum_{k=0}^{p-1} \frac{\left(\begin{array}{c}
2 k \\
k
\end{array}\right)\left(\begin{array}{c}
3 k \\
k
\end{array}\right) T_{3 k}(6,1)}{(-512)^{k}} \\
\equiv & \begin{cases}x^{2}-2 p\left(\bmod p^{2}\right) & \text { if }\left(\frac{p}{5}\right)=\left(\frac{p}{7}\right)=1 \& 4 p=x^{2}+35 y^{2}, \\
2 p-5 x^{2}\left(\bmod p^{2}\right) & \text { if }\left(\frac{p}{5}\right)=\left(\frac{p}{7}\right)=-1 \& 4 p=5 x^{2}+7 y^{2}, \\
0\left(\bmod p^{2}\right) & \text { if }\left(\frac{p}{35}\right)=-1 .\end{cases}
\end{aligned}
$$

And

$$
\begin{aligned}
\sum_{k=0}^{p-1}(35 k+9) \frac{\left(\begin{array}{c}
2 k \\
k
\end{array}\right)\left(\begin{array}{c}
3 k \\
k
\end{array}\right) T_{k}(18,1)}{512^{k}} & \equiv \frac{9 p}{2}\left(7-5\left(\frac{p}{5}\right)\right)\left(\bmod p^{2}\right), \\
\sum_{k=0}^{p-1}(35 k+9) \frac{\left(\begin{array}{c}
2 k \\
k
\end{array}\right)^{2} T_{3 k}(6,1)}{(-512)^{k}} & \equiv \frac{9 p}{32}\left(\frac{2}{p}\right)\left(25+7\left(\frac{p}{7}\right)\right)\left(\bmod p^{2}\right) .
\end{aligned}
$$

Conjecture 4.9. Let $p \neq 2,29$ be a prime. When $p \neq 5,7$, we have

$$
\begin{aligned}
& \sum_{k=0}^{p-1} \frac{\left(\begin{array}{c}
2 k \\
k
\end{array}\right)^{2} T_{2 k}(19602,1)}{78400^{2 k}} \\
\equiv & \begin{cases}4 x^{2}-2 p\left(\bmod p^{2}\right) & \text { if }\left(\frac{-2}{p}\right)=\left(\frac{29}{p}\right)=1 \& p=x^{2}+58 y^{2}, \\
8 x^{2}-2 p\left(\bmod p^{2}\right) & \text { if }\left(\frac{-2}{p}\right)=\left(\frac{29}{p}\right)=-1 \& p=2 x^{2}+29 y^{2}, \\
0\left(\bmod p^{2}\right) & \text { if }\left(\frac{-58}{p}\right)=-1 .\end{cases}
\end{aligned}
$$

Provided $p \neq 13$ we have

$$
\begin{aligned}
& \sum_{k=0}^{p-1} \frac{\left(\begin{array}{c}
2 k \\
k
\end{array}\right)^{2} T_{2 k}(19602,1)}{78416^{2 k}} \\
\equiv & \begin{cases}4 x^{2}-2 p\left(\bmod p^{2}\right) & \text { if }\left(\frac{-2}{p}\right)=\left(\frac{29}{p}\right)=1 \& p=x^{2}+58 y^{2}, \\
2 p-8 x^{2}\left(\bmod p^{2}\right) & \text { if }\left(\frac{-2}{p}\right)=\left(\frac{29}{p}\right)=-1 \& p=2 x^{2}+29 y^{2}, \\
0\left(\bmod p^{2}\right) & \text { if }\left(\frac{-58}{p}\right)=-1 .\end{cases}
\end{aligned}
$$


Conjecture 4.10. Let $p>5$ be a prime. Then

$$
\begin{aligned}
& \left(\frac{-6}{p}\right) \sum_{k=0}^{p-1} \frac{\left(\begin{array}{c}
2 k \\
k
\end{array}\right)\left(\begin{array}{c}
3 k \\
k
\end{array}\right) T_{3 k}(26,1)}{(-24)^{3 k}} \equiv\left(\frac{15}{p}\right) \sum_{k=0}^{p-1} \frac{\left(\begin{array}{c}
2 k \\
k
\end{array}\right)\left(\begin{array}{c}
3 k \\
k
\end{array}\right) T_{3 k}(62,1)}{(-240)^{3 k}} \\
\equiv & \begin{cases}x^{2}-2 p\left(\bmod p^{2}\right) & \text { if }\left(\frac{p}{7}\right)=\left(\frac{p}{13}\right)=1 \& 4 p=x^{2}+91 y^{2}, \\
2 p-7 x^{2}\left(\bmod p^{2}\right) & \text { if }\left(\frac{p}{7}\right)=\left(\frac{p}{13}\right)=-1 \& 4 p=7 x^{2}+13 y^{2}, \\
0\left(\bmod p^{2}\right) & \text { if }\left(\frac{p}{91}\right)=-1 .\end{cases}
\end{aligned}
$$

And

$$
\begin{aligned}
& \sum_{k=0}^{p-1}(819 k+239) \frac{\left(\begin{array}{c}
2 k \\
k
\end{array}\right)\left(\begin{array}{c}
3 k \\
k
\end{array}\right) T_{3 k}(26,1)}{(-24)^{3 k}} \\
\equiv & \frac{p}{32}\left(\frac{-6}{p}\right)\left(949+6699\left(\frac{p}{7}\right)\right)\left(\bmod p^{2}\right), \\
& \sum_{k=0}^{p-1}(1638 k+277) \frac{\left(\begin{array}{c}
2 k \\
k
\end{array}\right)\left(\begin{array}{c}
3 k \\
k
\end{array}\right) T_{3 k}(62,1)}{(-240)^{3 k}} \\
\equiv & \frac{p}{40}\left(\frac{-105}{p}\right)\left(8701+2379\left(\frac{p}{7}\right)\right)\left(\bmod p^{2}\right) .
\end{aligned}
$$

Remark 4.3. Note that the imaginary quadratic field $\mathbb{Q}(\sqrt{-d})$ has class number two for $d=5,6,10,15,35,58,91$.

Conjecture 4.11. Let $p>3$ be a prime. We have

$$
\begin{aligned}
& \left(\frac{-6}{p}\right) \sum_{k=0}^{p-1} \frac{\left(\begin{array}{c}
4 k \\
2 k
\end{array}\right)\left(\begin{array}{c}
2 k \\
k
\end{array}\right) T_{k}(110,1)}{\left(-96^{2}\right)^{k}} \\
& \equiv \begin{cases}4 x^{2}-2 p\left(\bmod p^{2}\right) & \text { if }\left(\frac{-1}{p}\right)=\left(\frac{p}{3}\right)=\left(\frac{p}{7}\right)=1, p=x^{2}+21 y^{2}, \\
12 x^{2}-2 p\left(\bmod p^{2}\right) & \text { if }\left(\frac{-1}{p}\right)=\left(\frac{p}{7}\right)=-1,\left(\frac{p}{3}\right)=1, p=3 x^{2}+7 y^{2}, \\
2 x^{2}-2 p\left(\bmod p^{2}\right) & \text { if }\left(\frac{-1}{p}\right)=\left(\frac{p}{3}\right)=-1,\left(\frac{p}{7}\right)=1,2 p=x^{2}+21 y^{2}, \\
6 x^{2}-2 p\left(\bmod p^{2}\right) & \text { if }\left(\frac{-1}{p}\right)=1,\left(\frac{p}{3}\right)=\left(\frac{p}{7}\right)=-1,2 p=3 x^{2}+7 y^{2}, \\
0\left(\bmod p^{2}\right) & \text { if }\left(\frac{-21}{p}\right)=-1,\end{cases}
\end{aligned}
$$

and

$$
\sum_{k=0}^{p-1}(28 k+5) \frac{\left(\begin{array}{c}
4 k \\
2 k
\end{array}\right)\left(\begin{array}{c}
2 k \\
k
\end{array}\right) T_{k}(110,1)}{\left(-96^{2}\right)^{k}} \equiv \frac{p}{8}\left(\frac{-6}{p}\right)\left(33+7\left(\frac{p}{7}\right)\right)\left(\bmod p^{2}\right) .
$$


Conjecture 4.12. Let $p>3$ be a prime. Then

$$
\begin{aligned}
& \sum_{k=0}^{p-1} \frac{\left(\begin{array}{c}
2 k \\
k
\end{array}\right)^{2} T_{2 k}(18,1)}{256^{k}} \\
& \equiv \begin{cases}4 x^{2}-2 p\left(\bmod p^{2}\right) & \text { if }\left(\frac{2}{p}\right)=\left(\frac{p}{3}\right)=\left(\frac{p}{5}\right)=1, p=x^{2}+30 y^{2}, \\
12 x^{2}-2 p\left(\bmod p^{2}\right) & \text { if }\left(\frac{p}{3}\right)=1,\left(\frac{2}{p}\right)=\left(\frac{p}{5}\right)=-1, p=3 x^{2}+10 y^{2}, \\
2 p-8 x^{2}\left(\bmod p^{2}\right) & \text { if }\left(\frac{2}{p}\right)=1,\left(\frac{p}{3}\right)=\left(\frac{p}{5}\right)=-1, p=2 x^{2}+15 y^{2}, \\
2 p-6 x^{2}\left(\bmod p^{2}\right) & \text { if }\left(\frac{p}{5}\right)=1,\left(\frac{2}{p}\right)=\left(\frac{p}{3}\right)=-1,2 p=3 x^{2}+10 y^{2}, \\
0\left(\bmod p^{2}\right) & \text { if }\left(\frac{-30}{p}\right)=-1 .\end{cases}
\end{aligned}
$$

And

$$
\begin{aligned}
& \sum_{k=0}^{p-1} \frac{\left(\begin{array}{c}
2 k \\
k
\end{array}\right)^{2} T_{2 k}(30,1)}{256^{k}} \\
& \equiv \begin{cases}4 x^{2}-2 p\left(\bmod p^{2}\right) & \text { if }\left(\frac{-2}{p}\right)=\left(\frac{p}{3}\right)=\left(\frac{p}{7}\right)=1, p=x^{2}+42 y^{2}, \\
12 x^{2}-2 p\left(\bmod p^{2}\right) & \text { if }\left(\frac{-2}{p}\right)=1,\left(\frac{p}{3}\right)=\left(\frac{p}{7}\right)=-1, p=3 x^{2}+14 y^{2}, \\
2 p-8 x^{2}\left(\bmod p^{2}\right) & \text { if }\left(\frac{p}{7}\right)=1,\left(\frac{-2}{p}\right)=\left(\frac{p}{3}\right)=-1, p=2 x^{2}+21 y^{2}, \\
2 p-6 x^{2}\left(\bmod p^{2}\right) & \text { if }\left(\frac{p}{3}\right)=1,\left(\frac{-2}{p}\right)=\left(\frac{p}{7}\right)=-1,2 p=3 x^{2}+14 y^{2}, \\
0\left(\bmod p^{2}\right) & \text { if }\left(\frac{-42}{p}\right)=-1 .\end{cases}
\end{aligned}
$$

Conjecture 4.13. Let $p>3$ be a prime. When $p \neq 13,17$, we have

$$
\begin{aligned}
& \sum_{k=0}^{p-1} \frac{\left(\begin{array}{c}
2 k \\
k
\end{array}\right)\left(\begin{array}{c}
3 k \\
k
\end{array}\right) T_{k}(102,1)}{102^{3 k}} \\
& \equiv \begin{cases}4 x^{2}-2 p\left(\bmod p^{2}\right) & \text { if }\left(\frac{2}{p}\right)=\left(\frac{p}{3}\right)=\left(\frac{p}{13}\right)=1, p=x^{2}+78 y^{2}, \\
2 p-8 x^{2}\left(\bmod p^{2}\right) & \text { if }\left(\frac{2}{p}\right)=1,\left(\frac{p}{3}\right)=\left(\frac{p}{13}\right)=-1, p=2 x^{2}+39 y^{2}, \\
12 x^{2}-2 p\left(\bmod p^{2}\right) & \text { if }\left(\frac{p}{13}\right)=1,\left(\frac{2}{p}\right)=\left(\frac{p}{3}\right)=-1, p=3 x^{2}+26 y^{2}, \\
2 p-24 x^{2}\left(\bmod p^{2}\right) & \text { if }\left(\frac{p}{3}\right)=1,\left(\frac{2}{p}\right)=\left(\frac{p}{13}\right)=-1, p=6 x^{2}+13 y^{2}, \\
0\left(\bmod p^{2}\right) & \text { if }\left(\frac{-78}{p}\right)=-1 .\end{cases}
\end{aligned}
$$


Provided $p \neq 11,17$, we have

$$
\begin{aligned}
& \sum_{k=0}^{p-1} \frac{\left(\begin{array}{c}
2 k \\
k
\end{array}\right)\left(\begin{array}{c}
3 k \\
k
\end{array}\right) T_{k}(198,1)}{198^{3 k}} \\
& \equiv \begin{cases}4 x^{2}-2 p\left(\bmod p^{2}\right) & \text { if }\left(\frac{2}{p}\right)=\left(\frac{p}{3}\right)=\left(\frac{p}{17}\right)=1, p=x^{2}+102 y^{2}, \\
2 p-8 x^{2}\left(\bmod p^{2}\right) & \text { if }\left(\frac{p}{17}\right)=1,\left(\frac{2}{p}\right)=\left(\frac{p}{3}\right)=-1, p=2 x^{2}+51 y^{2}, \\
12 x^{2}-2 p\left(\bmod p^{2}\right) & \text { if }\left(\frac{p}{3}\right)=1,\left(\frac{2}{p}\right)=\left(\frac{p}{17}\right)=-1, p=3 x^{2}+34 y^{2}, \\
2 p-24 x^{2}\left(\bmod p^{2}\right) & \text { if }\left(\frac{2}{p}\right)=1,\left(\frac{p}{3}\right)=\left(\frac{p}{17}\right)=-1, p=6 x^{2}+17 y^{2}, \\
0\left(\bmod p^{2}\right) & \text { if }\left(\frac{-102}{p}\right)=-1 .\end{cases}
\end{aligned}
$$

Conjecture 4.14. Let $p$ be an odd prime and let $m$ belong to the set $\{2,3,6,10,18,30,102,198\}$. If $p \nmid m$, then

$$
\sum_{k=0}^{p-1} \frac{\left(\begin{array}{c}
2 k \\
k
\end{array}\right)\left(\begin{array}{c}
3 k \\
k
\end{array}\right) T_{k}(m, 1)}{m^{3 k}} \equiv \sum_{k=0}^{p-1} \frac{\left(\begin{array}{c}
2 k \\
k
\end{array}\right)^{2} T_{2 k}(m, 1)}{256^{k}}\left(\bmod p^{2}\right) .
$$

If $m^{2} \not \equiv-12(\bmod p)$, then

$$
\sum_{k=0}^{p-1} \frac{\left(\begin{array}{c}
2 k \\
k
\end{array}\right)^{2} T_{2 k}(m, 1)}{256^{k}} \equiv\left(\frac{m^{2}+12}{p}\right) \sum_{k=0}^{p-1} \frac{\left(\begin{array}{c}
4 k \\
2 k
\end{array}\right)\left(\begin{array}{c}
2 k \\
k
\end{array}\right) T_{k}\left(m^{2}-2,1\right)}{\left(m^{2}+12\right)^{2 k}}\left(\bmod p^{2}\right) .
$$

Remark 4.4. We note that (36) holds mod $p$ for any integer $m \not \equiv$ $0(\bmod p)$, and $(37)$ holds $\bmod p$ for any $m \in \mathbb{Z}$ with $m^{2} \not \equiv-12(\bmod p)$.

Conjecture 4.15. Let $p \neq 2,5,19$ be a prime. We have

$$
\begin{aligned}
& \sum_{k=0}^{p-1} \frac{\left(\begin{array}{c}
2 k \\
k
\end{array}\right)^{2} T_{2 k}(5778,1)}{1216^{2 k}} \\
\equiv & \begin{cases}4 x^{2}-2 p\left(\bmod p^{2}\right) & \text { if }\left(\frac{2}{p}\right)=\left(\frac{p}{5}\right)=\left(\frac{p}{19}\right)=1, p=x^{2}+190 y^{2}, \\
8 x^{2}-2 p\left(\bmod p^{2}\right) & \text { if }\left(\frac{2}{p}\right)=1,\left(\frac{p}{5}\right)=\left(\frac{p}{19}\right)=-1, p=2 x^{2}+95 y^{2}, \\
2 p-20 x^{2}\left(\bmod p^{2}\right) & \text { if }\left(\frac{2}{p}\right)=\left(\frac{p}{5}\right)=-1,\left(\frac{p}{19}\right)=1, p=5 x^{2}+38 y^{2}, \\
2 p-40 x^{2}\left(\bmod p^{2}\right) & \text { if }\left(\frac{2}{p}\right)=\left(\frac{p}{19}\right)=-1,\left(\frac{p}{5}\right)=1, p=10 x^{2}+19 y^{2}, \\
0\left(\bmod p^{2}\right) & \text { if }\left(\frac{-190}{p}\right)=-1,\end{cases}
\end{aligned}
$$

and

$$
\begin{aligned}
& \sum_{k=0}^{p-1}(57720 k+24893) \frac{\left(\begin{array}{c}
2 k \\
k
\end{array}\right)^{2} T_{2 k}(5778,1)}{1216^{2 k}} \\
& \equiv p\left(11548+13345\left(\frac{p}{95}\right)\right)\left(\bmod p^{2}\right)
\end{aligned}
$$


Provided $p \neq 17$ we have

$$
\begin{gathered}
\sum_{k=0}^{p-1} \frac{\left(\begin{array}{c}
2 k \\
k
\end{array}\right)^{2} T_{2 k}(5778,1)}{439280^{2 k}} \equiv\left(\frac{p}{5}\right) \sum_{k=0}^{p-1} \frac{\left(\begin{array}{c}
2 k \\
k
\end{array}\right)^{2} T_{2 k}(5778,1)}{1216^{2 k}}\left(\bmod p^{2}\right) \\
\sum_{k=0}^{p-1}(57720 k+3967) \frac{\left(\begin{array}{c}
2 k \\
k
\end{array}\right)^{2} T_{2 k}(5778,1)}{439280^{2 k}} \\
\equiv p\left(\frac{p}{19}\right)\left(3983-16\left(\frac{p}{95}\right)\right)\left(\bmod p^{2}\right) .
\end{gathered}
$$

Conjecture 4.16. Let $p>5$ be a prime. Then

$$
\begin{aligned}
& \sum_{k=0}^{p-1} \frac{\left(\begin{array}{c}
2 k \\
k
\end{array}\right)^{2} T_{2 k}(198,1)}{224^{2 k}} \equiv\left(\frac{p}{7}\right) \sum_{k=0}^{p-1} \frac{\left(\begin{array}{c}
2 k \\
k
\end{array}\right)^{2} T_{2 k}(322,1)}{48^{4 k}} \\
& \equiv \begin{cases}4 x^{2}-2 p\left(\bmod p^{2}\right) & \text { if }\left(\frac{2}{p}\right)=\left(\frac{p}{5}\right)=\left(\frac{p}{7}\right)=1, p=x^{2}+70 y^{2}, \\
8 x^{2}-2 p\left(\bmod p^{2}\right) & \text { if }\left(\frac{p}{7}\right)=1,\left(\frac{2}{p}\right)=\left(\frac{p}{5}\right)=-1, p=2 x^{2}+35 y^{2}, \\
2 p-20 x^{2}\left(\bmod p^{2}\right) & \text { if }\left(\frac{p}{5}\right)=1,\left(\frac{2}{p}\right)=\left(\frac{p}{7}\right)=-1, p=5 x^{2}+14 y^{2}, \\
28 x^{2}-2 p\left(\bmod p^{2}\right) & \text { if }\left(\frac{2}{p}\right)=1,\left(\frac{p}{5}\right)=\left(\frac{p}{7}\right)=-1, p=7 x^{2}+10 y^{2}, \\
0\left(\bmod p^{2}\right) & \text { if }\left(\frac{-70}{p}\right)=-1 .\end{cases}
\end{aligned}
$$

Also,

$$
\begin{aligned}
& \sum_{k=0}^{p-1} \frac{\left(\begin{array}{c}
2 k \\
k
\end{array}\right)^{2} T_{2 k}(322,1)}{\left(-2^{10} 3^{4}\right)^{k}} \\
& \equiv \begin{cases}4 x^{2}-2 p\left(\bmod p^{2}\right) & \text { if }\left(\frac{-1}{p}\right)=\left(\frac{p}{5}\right)=\left(\frac{p}{17}\right)=1, p=x^{2}+85 y^{2}, \\
2 p-2 x^{2}\left(\bmod p^{2}\right) & \text { if }\left(\frac{p}{17}\right)=1,\left(\frac{-1}{p}\right)=\left(\frac{p}{5}\right)=-1,2 p=x^{2}+85 y^{2}, \\
2 p-20 x^{2}\left(\bmod p^{2}\right) & \text { if }\left(\frac{-1}{p}\right)=1,\left(\frac{p}{5}\right)=\left(\frac{p}{17}\right)=-1, p=5 x^{2}+17 y^{2}, \\
10 x^{2}-2 p\left(\bmod p^{2}\right) & \text { if }\left(\frac{p}{5}\right)=1,\left(\frac{-1}{p}\right)=\left(\frac{p}{17}\right)=-1,2 p=5 x^{2}+17 y^{2}, \\
0\left(\bmod p^{2}\right) & \text { if }\left(\frac{-85}{p}\right)=-1 .\end{cases}
\end{aligned}
$$

And

$$
\begin{aligned}
& \sum_{k=0}^{p-1} \frac{\left(\begin{array}{c}
2 k \\
k
\end{array}\right)^{2} T_{2 k}(1298,1)}{24^{4 k}} \\
& \equiv \begin{cases}4 x^{2}-2 p\left(\bmod p^{2}\right) & \text { if }\left(\frac{-2}{p}\right)=\left(\frac{p}{5}\right)=\left(\frac{p}{13}\right)=1, p=x^{2}+130 y^{2}, \\
8 x^{2}-2 p\left(\bmod p^{2}\right) & \text { if }\left(\frac{-2}{p}\right)=1,\left(\frac{p}{5}\right)=\left(\frac{p}{13}\right)=-1, p=2 x^{2}+65 y^{2}, \\
2 p-20 x^{2}\left(\bmod p^{2}\right) & \text { if }\left(\frac{p}{5}\right)=1,\left(\frac{-2}{p}\right)=\left(\frac{p}{13}\right)=-1, p=5 x^{2}+26 y^{2}, \\
2 p-40 x^{2}\left(\bmod p^{2}\right) & \text { if }\left(\frac{p}{13}\right)=1,\left(\frac{-2}{p}\right)=\left(\frac{p}{5}\right)=-1, p=10 x^{2}+13 y^{2}, \\
0\left(\bmod p^{2}\right) & \text { if }\left(\frac{-130}{p}\right)=-1 .\end{cases}
\end{aligned}
$$


Remark 4.5. . The imaginary quadratic field $\mathbb{Q}(\sqrt{-d})$ has class number four for $d=21,30,42,70,78,85,102,130,190$.

Conjecture 4.17. Let $p>3$ be a prime. Then

$$
\begin{aligned}
& \sum_{k=0}^{p-1} \frac{\left(\begin{array}{c}
2 k \\
k
\end{array}\right)\left(\begin{array}{c}
3 k \\
k
\end{array}\right) T_{3 k}}{(-27)^{k}} \equiv \begin{cases}\left(\frac{p}{3}\right)\left(4 x^{2}-2 p\right)\left(\bmod p^{2}\right) & \text { if } p=x^{2}+7 y^{2} \\
0\left(\bmod p^{2}\right) & \text { if }\left(\frac{p}{7}\right)=-1\end{cases} \\
& \sum_{k=0}^{p-1} \frac{\left(\begin{array}{c}
2 k \\
k
\end{array}\right)\left(\begin{array}{c}
3 k \\
k
\end{array}\right) T_{3 k}(26,81)}{24^{3 k}} \equiv \begin{cases}\left(\frac{6}{p}\right)\left(x^{2}-2 p\right)\left(\bmod p^{2}\right) & \text { if } 4 p=x^{2}+11 y^{2} \\
0\left(\bmod p^{2}\right) & \text { if }\left(\frac{p}{11}\right)=-1\end{cases} \\
& \sum_{k=0}^{p-1} \frac{\left(\begin{array}{c}
2 k \\
k
\end{array}\right)\left(\begin{array}{c}
3 k \\
k
\end{array}\right) T_{3 k}(10,1)}{24^{3 k}} \equiv \begin{cases}\left(\frac{6}{p}\right)\left(x^{2}-2 p\right)\left(\bmod p^{2}\right) & \text { if } 4 p=x^{2}+19 y^{2} \\
0\left(\bmod p^{2}\right) & \text { if }\left(\frac{p}{19}\right)=-1 .\end{cases} \\
& \text { If } p \neq 13 \text {, then } \\
& \sum_{k=0}^{p-1} \frac{\left(\begin{array}{c}
2 k \\
k
\end{array}\right)\left(\begin{array}{c}
3 k \\
k
\end{array}\right) T_{3 k}(106,1)}{312^{3 k}} \equiv \begin{cases}\left(\frac{78}{p}\right)\left(x^{2}-2 p\right)\left(\bmod p^{2}\right) & \text { if } 4 p=x^{2}+43 y^{2}, \\
0\left(\bmod p^{2}\right) & \text { if }\left(\frac{p}{43}\right)=-1 .\end{cases} \\
& \text { If } p \neq 73 \text {, then } \\
& \sum_{k=0}^{p-1} \frac{\left(\begin{array}{c}
2 k \\
k
\end{array}\right)\left(\begin{array}{c}
3 k \\
k
\end{array}\right) T_{3 k}(586,1)}{1752^{3 k}} \\
& \equiv \begin{cases}\left(\frac{438}{p}\right)\left(x^{2}-2 p\right)\left(\bmod p^{2}\right) & \text { if }\left(\frac{p}{67}\right)=1 \& 4 p=x^{2}+67 y^{2} \\
0\left(\bmod p^{2}\right) & \text { if }\left(\frac{p}{67}\right)=-1 .\end{cases} \\
& \text { If } p \neq 8893 \text {, then } \\
& \begin{aligned}
& \sum_{k=0}^{p-1} \frac{\left(\begin{array}{c}
2 k \\
k
\end{array}\right)\left(\begin{array}{c}
3 k \\
k
\end{array}\right) T_{3 k}(71146,1)}{213432^{3 k}} \\
\equiv & \begin{cases}\left(\frac{53358}{p}\right)\left(x^{2}-2 p\right)\left(\bmod p^{2}\right) & \text { if }\left(\frac{p}{163}\right)=1 \& 4 p=x^{2}+163 y^{2}, \\
0\left(\bmod p^{2}\right) & \text { if }\left(\frac{p}{163}\right)=-1 .\end{cases}
\end{aligned}
\end{aligned}
$$

Also,

$$
\begin{aligned}
& \sum_{k=0}^{p-1} \frac{\left(\begin{array}{c}
2 k \\
k
\end{array}\right)\left(\begin{array}{c}
3 k \\
k
\end{array}\right) T_{3 k}(2,-1)}{(-3456)^{k}} \equiv\left(\frac{-1}{p}\right) \sum_{k=0}^{p-1} \frac{\left(\begin{array}{c}
2 k \\
k
\end{array}\right)\left(\begin{array}{c}
3 k \\
k
\end{array}\right) T_{3 k}(2,9)}{24^{3 k}} \\
& \equiv \begin{cases}\left(\frac{2}{p}\right)\left(x^{2}-2 p\right)\left(\bmod p^{2}\right) & \text { if } p \equiv 1(\bmod 3) \& 4 p=x^{2}+27 y^{2}, \\
0\left(\bmod p^{2}\right) & \text { if } p \equiv 2(\bmod 3) .\end{cases}
\end{aligned}
$$


And

$$
\begin{aligned}
& \sum_{k=0}^{p-1}(15 k+2) \frac{\left(\begin{array}{c}
2 k \\
k
\end{array}\right)\left(\begin{array}{c}
3 k \\
k
\end{array}\right) T_{3 k}(2,-1)}{(-3456)^{k}} \\
\equiv & \begin{cases}2 p\left(\frac{2}{p}\right)\left(\bmod p^{2}\right) & \text { if } 3 \mid p-1 \text { and } 2 \text { is a cubis residue } \bmod p, \\
0(\bmod p) & \text { otherwise. }\end{cases}
\end{aligned}
$$

Remark 4.6. The imaginary quadratic field $\mathbb{Q}(\sqrt{-d})$ has class number one for $d=7,11,19,43,67,163$. We observe that if $p>3$ is a prime and $m$ is an integer with $m(3 m+8) \not \equiv 0(\bmod p)$ then

$$
\begin{aligned}
& \sum_{k=0}^{p-1} \frac{\left(\begin{array}{c}
2 k \\
k
\end{array}\right)\left(\begin{array}{c}
3 k \\
k
\end{array}\right) T_{3 k}(m+2,1)}{(3 m)^{3 k}} \\
\equiv & \left(\frac{-m(3 m+8)}{p}\right) \sum_{k=0}^{p-1} \frac{\left(\begin{array}{c}
2 k \\
k
\end{array}\right)\left(\begin{array}{c}
3 k \\
k
\end{array}\right)\left(\begin{array}{c}
6 k \\
3 k
\end{array}\right)}{(-9 m-24)^{3 k}}(\bmod p) .
\end{aligned}
$$

Conjecture 4.18. Let $p$ be an odd prime.

(i) When $p>5$ we have

$$
\begin{aligned}
& \sum_{k=0}^{p-1}\left(\frac{T_{k}\left(38,21^{2}\right)}{(-16)^{k}}\right)^{3} \equiv\left(\frac{-5}{p}\right) \sum_{k=0}^{p-1}\left(\frac{T_{k}\left(38,21^{2}\right)}{20^{k}}\right)^{3} \\
\equiv & \begin{cases}4 x^{2}-2 p\left(\bmod p^{2}\right) & \text { if }\left(\frac{2}{p}\right)=\left(\frac{p}{3}\right)=\left(\frac{p}{5}\right)=1, p=x^{2}+30 y^{2}, \\
12 x^{2}-2 p\left(\bmod p^{2}\right) & \text { if }\left(\frac{p}{3}\right)=1,\left(\frac{2}{p}\right)=\left(\frac{p}{5}\right)=-1, p=3 x^{2}+10 y^{2}, \\
2 p-8 x^{2}\left(\bmod p^{2}\right) & \text { if }\left(\frac{2}{p}\right)=1,\left(\frac{p}{3}\right)=\left(\frac{p}{5}\right)=-1, p=2 x^{2}+15 y^{2}, \\
20 x^{2}-2 p\left(\bmod p^{2}\right) & \text { if }\left(\frac{p}{5}\right)=1,\left(\frac{2}{p}\right)=\left(\frac{p}{3}\right)=-1, p=5 x^{2}+6 y^{2}, \\
p \delta_{p, 7}\left(\bmod p^{2}\right) & \text { if }\left(\frac{-30}{p}\right)=-1 .\end{cases}
\end{aligned}
$$

Also,

$$
\sum_{k=0}^{p-1}(28 k+15) \frac{T_{k}^{3}\left(38,21^{2}\right)}{(-16)^{3 k}} \equiv \frac{p}{7}\left(124-19\left(\frac{p}{3}\right)\right)\left(\bmod p^{2}\right) .
$$

If $p \neq 7$, then

$$
\sum_{k=0}^{p-1} \frac{\left(\begin{array}{c}
2 k \\
k
\end{array}\right) T_{k}^{2}(4,9)}{28^{2 k}} \equiv \sum_{k=0}^{p-1}\left(\frac{T_{k}\left(38,21^{2}\right)}{(-16)^{k}}\right)^{3}\left(\bmod p^{2}\right)
$$

and

$$
\sum_{k=0}^{p-1} \frac{24 k+5}{28^{2 k}}\left(\begin{array}{c}
2 k \\
k
\end{array}\right) T_{k}^{2}(4,9) \equiv p\left(\frac{-6}{p}\right)\left(4+\left(\frac{2}{p}\right)\right)\left(\bmod p^{2}\right) .
$$


(ii) When $p \neq 7$ we have

$$
\begin{aligned}
& \sum_{k=0}^{p-1}\left(\frac{T_{k}\left(110,57^{2}\right)}{32^{k}}\right)^{3} \equiv\left(\frac{-14}{p}\right) \sum_{k=0}^{p-1}\left(\frac{T_{k}\left(110,57^{2}\right)}{(-28)^{k}}\right)^{3} \\
& \equiv \begin{cases}4 x^{2}-2 p\left(\bmod p^{2}\right) & \text { if }\left(\frac{-2}{p}\right)=\left(\frac{p}{3}\right)=\left(\frac{p}{7}\right)=1, p=x^{2}+42 y^{2}, \\
8 x^{2}-2 p\left(\bmod p^{2}\right) & \text { if }\left(\frac{p}{7}\right)=1,\left(\frac{-2}{p}\right)=\left(\frac{p}{3}\right)=-1, p=2 x^{2}+21 y^{2}, \\
12 x^{2}-2 p\left(\bmod p^{2}\right) & \text { if }\left(\frac{-2}{p}\right)=1,\left(\frac{p}{3}\right)=\left(\frac{p}{7}\right)=-1, p=3 x^{2}+14 y^{2}, \\
24 x^{2}-2 p\left(\bmod p^{2}\right) & \text { if }\left(\frac{p}{3}\right)=1,\left(\frac{-2}{p}\right)=\left(\frac{p}{7}\right)=-1, p=6 x^{2}+7 y^{2}, \\
p \delta_{p, 19}\left(\bmod p^{2}\right) & \text { if }\left(\frac{-42}{p}\right)=-1 .\end{cases}
\end{aligned}
$$

Also,

$$
\sum_{k=0}^{p-1}(684 k+329) \frac{T_{k}^{3}\left(110,57^{2}\right)}{2^{15 k}} \equiv \frac{p}{19}\left(5160\left(\frac{-2}{p}\right)+1091\right)\left(\bmod p^{2}\right)
$$

If $p>3$ and $p \neq 11,19$, then

$$
\sum_{k=0}^{p-1} \frac{\left(\begin{array}{c}
2 k \\
k
\end{array}\right) T_{k}^{2}(5,1)}{22^{2 k}} \equiv \sum_{k=0}^{p-1}\left(\frac{T_{k}\left(110,57^{2}\right)}{32^{k}}\right)^{3}\left(\bmod p^{2}\right)
$$

and

$$
\sum_{k=0}^{p-1} \frac{560 k+71}{22^{2 k}}\left(\begin{array}{c}
2 k \\
k
\end{array}\right) T_{k}^{2}(5,1) \equiv \frac{p}{3}\left(280\left(\frac{p}{7}\right)-67\right)\left(\bmod p^{2}\right) .
$$

Conjecture 4.19. Let $p>3$ be a prime. When $p \neq 5$, we have

$$
\begin{aligned}
& \sum_{k=0}^{p-1} \frac{\left(\begin{array}{c}
2 k \\
k
\end{array}\right) T_{k}^{2}(9,12)}{900^{k}} \\
& \equiv \begin{cases}4 x^{2}-2 p\left(\bmod p^{2}\right) & \text { if }\left(\frac{-1}{p}\right)=\left(\frac{p}{3}\right)=\left(\frac{p}{11}\right)=1, p=x^{2}+33 y^{2}, \\
2 x^{2}-2 p\left(\bmod p^{2}\right) & \text { if }\left(\frac{-1}{p}\right)=1,\left(\frac{p}{3}\right)=\left(\frac{p}{11}\right)=-1,2 p=x^{2}+33 y^{2}, \\
12 x^{2}-2 p\left(\bmod p^{2}\right) & \text { if }\left(\frac{p}{11}\right)=1,\left(\frac{-1}{p}\right)=\left(\frac{p}{3}\right)=-1, p=3 x^{2}+11 y^{2}, \\
6 x^{2}-2 p\left(\bmod p^{2}\right) & \text { if }\left(\frac{p}{3}\right)=1,\left(\frac{-1}{p}\right)=\left(\frac{p}{11}\right)=-1,2 p=3 x^{2}+11 y^{2}, \\
0\left(\bmod p^{2}\right) & \text { if }\left(\frac{-33}{p}\right)=-1 .\end{cases}
\end{aligned}
$$


Provided $p \neq 29$, we have

$$
\begin{aligned}
& \sum_{k=0}^{p-1} \frac{\left(\begin{array}{c}
2 k \\
k
\end{array}\right) T_{k}^{2}(171,-171)}{(-5177196)^{k}} \\
& \equiv \begin{cases}4 x^{2}-2 p\left(\bmod p^{2}\right) & \text { if }\left(\frac{-1}{p}\right)=\left(\frac{p}{7}\right)=\left(\frac{p}{19}\right)=1, p=x^{2}+133 y^{2}, \\
2 x^{2}-2 p\left(\bmod p^{2}\right) & \text { if }\left(\frac{p}{7}\right)=1,\left(\frac{-1}{p}\right)=\left(\frac{p}{19}\right)=-1,2 p=x^{2}+133 y^{2}, \\
2 p-28 x^{2}\left(\bmod p^{2}\right) & \text { if }\left(\frac{p}{19}\right)=1,\left(\frac{-1}{p}\right)=\left(\frac{p}{7}\right)=-1, p=7 x^{2}+19 y^{2}, \\
2 p-14 x^{2}\left(\bmod p^{2}\right) & \text { if }\left(\frac{-1}{p}\right)=1,\left(\frac{p}{7}\right)=\left(\frac{p}{19}\right)=-1,2 p=7 x^{2}+19 y^{2}, \\
0\left(\bmod p^{2}\right) & \text { if }\left(\frac{-133}{p}\right)=-1 .\end{cases}
\end{aligned}
$$

Conjecture 4.20. Let $p$ be an odd prime. When $p \neq 23$, we have

$$
\begin{aligned}
& \sum_{k=0}^{p-1} \frac{\left(\begin{array}{c}
2 k \\
k
\end{array}\right) T_{k}^{2}(7,1)}{46^{2 k}} \\
& \equiv \begin{cases}4 x^{2}-2 p\left(\bmod p^{2}\right) & \text { if }\left(\frac{2}{p}\right)=\left(\frac{p}{5}\right)=\left(\frac{p}{7}\right)=1, p=x^{2}+70 y^{2}, \\
8 x^{2}-2 p\left(\bmod p^{2}\right) & \text { if }\left(\frac{p}{7}\right)=1,\left(\frac{2}{p}\right)=\left(\frac{p}{5}\right)=-1, p=2 x^{2}+35 y^{2}, \\
20 x^{2}-2 p\left(\bmod p^{2}\right) & \text { if }\left(\frac{p}{5}\right)=1,\left(\frac{2}{p}\right)=\left(\frac{p}{7}\right)=-1, p=5 x^{2}+14 y^{2}, \\
2 p-28 x^{2}\left(\bmod p^{2}\right) & \text { if }\left(\frac{2}{p}\right)=1,\left(\frac{p}{5}\right)=\left(\frac{p}{7}\right)=-1, p=7 x^{2}+10 y^{2}, \\
0\left(\bmod p^{2}\right) & \text { if }\left(\frac{-70}{p}\right)=-1 .\end{cases}
\end{aligned}
$$

Provided $p \neq 3,7,11,17,31$, we have

$$
\begin{aligned}
& \sum_{k=0}^{p-1} \frac{\left(\begin{array}{c}
2 k \\
k
\end{array}\right) T_{k}^{2}(73,576)}{434^{2 k}} \\
& \equiv \begin{cases}4 x^{2}-2 p\left(\bmod p^{2}\right) & \text { if }\left(\frac{2}{p}\right)=\left(\frac{p}{3}\right)=\left(\frac{p}{17}\right)=1, p=x^{2}+102 y^{2}, \\
8 x^{2}-2 p\left(\bmod p^{2}\right) & \text { if }\left(\frac{p}{17}\right)=1,\left(\frac{2}{p}\right)=\left(\frac{p}{3}\right)=-1, p=2 x^{2}+51 y^{2}, \\
12 x^{2}-2 p\left(\bmod p^{2}\right) & \text { if }\left(\frac{p}{3}\right)=1,\left(\frac{2}{p}\right)=\left(\frac{p}{17}\right)=-1, p=3 x^{2}+34 y^{2}, \\
24 x^{2}-2 p\left(\bmod p^{2}\right) & \text { if }\left(\frac{2}{p}\right)=1,\left(\frac{p}{3}\right)=\left(\frac{p}{17}\right)=-1, p=6 x^{2}+17 y^{2}, \\
0\left(\bmod p^{2}\right) & \text { if }\left(\frac{-102}{p}\right)=-1 .\end{cases}
\end{aligned}
$$


Conjecture 4.21. Let $p>3$ be a prime. If $p \neq 23$, then

$$
\begin{aligned}
& \sum_{k=0}^{p-1} \frac{\left(\begin{array}{c}
2 k \\
k
\end{array}\right) T_{k}^{2}\left(23,7^{4}\right)}{46^{2 k}} \\
& \equiv \begin{cases}4 x^{2}-2 p\left(\bmod p^{2}\right) & \text { if }\left(\frac{2}{p}\right)=\left(\frac{p}{3}\right)=\left(\frac{p}{13}\right)=1, p=x^{2}+78 y^{2}, \\
8 x^{2}-2 p\left(\bmod p^{2}\right) & \text { if }\left(\frac{2}{p}\right)=1,\left(\frac{p}{3}\right)=\left(\frac{p}{13}\right)=-1, p=2 x^{2}+39 y^{2}, \\
2 p-12 x^{2}\left(\bmod p^{2}\right) & \text { if }\left(\frac{p}{13}\right)=1,\left(\frac{2}{p}\right)=\left(\frac{p}{3}\right)=-1, p=3 x^{2}+26 y^{2}, \\
2 p-24 x^{2}\left(\bmod p^{2}\right) & \text { if }\left(\frac{p}{3}\right)=1,\left(\frac{2}{p}\right)=\left(\frac{p}{13}\right)=-1, p=6 x^{2}+13 y^{2}, \\
p \delta_{p, 7}\left(\bmod p^{2}\right) & \text { if }\left(\frac{-78}{p}\right)=-1,\end{cases}
\end{aligned}
$$

where $\delta_{m, n}$ takes 1 or 0 according as $m=n$ or not. If $p \neq 5$, then

$$
\begin{aligned}
& \sum_{k=0}^{p-1} \frac{T_{k}^{3}\left(1298,651^{2}\right)}{(-100)^{3 k}} \\
\equiv & \begin{cases}4 x^{2}-2 p\left(\bmod p^{2}\right) & \text { if }\left(\frac{2}{p}\right)=\left(\frac{p}{3}\right)=\left(\frac{p}{13}\right)=1, p=x^{2}+78 y^{2}, \\
2 p-8 x^{2}\left(\bmod p^{2}\right) & \text { if }\left(\frac{2}{p}\right)=1,\left(\frac{p}{3}\right)=\left(\frac{p}{13}\right)=-1, p=2 x^{2}+39 y^{2}, \\
12 x^{2}-2 p\left(\bmod p^{2}\right) & \text { if }\left(\frac{p}{13}\right)=1,\left(\frac{2}{p}\right)=\left(\frac{p}{3}\right)=-1, p=3 x^{2}+26 y^{2}, \\
2 p-24 x^{2}\left(\bmod p^{2}\right) & \text { if }\left(\frac{p}{3}\right)=1,\left(\frac{2}{p}\right)=\left(\frac{p}{13}\right)=-1, p=6 x^{2}+13 y^{2}, \\
p\left(\delta_{p, 7}+\delta_{p, 31}\right)\left(\bmod p^{2}\right) & \text { if }\left(\frac{-78}{p}\right)=-1 .\end{cases}
\end{aligned}
$$

Conjecture 4.22. Let $p \neq 2,7,11$ be a prime. Then

$$
\begin{aligned}
& \sum_{k=0}^{p-1} \frac{\left(\begin{array}{c}
2 k \\
k
\end{array}\right) T_{k}^{2}(7,81)}{14^{2 k}} \equiv\left(\frac{p}{11}\right) \sum_{k=0}^{p-1} \frac{\left(\begin{array}{c}
2 k \\
k
\end{array}\right) T_{k}^{2}(20,1)}{28^{2 k}} \\
\equiv & \begin{cases}4 x^{2}-2 p\left(\bmod p^{2}\right) & \text { if }\left(\frac{-11}{p}\right)=\left(\frac{2}{p}\right)=1 \& p=x^{2}+22 y^{2}, \\
8 x^{2}-2 p\left(\bmod p^{2}\right) & \text { if }\left(\frac{-11}{p}\right)=\left(\frac{2}{p}\right)=-1 \& p=2 x^{2}+11 y^{2}, \\
0\left(\bmod p^{2}\right) & \text { if }\left(\frac{-11}{p}\right)=-\left(\frac{2}{p}\right) .\end{cases}
\end{aligned}
$$

Conjecture 4.23. Let $p \neq 2,7$ be a prime. Then

$$
\sum_{k=0}^{p-1} \frac{\left(\begin{array}{c}
2 k \\
k
\end{array}\right) T_{k}^{2}(6,2)}{450^{k}} \equiv \begin{cases}4 x^{2}-2 p\left(\bmod p^{2}\right) & \text { if }\left(\frac{p}{7}\right)=1 \& p=x^{2}+7 y^{2} \\
0\left(\bmod p^{2}\right) & \text { if }\left(\frac{p}{7}\right)=-1\end{cases}
$$

and

$$
\sum_{k=0}^{p-1} \frac{221 k+28}{450^{k}}\left(\begin{array}{c}
2 k \\
k
\end{array}\right) T_{k}^{2}(6,2) \equiv \frac{4 p}{7}\left(72\left(\frac{-1}{p}\right)-23\right)\left(\bmod p^{2}\right) .
$$


Conjecture 4.24. Let $p>3$ be a prime. Then

$$
\begin{aligned}
& \sum_{k=0}^{p-1} \frac{\left(\begin{array}{c}
2 k \\
k
\end{array}\right) T_{k}^{2}(7,12)}{4^{k}} \equiv \sum_{k=0}^{p-1} \frac{\left(\begin{array}{c}
2 k \\
k
\end{array}\right) T_{2 k}^{2}(3,3)}{36^{k}} \\
\equiv & \begin{cases}4 x^{2}-2 p\left(\bmod p^{2}\right) & \text { if } p \equiv 1(\bmod 12) \& p=x^{2}+9 y^{2}, \\
4 x y\left(\bmod p^{2}\right) & \text { if } p \equiv 5(\bmod 12) \& p=x^{2}+y^{2}(3 \mid x-y), \\
0\left(\bmod p^{2}\right) & \text { if } p \equiv 3(\bmod 4) .\end{cases}
\end{aligned}
$$

Conjecture 4.25. Let $p$ be an odd prime. Then

$$
\begin{aligned}
& \sum_{k=0}^{p-1} \frac{\left(\begin{array}{c}
2 k \\
k
\end{array}\right) T_{k}^{2}(19,-20)}{22^{2 k}} \equiv \sum_{k=0}^{p-1} \frac{\left(\begin{array}{c}
2 k \\
k
\end{array}\right) T_{2 k}^{2}(9,20)}{4^{k}} \\
\equiv & \begin{cases}4 x^{2}-2 p\left(\bmod p^{2}\right) & \text { if }\left(\frac{-1}{p}\right)=\left(\frac{p}{5}\right)=1 \& p=x^{2}+y^{2}(5 \nmid x), \\
4 x y\left(\bmod p^{2}\right) & \text { if }\left(\frac{-1}{p}\right)=-\left(\frac{p}{5}\right)=1 \& p=x^{2}+y^{2}(5 \mid x-y), \\
0\left(\bmod p^{2}\right) & \text { if } p \equiv 3(\bmod 4) \& p \neq 11 .\end{cases}
\end{aligned}
$$

We have many conjectures similar to Conjectures 4.18-4.25. For example, we find that $\sum_{k=0}^{p-1}\left(\begin{array}{c}2 k \\ k\end{array}\right) T_{k}^{2}(b, c) / m^{k} \bmod p^{2}$ is related to the representation $p=x^{2}+d y^{2}$ if $(b, c, m ; d)$ is among

$$
\begin{gathered}
(5,4,4 ; 10),(3,-4,36 ; 13),\left(5,4,14^{2} ; 30\right), \\
\left(7,1,14^{2} ; 30\right),\left(7,28,14^{2} ; 21\right),\left(11,49,22^{2} ; 42\right) .
\end{gathered}
$$

Though we will not list many other conjectures similar to Conjectures 4.4-4.25, the above conjectures should convince the reader that our conjectural series for $1 / \pi$ in the next section are indeed reasonable in view of the corresponding congruences.

\section{DuAlities AND NEW SERIES FOR $1 / \pi$}

As mentioned in Section 1, for $b>0$ and $c>0$ the main term of $T_{n}(b, c)$ as $n \rightarrow+\infty$ is

$$
f_{n}(b, c):=\frac{(b+2 \sqrt{c})^{n+1 / 2}}{2 \sqrt[4]{c} \sqrt{n \pi}} .
$$

Here we formulate a further refinement of this.

Conjecture 5.1. For any positive real numbers $b$ and $c$, we have

$$
T_{n}(b, c)=f_{n}(b, c)\left(1+\frac{b-4 \sqrt{c}}{16 n \sqrt{c}}+O\left(\frac{1}{n^{2}}\right)\right)
$$


as $n \rightarrow+\infty$. If $c>0$ and $b=4 \sqrt{c}$, then

$$
\frac{T_{n}(b, c)}{\sqrt{c}^{n}}=T_{n}(4,1)=\frac{3 \times 6^{n}}{\sqrt{6 n \pi}}\left(1+\frac{1}{8 n^{2}}+\frac{15}{64 n^{3}}+\frac{21}{32 n^{4}}+O\left(\frac{1}{n^{5}}\right)\right) .
$$

If $c<0$ and $b \in \mathbb{R}$ then

$$
\lim _{n \rightarrow \infty} \sqrt[n]{\left|T_{n}(b, c)\right|}=\sqrt{b^{2}-4 c}
$$

Let $p$ be an odd prime. Z.-H. Sun [S1] proved the congruence

$$
\sum_{k=0}^{p-1} \frac{\left(\begin{array}{c}
2 k \\
k
\end{array}\right)^{2}}{16^{k}} x^{k} \equiv\left(\frac{-1}{p}\right) \sum_{k=0}^{p-1} \frac{\left(\begin{array}{c}
2 k \\
k
\end{array}\right)^{2}}{16^{k}}(1-x)^{k}\left(\bmod p^{2}\right)
$$

via Legendre polynomials; in fact this follows from the well-known identity $P_{n}(-x)=(-1)^{n} P_{n}(x)$ with $n=(p-1) / 2$. In [Su8] the author managed to show the following congruences via the Zeilberger algorithm:

$$
\begin{aligned}
& \sum_{k=0}^{p-1} \frac{\left(\begin{array}{c}
2 k \\
k
\end{array}\right)\left(\begin{array}{c}
4 k \\
2 k
\end{array}\right)}{64^{k}} x^{k} \equiv\left(\frac{-2}{p}\right) \sum_{k=0}^{p-1} \frac{\left(\begin{array}{c}
2 k \\
k
\end{array}\right)\left(\begin{array}{c}
4 k \\
2 k
\end{array}\right)}{64^{k}}(1-x)^{k}\left(\bmod p^{2}\right), \\
& \sum_{k=0}^{p-1} \frac{\left(\begin{array}{c}
2 k \\
k
\end{array}\right)\left(\begin{array}{c}
3 k \\
k
\end{array}\right)}{27^{k}} x^{k} \equiv\left(\frac{p}{3}\right) \sum_{k=0}^{p-1} \frac{\left(\begin{array}{c}
2 k \\
k
\end{array}\right)\left(\begin{array}{c}
3 k \\
k
\end{array}\right)}{27^{k}}(1-x)^{k}\left(\bmod p^{2}\right) \quad(p \neq 3), \\
& \sum_{k=0}^{p-1} \frac{\left(\begin{array}{c}
3 k \\
k
\end{array}\right)\left(\begin{array}{c}
6 k \\
3 k
\end{array}\right)}{432^{k}} x^{k} \equiv\left(\frac{-1}{p}\right) \sum_{k=0}^{p-1} \frac{\left(\begin{array}{c}
3 k \\
k
\end{array}\right)\left(\begin{array}{c}
6 k \\
3 k
\end{array}\right)}{432^{k}}(1-x)^{k}\left(\bmod p^{2}\right) \quad(p \neq 3) .
\end{aligned}
$$

Our following result on dualities was motivated by (38)-(41).

Theorem 5.1. Let $p$ be an odd prime and let $b, c$ and $m \neq \equiv 0(\bmod p)$ be rational $p$-adic integers. Then

$$
\begin{aligned}
& \sum_{k=0}^{p-1} \frac{\left(\begin{array}{c}
2 k \\
k
\end{array}\right)^{2}}{(16 m)^{k}} T_{k}(b, c) \equiv\left(\frac{-1}{p}\right) \sum_{k=0}^{p-1} \frac{\left(\begin{array}{c}
2 k \\
k
\end{array}\right)^{2}}{(16 m)^{k}} T_{k}(m-b, c)\left(\bmod p^{2}\right), \\
& \sum_{k=0}^{p-1} \frac{\left(\begin{array}{c}
2 k \\
k
\end{array}\right)\left(\begin{array}{c}
4 k \\
2 k
\end{array}\right)}{(64 m)^{k}} T_{k}(b, c) \equiv\left(\frac{-2}{p}\right) \sum_{k=0}^{p-1} \frac{\left(\begin{array}{c}
2 k \\
k
\end{array}\right)\left(\begin{array}{c}
4 k \\
2 k
\end{array}\right)}{(64 m)^{k}} T_{k}(m-b, c)\left(\bmod p^{2}\right),
\end{aligned}
$$


Provided $p>3$ we also have

$$
\begin{aligned}
& \sum_{k=0}^{p-1} \frac{\left(\begin{array}{c}
2 k \\
k
\end{array}\right)\left(\begin{array}{c}
3 k \\
k
\end{array}\right)}{(27 m)^{k}} T_{k}(b, c) \equiv\left(\frac{p}{3}\right) \sum_{k=0}^{p-1} \frac{\left(\begin{array}{c}
2 k \\
k
\end{array}\right)\left(\begin{array}{c}
3 k \\
k
\end{array}\right)}{(27 m)^{k}} T_{k}(m-b, c)\left(\bmod p^{2}\right), \\
& \sum_{k=0}^{p-1} \frac{\left(\begin{array}{c}
3 k \\
k
\end{array}\right)\left(\begin{array}{c}
6 k \\
3 k
\end{array}\right)}{(432 m)^{k}} T_{k}(b, c) \equiv\left(\frac{-1}{p}\right) \sum_{k=0}^{p-1} \frac{\left(\begin{array}{c}
3 k \\
k
\end{array}\right)\left(\begin{array}{c}
6 k \\
3 k
\end{array}\right)}{(432 m)^{k}} T_{k}(m-b, c)\left(\bmod p^{2}\right) .
\end{aligned}
$$

Proof. Since the proofs of (42)-(45) are very similar, we just show (43) in detail.

For $d=0, \ldots, p-1$, by taking differentiations of both sides (39) $d$ times we get

$$
\sum_{k=0}^{p-1} \frac{\left(\begin{array}{c}
2 k \\
k
\end{array}\right)\left(\begin{array}{c}
4 k \\
2 k
\end{array}\right)}{64^{k}}\left(\begin{array}{c}
k \\
d
\end{array}\right) x^{k-d} \equiv\left(\frac{-2}{p}\right) \sum_{k=0}^{p-1} \frac{\left(\begin{array}{c}
2 k \\
k
\end{array}\right)\left(\begin{array}{c}
4 k \\
2 k
\end{array}\right)}{64^{k}}(-1)^{d}\left(\begin{array}{l}
k \\
d
\end{array}\right)(1-x)^{k-d}\left(\bmod p^{2}\right) .
$$

In view of this, we have

$$
\begin{aligned}
& \sum_{k=0}^{p-1} \frac{\left(\begin{array}{c}
2 k \\
k
\end{array}\right)\left(\begin{array}{l}
4 k \\
2 k
\end{array}\right)}{(64 m)^{k}} T_{k}(b, c) \\
= & \sum_{k=0}^{p-1} \frac{\left(\begin{array}{c}
2 k \\
k
\end{array}\right)\left(\begin{array}{l}
4 k \\
2 k
\end{array}\right)}{(64 m)^{k}} \sum_{j=0}^{\lfloor k / 2\rfloor}\left(\begin{array}{c}
k \\
2 j
\end{array}\right)\left(\begin{array}{c}
2 j \\
j
\end{array}\right) b^{k-2 j} c^{j} \\
= & \sum_{j=0}^{p-1}\left(\begin{array}{c}
2 j \\
j
\end{array}\right) \frac{c^{j}}{m^{2 j}} \sum_{k=0}^{p-1} \frac{\left(\begin{array}{c}
2 k \\
k
\end{array}\right)\left(\begin{array}{l}
4 k \\
2 k
\end{array}\right)}{64^{k}}\left(\begin{array}{c}
k \\
2 j
\end{array}\right)\left(\frac{b}{m}\right)^{k-2 j} \\
\equiv & \sum_{j=0}^{p-1}\left(\begin{array}{c}
2 j \\
j
\end{array}\right) \frac{c^{j}}{m^{2 j}}\left(\frac{-2}{p}\right) \sum_{k=0}^{p-1} \frac{\left(\begin{array}{c}
2 k \\
k
\end{array}\right)\left(\begin{array}{c}
4 k \\
2 k
\end{array}\right)}{64^{k}}\left(\begin{array}{c}
k \\
2 j
\end{array}\right)\left(1-\frac{b}{m}\right)^{k-2 j} \\
= & \left(\frac{-2}{p}\right) \sum_{k=0}^{p-1} \frac{\left(\begin{array}{c}
2 k \\
k
\end{array}\right)\left(\begin{array}{c}
4 k \\
2 k
\end{array}\right)}{(64 m)^{k}} \sum_{j=0}^{\lfloor k / 2\rfloor}\left(\begin{array}{c}
k \\
2 j
\end{array}\right)\left(\begin{array}{c}
2 j \\
j
\end{array}\right)(m-b)^{k-2 j} c^{j} \\
= & \left(\frac{-2}{p}\right) \sum_{k=0}^{p-1} \frac{\left(\begin{array}{c}
2 k \\
k
\end{array}\right)\left(\begin{array}{c}
4 k \\
2 k
\end{array}\right)}{(64 m)^{k}} T_{k}(m-b, c)\left(\bmod p^{2}\right) .
\end{aligned}
$$

The proof of Theorem 5.1 is now complete.

Example 1. Let $p$ be an odd prime. By (42) we have

$$
\left(\frac{-1}{p}\right) \sum_{k=0}^{p-1} \frac{\left(\begin{array}{c}
2 k \\
k
\end{array}\right)^{2} T_{k}(5,4)}{16^{k}} \equiv \sum_{k=0}^{p-1} \frac{\left(\begin{array}{c}
2 k \\
k
\end{array}\right)^{2} T_{k}(-4,4)}{16^{k}}=\sum_{k=0}^{p-1} \frac{\left(\begin{array}{c}
2 k \\
k
\end{array}\right)^{3}}{(-8)^{k}}\left(\bmod p^{2}\right) .
$$


The author [Su4] conjectured that

$$
\sum_{k=0}^{p-1} \frac{\left(\begin{array}{c}
2 k \\
k
\end{array}\right)^{3}}{(-8)^{k}} \equiv \begin{cases}4 x^{2}-2 p\left(\bmod p^{2}\right) & \text { if } p=x^{2}+y^{2}(2 \nmid x), \\
0\left(\bmod p^{2}\right) & \text { if } p \equiv 3(\bmod 4),\end{cases}
$$

and this was recently confirmed by Z.-H. Sun [S2]. When $p>3$, by (44) we have

$$
\begin{aligned}
& \left(\frac{p}{3}\right) \sum_{k=0}^{p-1} \frac{\left(\begin{array}{c}
2 k \\
k
\end{array}\right)\left(\begin{array}{c}
3 k \\
k
\end{array}\right) T_{k}(3,1)}{27^{k}} \\
\equiv & \sum_{k=0}^{p-1} \frac{\left(\begin{array}{c}
2 k \\
k
\end{array}\right)\left(\begin{array}{c}
3 k \\
k
\end{array}\right) T_{k}(-2,1)}{27^{k}}=\sum_{k=0}^{p-1} \frac{\left(\begin{array}{c}
2 k \\
k
\end{array}\right)^{2}\left(\begin{array}{c}
3 k \\
k
\end{array}\right)}{(-27)^{k}}\left(\bmod p^{2}\right)
\end{aligned}
$$

the reader may consult [Su4, Conjecture 5.6] for $\sum_{k=0}^{p-1}\left(\begin{array}{c}2 k \\ k\end{array}\right)^{2}\left(\begin{array}{c}3 k \\ k\end{array}\right) /(-27)^{k}$ $\bmod p^{2}$.

Based on our investigations of congruences on sums of central binomial coefficients and central trinomial coefficients, and the author's philosophy about series for $1 / \pi$ stated in [Su7], we raise 61 conjectural series for $1 / \pi$ of the following seven new types with $a, b, c, d, m$ integers and $a b c d\left(b^{2}-4 c\right) m$ nonzero.

$$
\begin{aligned}
& \text { Type I. } \sum_{k=0}^{\infty}(a+d k)\left(\begin{array}{c}
2 k \\
k
\end{array}\right){ }^{2} T_{k}(b, c) / m^{k} . \\
& \text { Type II. } \sum_{k=0}^{\infty}(a+d k)\left(\begin{array}{c}
2 k \\
k
\end{array}\right)\left(\begin{array}{c}
3 k \\
k
\end{array}\right) T_{k}(b, c) / m^{k} . \\
& \text { Type III. } \sum_{k=0}^{\infty}(a+d k)\left(\begin{array}{c}
4 k \\
2 k
\end{array}\right)\left(\begin{array}{c}
2 k \\
k
\end{array}\right) T_{k}(b, c) / m^{k} . \\
& \text { Type IV. } \sum_{k=0}^{\infty}(a+d k)\left(\begin{array}{c}
2 k \\
k
\end{array}\right) T_{2 k}(b, c) / m^{k} . \\
& \text { Type V. } \sum_{k=0}^{\infty}(a+d k)\left(\begin{array}{c}
2 k \\
k
\end{array}\right)\left(\begin{array}{c}
3 k \\
k
\end{array}\right) T_{3 k}(b, c) / m^{k} . \\
& \text { Type VI. } \sum_{k=0}^{\infty}(a+d k) T_{k}(b, c)^{3} / m^{k} . \\
& \text { Type VII. } \sum_{k=0}^{\infty}(a+d k)\left(\begin{array}{c}
2 k \\
k
\end{array}\right) T_{k}(b, c)^{2} / m^{k} .
\end{aligned}
$$

Recall that a series $\sum_{k=0}^{\infty} a_{k}$ is said to converge at a geometric rate with ratio $r$ if $\lim _{k \rightarrow+\infty} a_{k+1} / a_{k}=r \in(0,1)$. All the series in Conjectures I-VII below converge at geometrical rates, and they were found by the author in 2011 except that (IV19)-(IV21) were discovered in 2012.

Conjecture I. We have the following identities:

$$
\begin{gathered}
\sum_{k=0}^{\infty} \frac{30 k+7}{(-256)^{k}}\left(\begin{array}{c}
2 k \\
k
\end{array}\right)^{2} T_{k}(1,16)=\frac{24}{\pi}, \\
\sum_{k=0}^{\infty} \frac{30 k+7}{(-1024)^{k}}\left(\begin{array}{c}
2 k \\
k
\end{array}\right)^{2} T_{k}(34,1)=\frac{12}{\pi},
\end{gathered}
$$




$$
\begin{aligned}
& \sum_{k=0}^{\infty} \frac{30 k-1}{4096^{k}}\left(\begin{array}{c}
2 k \\
k
\end{array}\right)^{2} T_{k}(194,1)=\frac{80}{\pi} \\
& \sum_{k=0}^{\infty} \frac{42 k+5}{4096^{k}}\left(\begin{array}{c}
2 k \\
k
\end{array}\right)^{2} T_{k}(62,1)=\frac{16 \sqrt{3}}{\pi} .
\end{aligned}
$$

Remark 5.1. (I1) was the first identity for $1 / \pi$ involving generalized central trinomial coefficients; it was discovered by the author on Jan. 2, 2011. Different from classical Ramanujan-type series for $1 / \pi$ (cf. N. D. Baruah and B. C. Berndt [BB], and Berndt [Be, pp. 353-354]) and their known generalizations (see, e.g., S. Cooper $[\mathrm{C}]$ ), the two coefficients in the linear part $30 k-1$ of (I3) have different signs, and also its corresponding $p$-adic congruence (with $p>3$ a prime) involves two Legendre symbols:

$$
\sum_{k=0}^{p-1}(30 k-1) \frac{\left(\begin{array}{c}
2 k \\
k
\end{array}\right)^{2} T_{k}(194,1)}{4096^{k}} \equiv p\left(5\left(\frac{-1}{p}\right)-6\left(\frac{3}{p}\right)\right)\left(\bmod p^{2}\right)
$$

Conjecture II. We have

$$
\begin{aligned}
\sum_{k=0}^{\infty} \frac{15 k+2}{972^{k}}\left(\begin{array}{c}
2 k \\
k
\end{array}\right)\left(\begin{array}{c}
3 k \\
k
\end{array}\right) T_{k}(18,6) & =\frac{45 \sqrt{3}}{4 \pi}, \\
\sum_{k=0}^{\infty} \frac{91 k+12}{10^{3 k}}\left(\begin{array}{c}
2 k \\
k
\end{array}\right)\left(\begin{array}{c}
3 k \\
k
\end{array}\right) T_{k}(10,1) & =\frac{75 \sqrt{3}}{2 \pi}, \\
\sum_{k=0}^{\infty} \frac{15 k-4}{18^{3 k}}\left(\begin{array}{c}
2 k \\
k
\end{array}\right)\left(\begin{array}{c}
3 k \\
k
\end{array}\right) T_{k}(198,1) & =\frac{135 \sqrt{3}}{2 \pi}, \\
\sum_{k=0}^{\infty} \frac{42 k-41}{30^{3 k}}\left(\begin{array}{c}
2 k \\
k
\end{array}\right)\left(\begin{array}{c}
3 k \\
k
\end{array}\right) T_{k}(970,1) & =\frac{525 \sqrt{3}}{\pi}, \\
\sum_{k=0}^{\infty} \frac{18 k+1}{30^{3 k}}\left(\begin{array}{c}
2 k \\
k
\end{array}\right)\left(\begin{array}{c}
3 k \\
k
\end{array}\right) T_{k}(730,729) & =\frac{25 \sqrt{3}}{\pi}, \\
\sum_{k=0}^{\infty} \frac{6930 k+559}{102^{3 k}}\left(\begin{array}{c}
2 k \\
k
\end{array}\right)\left(\begin{array}{c}
3 k \\
k
\end{array}\right) T_{k}(102,1) & =\frac{1445 \sqrt{6}}{2 \pi},
\end{aligned}
$$


and

$$
\begin{aligned}
\sum_{k=0}^{\infty} \frac{222105 k+15724}{198^{3 k}}\left(\begin{array}{c}
2 k \\
k
\end{array}\right)\left(\begin{array}{c}
3 k \\
k
\end{array}\right) T_{k}(198,1) & =\frac{114345 \sqrt{3}}{4 \pi}, \\
\sum_{k=0}^{\infty} \frac{390 k-3967}{102^{3 k}}\left(\begin{array}{c}
2 k \\
k
\end{array}\right)\left(\begin{array}{c}
3 k \\
k
\end{array}\right) T_{k}(39202,1) & =\frac{56355 \sqrt{3}}{\pi}, \\
\sum_{k=0}^{\infty} \frac{210 k-7157}{198^{3 k}}\left(\begin{array}{c}
2 k \\
k
\end{array}\right)\left(\begin{array}{c}
3 k \\
k
\end{array}\right) T_{k}(287298,1) & =\frac{114345 \sqrt{3}}{\pi}, \\
\sum_{k=0}^{\infty} \frac{45 k+7}{24^{3 k}}\left(\begin{array}{c}
2 k \\
k
\end{array}\right)\left(\begin{array}{c}
3 k \\
k
\end{array}\right) T_{k}(26,729) & =\frac{8}{3 \pi}(3 \sqrt{3}+\sqrt{15}), \\
\sum_{k=0}^{\infty} \frac{9 k+2}{(-5400)^{k}}\left(\begin{array}{c}
2 k \\
k
\end{array}\right)\left(\begin{array}{c}
3 k \\
k
\end{array}\right) T_{k}(70,3645) & =\frac{15 \sqrt{3}+\sqrt{15}}{6 \pi}, \\
\sum_{k=0}^{\infty} \frac{63 k+11}{(-13500)^{k}}\left(\begin{array}{c}
2 k \\
k
\end{array}\right)\left(\begin{array}{c}
3 k \\
k
\end{array}\right) T_{k}(40,1458) & =\frac{25}{12 \pi}(3 \sqrt{3}+4 \sqrt{6}) .
\end{aligned}
$$

Remark 5.2. . In view of (44), we may view (II9) as the dual of (II7) since $198^{3} / 27-198=287298$. The series in (II7) converges rapidly at a geometric rate with ratio $25 / 35937$, but the series in (II9) converges very slow at a geometric rate with ratio $71825 / 71874$. (II2), (II9) and (II10) were motivated by the following congruences (with $p>3$ a prime):

$$
\begin{aligned}
& \sum_{k=0}^{p-1} \frac{\left(\begin{array}{c}
2 k \\
k
\end{array}\right)\left(\begin{array}{c}
3 k \\
k
\end{array}\right) T_{k}(10,1)}{10^{3 k}} \\
& \equiv \begin{cases}4 x^{2}-2 p\left(\bmod p^{2}\right) & \text { if } p \equiv 1,3(\bmod 8) \& p=x^{2}+2 y^{2}, \\
0\left(\bmod p^{2}\right) & \text { if }\left(\frac{-2}{p}\right)=-1, \text { i.e., } p \equiv 5,7(\bmod 8),\end{cases} \\
& \sum_{k=0}^{p-1}(91 k+12) \frac{\left(\begin{array}{c}
2 k \\
k
\end{array}\right)\left(\begin{array}{c}
3 k \\
k
\end{array}\right) T_{k}(10,1)}{10^{3 k}} \\
& \equiv \frac{3 p}{2}\left(9\left(\frac{-3}{p}\right)-1\right)\left(\bmod p^{2}\right)(p \neq 5) ;
\end{aligned}
$$




$$
\begin{aligned}
& \sum_{k=0}^{p-1}(210 k-7157) \frac{\left(\begin{array}{c}
2 k \\
k
\end{array}\right)\left(\begin{array}{c}
3 k \\
k
\end{array}\right) T_{k}(287298,1)}{198^{3 k}} \\
& \equiv p\left(35\left(\frac{-3}{p}\right)-7192\right)\left(\bmod p^{2}\right) \quad(p \neq 11) ; \\
& \sum_{k=0}^{p-1} \frac{45 k+7}{24^{3 k}}\left(\begin{array}{c}
2 k \\
k
\end{array}\right)\left(\begin{array}{c}
3 k \\
k
\end{array}\right) T_{k}(26,729) \\
& \equiv \frac{p}{2}\left(9\left(\frac{-3}{p}\right)+5\left(\frac{-15}{p}\right)\right)\left(\bmod p^{2}\right) .
\end{aligned}
$$

Conjecture III. We have the following formulae:

$$
\begin{aligned}
& \sum_{k=0}^{\infty} \frac{85 k+2}{66^{2 k}}\left(\begin{array}{c}
4 k \\
2 k
\end{array}\right)\left(\begin{array}{c}
2 k \\
k
\end{array}\right) T_{k}(52,1)=\frac{33 \sqrt{33}}{\pi}, \\
& \sum_{k=0}^{\infty} \frac{28 k+5}{\left(-96^{2}\right)^{k}}\left(\begin{array}{c}
4 k \\
2 k
\end{array}\right)\left(\begin{array}{c}
2 k \\
k
\end{array}\right) T_{k}(110,1)=\frac{3 \sqrt{6}}{\pi}, \\
& \sum_{k=0}^{\infty} \frac{40 k+3}{112^{2 k}}\left(\begin{array}{c}
4 k \\
2 k
\end{array}\right)\left(\begin{array}{c}
2 k \\
k
\end{array}\right) T_{k}(98,1)=\frac{70 \sqrt{21}}{9 \pi}, \\
& \sum_{k=0}^{\infty} \frac{80 k+9}{264^{2 k}}\left(\begin{array}{c}
4 k \\
2 k
\end{array}\right)\left(\begin{array}{c}
2 k \\
k
\end{array}\right) T_{k}(257,256)=\frac{11 \sqrt{66}}{2 \pi} \\
& \sum_{k=0}^{\infty} \frac{80 k+13}{\left(-168^{2}\right)^{k}}\left(\begin{array}{c}
4 k \\
2 k
\end{array}\right)\left(\begin{array}{c}
2 k \\
k
\end{array}\right) T_{k}(7,4096)=\frac{14 \sqrt{210}+21 \sqrt{42}}{8 \pi}, \\
& \sum_{k=0}^{\infty} \frac{760 k+71}{336^{2 k}}\left(\begin{array}{c}
4 k \\
2 k
\end{array}\right)\left(\begin{array}{c}
2 k \\
k
\end{array}\right) T_{k}(322,1)=\frac{126 \sqrt{7}}{\pi}, \\
& \sum_{k=0}^{\infty} \frac{10 k-1}{336^{2 k}}\left(\begin{array}{c}
4 k \\
2 k
\end{array}\right)\left(\begin{array}{c}
2 k \\
k
\end{array}\right) T_{k}(1442,1)=\frac{7 \sqrt{210}}{4 \pi} \\
& \sum_{k=0}^{\infty} \frac{770 k+69}{912^{2 k}}\left(\begin{array}{c}
4 k \\
2 k
\end{array}\right)\left(\begin{array}{c}
2 k \\
k
\end{array}\right) T_{k}(898,1)=\frac{95 \sqrt{114}}{4 \pi}, \\
& \sum_{k=0}^{\infty} \frac{280 k-139}{912^{2 k}}\left(\begin{array}{c}
4 k \\
2 k
\end{array}\right)\left(\begin{array}{c}
2 k \\
k
\end{array}\right) T_{k}(12098,1)=\frac{95 \sqrt{399}}{\pi}, \\
& \sum_{k=0}^{\infty} \frac{84370 k+6011}{10416^{2 k}}\left(\begin{array}{c}
4 k \\
2 k
\end{array}\right)\left(\begin{array}{c}
2 k \\
k
\end{array}\right) T_{k}(10402,1)=\frac{3689 \sqrt{434}}{4 \pi},
\end{aligned}
$$




$$
\begin{gathered}
\sum_{k=0}^{\infty} \frac{8840 k-50087}{10416^{2 k}}\left(\begin{array}{c}
4 k \\
2 k
\end{array}\right)\left(\begin{array}{c}
2 k \\
k
\end{array}\right) T_{k}(1684802,1)=\frac{7378 \sqrt{8463}}{\pi}, \\
\sum_{k=0}^{\infty} \frac{11657240 k+732103}{39216^{2 k}}\left(\begin{array}{c}
4 k \\
2 k
\end{array}\right)\left(\begin{array}{c}
2 k \\
k
\end{array}\right) T_{k}(39202,1)=\frac{80883 \sqrt{817}}{\pi} \\
\sum_{k=0}^{\infty} \frac{3080 k-58871}{39216^{2 k}}\left(\begin{array}{c}
4 k \\
2 k
\end{array}\right)\left(\begin{array}{c}
2 k \\
k
\end{array}\right) T_{k}(23990402,1)=\frac{17974 \sqrt{2451}}{\pi} .
\end{gathered}
$$

Remark 5.3. (III12) and (III13) are dual in view of (43). Other dual pairs include (III6) and (III7), (III8) and (III9), (III10) and (III11). Below are the corresponding $p$-adic congruences for (III1) and (III13) (with $p>3$ a prime):

$$
\begin{aligned}
& \sum_{k=0}^{p-1}(85 k+2) \frac{\left(\begin{array}{c}
4 k \\
2 k
\end{array}\right)\left(\begin{array}{c}
2 k \\
k
\end{array}\right) T_{k}(52,1)}{66^{2 k}} \\
\equiv & p\left(12\left(\frac{-33}{p}\right)-10\left(\frac{33}{p}\right)\right)\left(\bmod p^{2}\right)(p \neq 11), \\
& \sum_{k=0}^{p-1}(3080 k-58871) \frac{\left(\begin{array}{c}
4 k \\
2 k
\end{array}\right)\left(\begin{array}{c}
2 k \\
k
\end{array}\right) T_{k}(23990402,1)}{39216^{2 k}} \\
\equiv & p\left(385\left(\frac{-2451}{p}\right)-59256\left(\frac{1634}{p}\right)\right)\left(\bmod p^{2}\right) \quad(p \neq 19,43) .
\end{aligned}
$$

Conjecture IV. We have

$$
\begin{aligned}
\sum_{k=0}^{\infty} \frac{26 k+5}{\left(-48^{2}\right)^{k}}\left(\begin{array}{c}
2 k \\
k
\end{array}\right)^{2} T_{2 k}(7,1) & =\frac{48}{5 \pi}, \\
\sum_{k=0}^{\infty} \frac{340 k+59}{\left(-480^{2}\right)^{k}}\left(\begin{array}{c}
2 k \\
k
\end{array}\right)^{2} T_{2 k}(62,1) & =\frac{120}{\pi}, \\
\sum_{k=0}^{\infty} \frac{13940 k+1559}{\left(-5760^{2}\right)^{k}}\left(\begin{array}{c}
2 k \\
k
\end{array}\right)^{2} T_{2 k}(322,1) & =\frac{4320}{\pi},
\end{aligned}
$$




$$
\begin{aligned}
& \sum_{k=0}^{\infty} \frac{8 k+1}{96^{2 k}}\left(\begin{array}{c}
2 k \\
k
\end{array}\right)^{2} T_{2 k}(10,1)=\frac{10 \sqrt{2}}{3 \pi}, \\
& \sum_{k=0}^{\infty} \frac{10 k+1}{240^{2 k}}\left(\begin{array}{c}
2 k \\
k
\end{array}\right)^{2} T_{2 k}(38,1)=\frac{15 \sqrt{6}}{4 \pi}, \\
& \sum_{k=0}^{\infty} \frac{14280 k+899}{39200^{2 k}}\left(\begin{array}{c}
2 k \\
k
\end{array}\right)^{2} T_{2 k}(198,1)=\frac{1155 \sqrt{6}}{\pi} \\
& \sum_{k=0}^{\infty} \frac{120 k+13}{320^{2 k}}\left(\begin{array}{c}
2 k \\
k
\end{array}\right)^{2} T_{2 k}(18,1)=\frac{12 \sqrt{15}}{\pi}, \\
& \sum_{k=0}^{\infty} \frac{21 k+2}{896^{2 k}}\left(\begin{array}{c}
2 k \\
k
\end{array}\right)^{2} T_{2 k}(30,1)=\frac{5 \sqrt{7}}{2 \pi}, \\
& \sum_{k=0}^{\infty} \frac{56 k+3}{24^{4 k}}\left(\begin{array}{c}
2 k \\
k
\end{array}\right)^{2} T_{2 k}(110,1)=\frac{30 \sqrt{7}}{\pi} \\
& \sum_{k=0}^{\infty} \frac{56 k+5}{48^{4 k}}\left(\begin{array}{c}
2 k \\
k
\end{array}\right)^{2} T_{2 k}(322,1)=\frac{72 \sqrt{7}}{5 \pi} \\
& \sum_{k=0}^{\infty} \frac{10 k+1}{2800^{2 k}}\left(\begin{array}{c}
2 k \\
k
\end{array}\right)^{2} T_{2 k}(198,1)=\frac{25 \sqrt{14}}{24 \pi}, \\
& \sum_{k=0}^{\infty} \frac{195 k+14}{10400^{2 k}}\left(\begin{array}{c}
2 k \\
k
\end{array}\right)^{2} T_{2 k}(102,1)=\frac{85 \sqrt{39}}{12 \pi} \\
& \sum_{k=0}^{\infty} \frac{3230 k+263}{46800^{2 k}}\left(\begin{array}{c}
2 k \\
k
\end{array}\right)^{2} T_{2 k}(1298,1)=\frac{675 \sqrt{26}}{4 \pi} \\
& \sum_{k=0}^{\infty} \frac{520 k-111}{5616^{2 k}}\left(\begin{array}{c}
2 k \\
k
\end{array}\right)^{2} T_{2 k}(1298,1)=\frac{1326 \sqrt{3}}{\pi} \\
& \sum_{k=0}^{\infty} \frac{280 k-149}{20400^{2 k}}\left(\begin{array}{c}
2 k \\
k
\end{array}\right)^{2} T_{2 k}(4898,1)=\frac{330 \sqrt{51}}{\pi} \\
& \sum_{k=0}^{\infty} \frac{78 k-1}{28880^{2 k}}\left(\begin{array}{c}
2 k \\
k
\end{array}\right)^{2} T_{2 k}(5778,1)=\frac{741 \sqrt{10}}{20 \pi} \\
& \sum_{k=0}^{\infty} \frac{57720 k+3967}{439280^{2 k}}\left(\begin{array}{c}
2 k \\
k
\end{array}\right)^{2} T_{2 k}(5778,1)=\frac{2890 \sqrt{19}}{\pi} \\
& \sum_{k=0}^{\infty} \frac{1615 k-314}{243360^{2 k}}\left(\begin{array}{c}
2 k \\
k
\end{array}\right)^{2} T_{2 k}(54758,1)=\frac{1989 \sqrt{95}}{4 \pi}
\end{aligned}
$$


and

$$
\begin{array}{r}
\sum_{k=0}^{\infty} \frac{34 k+5}{4608^{k}}\left(\begin{array}{c}
2 k \\
k
\end{array}\right)^{2} T_{2 k}(10,-2)=\frac{12 \sqrt{6}}{\pi}, \\
\sum_{k=0}^{\infty} \frac{130 k+1}{1161216^{k}}\left(\begin{array}{c}
2 k \\
k
\end{array}\right)^{2} T_{2 k}(238,-14)=\frac{288 \sqrt{2}}{\pi}, \\
\sum_{k=0}^{\infty} \frac{2380 k+299}{(-16629048064)^{k}}\left(\begin{array}{c}
2 k \\
k
\end{array}\right)^{2} T_{2 k}(9918,-19)=\frac{860 \sqrt{7}}{3 \pi} .
\end{array}
$$

Remark 5.4. For (IV6), Mathematica indicates that if we set

$$
s(n):=\sum_{k=0}^{n} \frac{14280 k+899}{39200^{2 k}}\left(\begin{array}{c}
2 k \\
k
\end{array}\right)^{2} T_{2 k}(198,1)
$$

then

$$
\left|s(15) \times \frac{\pi}{1155 \sqrt{6}}-1\right|<\frac{1}{10^{50}} \text { and }\left|s(30) \times \frac{\pi}{1155 \sqrt{6}}-1\right|<\frac{1}{10^{100}} .
$$

Here are corresponding $p$-adic congruences of (IV9)-(IV11) and (IV18) with $p>5$ a prime:

$$
\begin{gathered}
\sum_{k=0}^{p-1}(56 k+3) \frac{\left(\begin{array}{c}
2 k \\
k
\end{array}\right)^{2} T_{2 k}(110,1)}{24^{4 k}} \equiv \frac{p}{4}\left(35\left(\frac{p}{7}\right)-23\right)\left(\bmod p^{2}\right) \\
\sum_{k=0}^{p-1}(56 k+5) \frac{\left(\begin{array}{c}
2 k \\
k
\end{array}\right)^{2} T_{2 k}(322,1)}{48^{4 k}} \equiv \frac{p}{20}\left(147\left(\frac{p}{7}\right)-47\right)\left(\bmod p^{2}\right) \\
\sum_{k=0}^{p-1}(10 k+1) \frac{\left(\begin{array}{c}
2 k \\
k
\end{array}\right)^{2} T_{2 k}(198,1)}{2800^{2 k}} \\
\equiv \frac{p}{12}\left(\frac{2}{p}\right)\left(13\left(\frac{p}{7}\right)-1\right)\left(\bmod p^{2}\right)(p \neq 7)
\end{gathered}
$$

and

$$
\begin{aligned}
& \sum_{k=0}^{p-1}(1615 k-314) \frac{\left(\begin{array}{c}
2 k \\
k
\end{array}\right)^{2} T_{2 k}(54758,1)}{243360^{2 k}} \\
\equiv & \frac{p}{26}\left(6137\left(\frac{p}{95}\right)-14301\right)\left(\bmod p^{2}\right) \quad(p \neq 13) .
\end{aligned}
$$

For any prime $p>3$, the corresponding $p$-adic congruence of (IV19) is

$$
\sum_{k=0}^{p-1} \frac{34 k+5}{4608^{k}}\left(\begin{array}{c}
2 k \\
k
\end{array}\right)^{2} T_{2 k}(10,-2) \equiv p\left(6\left(\frac{-6}{p}\right)-\left(\frac{6}{p}\right)\right)\left(\bmod p^{2}\right) .
$$


Conjecture V. We have the formula

$$
\sum_{k=0}^{\infty} \frac{1638 k+277}{(-240)^{3 k}}\left(\begin{array}{c}
2 k \\
k
\end{array}\right)\left(\begin{array}{c}
3 k \\
k
\end{array}\right) T_{3 k}(62,1)=\frac{44 \sqrt{105}}{\pi}
$$

Remark 5.5. (V1) was motivated by Conjecture 4.10; the series converges at a geometric rate with ratio $-64 / 125$.

We conjecture that (IV1)-(IV18) have exhausted all identities of the form

$$
\sum_{k=0}^{\infty}(a+d k) \frac{\left(\begin{array}{c}
2 k \\
k
\end{array}\right)^{2} T_{2 k}(b, 1)}{m^{k}}=\frac{C}{\pi}
$$

with $a, d, m \in \mathbb{Z}, b \in\{1,3,4, \ldots\}, d>0$, and $C^{2}$ rational. This comes from our following hypothesis motivated by (16) in the case $h=2$ and the author's philosophy about series for $1 / \pi$ stated in [Su7]. We have applied the hypothesis to seek for series for $1 / \pi$ of type IV and checked all those $b=1, \ldots, 10^{6}$ via computer.

Hypothesis 5.1 (i) Suppose that

$$
\sum_{k=0}^{\infty} \frac{a+d k}{m^{k}}\left(\begin{array}{c}
2 k \\
k
\end{array}\right)^{2} T_{2 k}(b, 1)=\frac{C}{\pi}
$$

with $a, d, m \in \mathbb{Z}, b \in \mathbb{Z}^{+}$and $C^{2} \in \mathbb{Q} \backslash\{0\}$. Then $\sqrt{|m|}$ is an integer dividing $16\left(b^{2}-4\right)$. Also, $b=7$ or $b \equiv 2(\bmod 4)$.

(ii) Let $\varepsilon \in\{ \pm 1\}, b, m \in \mathbb{Z}^{+}$and $m \mid 16\left(b^{2}-4\right)$. Then, there are $a, d \in \mathbb{Z}$ such that

$$
\sum_{k=0}^{\infty} \frac{a+d k}{\left(\varepsilon m^{2}\right)^{k}}\left(\begin{array}{c}
2 k \\
k
\end{array}\right)^{2} T_{2 k}(b, 1)=\frac{C}{\pi}
$$

for some $C \neq 0$ with $C^{2}$ rational, if and only if $m>4(b+2)$ and

$$
\sum_{k=0}^{p-1} \frac{\left(\begin{array}{c}
2 k \\
k
\end{array}\right)^{2} T_{2 k}(b, 1)}{\left(\varepsilon m^{2}\right)^{k}} \equiv\left(\frac{\varepsilon\left(b^{2}-4\right)}{p}\right) \sum_{k=0}^{p-1} \frac{\left(\begin{array}{c}
2 k \\
k
\end{array}\right)^{2} T_{2 k}(b, 1)}{\left(\varepsilon \bar{m}^{2}\right)^{k}}\left(\bmod p^{2}\right)
$$

for all odd primes $p \nmid b^{2}-4$, where $\bar{m}=16\left(b^{2}-4\right) / m$. 
Conjecture VI. We have the following formulae:

$$
\begin{aligned}
\sum_{k=0}^{\infty} \frac{66 k+17}{\left(2^{11} 3^{3}\right)^{k}} T_{k}^{3}\left(10,11^{2}\right) & =\frac{540 \sqrt{2}}{11 \pi}, \\
\sum_{k=0}^{\infty} \frac{126 k+31}{(-80)^{3 k}} T_{k}^{3}\left(22,21^{2}\right) & =\frac{880 \sqrt{5}}{21 \pi} \\
\sum_{k=0}^{\infty} \frac{3990 k+1147}{(-288)^{3 k}} T_{k}^{3}\left(62,95^{2}\right) & =\frac{432}{95 \pi}(195 \sqrt{14}+94 \sqrt{2}) .
\end{aligned}
$$

Remark 5.6. The series (VI1)-(VI3) converge at geometric rates with ratios

$$
\frac{16}{27},-\frac{64}{125},-\frac{343}{512}
$$

respectively. The author would like to offer $\$ 300$ as the prize for the person (not joint authors) who can provide first rigorous proofs of all the three identities (VI1)-(VI3). (VI1) and (VI3) were motivated by the author's following conjectural congruences for any prime $p>3$ :

$$
\begin{gathered}
\sum_{k=0}^{p-1} \frac{T_{k}^{3}\left(10,11^{2}\right)}{\left(2^{11} 3^{3}\right)^{k}} \equiv \begin{cases}\left(\frac{2}{p}\right)\left(4 x^{2}-2 p\right)\left(\bmod p^{2}\right) & \text { if } p=x^{2}+3 y^{2} \\
0\left(\bmod p^{2}\right) & \text { if } p \equiv 2(\bmod 3)\end{cases} \\
\sum_{k=0}^{p-1} \frac{66 k+17}{\left(2^{11} 3^{3}\right)^{k}} T_{k}^{3}\left(10,11^{2}\right) \equiv \frac{p}{11}\left(195\left(\frac{-2}{p}\right)-8\left(\frac{-6}{p}\right)\right)\left(\bmod p^{2}\right) ; \\
\sum_{k=0}^{p-1} \frac{T_{k}^{3}\left(62,95^{2}\right)}{(-288)^{3 k}} \equiv \begin{cases}\left(\frac{-2}{p}\right)\left(4 x^{2}-2 p\right)\left(\bmod p^{2}\right) & \text { if } p=x^{2}+7 y^{2}, \\
0\left(\bmod p^{2}\right) & \text { if }\left(\frac{p}{7}\right)=-1,\end{cases} \\
\sum_{k=0}^{p-1} \frac{3990 k+1147}{(-288)^{3 k}} T_{k}^{3}\left(62,95^{2}\right) \\
\equiv \frac{p}{19}\left(17563\left(\frac{-14}{p}\right)+4230\left(\frac{-2}{p}\right)\right) \\
\left(\bmod p^{2}\right) .
\end{gathered}
$$


Conjecture VII. We have the following formulae:

$$
\begin{aligned}
\sum_{k=0}^{\infty} \frac{221 k+28}{450^{k}}\left(\begin{array}{c}
2 k \\
k
\end{array}\right) T_{k}^{2}(6,2) & =\frac{2700}{7 \pi}, \\
\sum_{k=0}^{\infty} \frac{24 k+5}{28^{2 k}}\left(\begin{array}{c}
2 k \\
k
\end{array}\right) T_{k}^{2}(4,9) & =\frac{49}{9 \pi}(\sqrt{3}+\sqrt{6}), \\
\sum_{k=0}^{\infty} \frac{560 k+71}{22^{2 k}}\left(\begin{array}{c}
2 k \\
k
\end{array}\right) T_{k}^{2}(5,1) & =\frac{605 \sqrt{7}}{3 \pi}, \\
\sum_{k=0}^{\infty} \frac{3696 k+445}{46^{2 k}}\left(\begin{array}{c}
2 k \\
k
\end{array}\right) T_{k}^{2}(7,1) & =\frac{1587 \sqrt{7}}{2 \pi} \\
\sum_{k=0}^{\infty} \frac{56 k+19}{(-108)^{k}}\left(\begin{array}{c}
2 k \\
k
\end{array}\right) T_{k}^{2}(3,-3) & =\frac{9 \sqrt{7}}{\pi}, \\
\sum_{k=0}^{\infty} \frac{450296 k+53323}{(-5177196)^{k}}\left(\begin{array}{c}
2 k \\
k
\end{array}\right) T_{k}^{2}(171,-171) & =\frac{113535 \sqrt{7}}{2 \pi}, \\
\sum_{k=0}^{\infty} \frac{2800512 k+435257}{434^{2 k}}\left(\begin{array}{c}
2 k \\
k
\end{array}\right) T_{k}^{2}(73,576) & =\frac{10406669}{2 \sqrt{6} \pi} .
\end{aligned}
$$

Remark 5.7. The series (VII1)-(VII7) converge at geometric rates with ratios

$$
\frac{88+48 \sqrt{2}}{225}, \frac{25}{49}, \frac{49}{121}, \frac{81}{529},-\frac{7}{9},-\frac{175}{7569}, \frac{14641}{47089} .
$$

respectively. The author found (VII2) and (VII3) in light of Conjecture 4.18. Similarly, (VII6)-(VII7) were motivated by Conjectures 4.19-4.20.

Concerning the new identities in Conjectures I-VII, actually we first discovered congruences without linear parts related to binary quadratic forms (like many congruences in Section 4), then found corresponding $p$-adic congruences with linear parts, and finally figured out the series for $1 / \pi$.

Acknowledgments. The work was supported by the National Natural Science Foundation (grant 11171140) of China, and the initial version of this paper was posted to arXiv in Jan. 2011 as a preprint with the ID arXiv:1101.0600. The preprint version of this paper available from arXiv has stimulated some others to work on our conjectural series for $1 / \pi$ of types I-V in Section 5. 


\section{REFERENCES}

[AKP] G. Almkvist, C. Krattenthaler and J. Petersson, Some new formulas for $\pi$, Experiment. Math. 12 (2003), 441-456.

[BB] N. D. Baruah and B. C. Berndt, Eisenstein series and Ramanujan-type series for $1 / \pi$, Ramanujan J. 23 (2010), 17-44.

[Be] B. C. Berndt, Ramanujan's Notebooks, Part IV, Springer, New York, 1994.

[BEW] B. C. Berndt, R. J. Evans and K. S. Williams, Gauss and Jacobi Sums, John Wiley \& Sons, 1998.

[CHV] J. S. Caughman, C. R. Haithcock and J. J. P. Veerman, A note on lattice chains and Delannoy numbers, Discrete Math. 308 (2008), 2623-2628.

[CCL] H. H. Chan, S. H. Chan and Z.-G. Liu, Domb's numbers and RamanujanSato type series for $1 / \pi$, Adv. in Math. 186 (2004), 396-410.

[CC] H. H. Chan and S. Cooper, Rational analogues of Ramanujan's series for 1/ $\pi$, Math. Proc. Cambridge Philos. Soc. 153 (2012), 361-383.

[CDE] S. Chowla, B. Dwork and R. J. Evans, On the mod $p^{2}$ determination of $\left(\begin{array}{c}(p-1) / 2 \\ (p-1) / 4\end{array}\right)$, J. Number Theory 24 (1986), 188-196.

[ChCh] D. V. Chudnovsky and G. V. Chudnovsky, Approximations and complex multiplication according to Ramanujan, in: Ramanujan Revisited: Proc. of the Centenary Confer. (Urbana-Champaign, ILL., 1987), (eds., G. E. Andrews, B. C. Berndt and R. A. Rankin), Academic Press, Boston, MA, 1988, pp. $375-472$.

[C] S. Cooper, Sporadic sequences, modular forms and new series for $1 / \pi$, Ramanujan J. 29 (2012), 163-183.

[Co] D. A. Cox, Primes of the Form $x^{2}+n y^{2}$, John Wiley \& Sons, 1989.

[HW] R. H. Hudson and K. S. Williams, Binomial coefficients and Jacobi sums, Trans. Amer. Math. Soc. 281 (1984), 431-505.

[I] T. Ishikawa, Super congruence for the Apéry numbers, Nagoya Math. J. 118 (1990), 195-202.

[IR] K. Ireland and M. Rosen, A Classical Introduction to Modern Number Theory, 2nd Edition, Springer, New York, 1990.

[L] L. Long, Hypergeometric evaluation identities and supercongruences, Pacific J. Math. 249 (2011), 405-418.

[M1] E. Mortenson, Supercongruences between truncated ${ }_{2} F_{1}$ by geometric functions and their Gaussian analogs, Trans. Amer. Math. Soc. 355 (2003), 987-1007.

[M2] E. Mortenson, A p-adic supercongruence conjecture of van Hamme, Proc. Amer. Math. Soc. 136 (2008), 4321-4328.

[N] T. D. Noe, On the divisibility of generalized central trinomial coefficients, J. Integer Seq. 9 (2006), Article 06.2.7, 12pp.

[PWZ] M. Petkovšek, H. S. Wilf and D. Zeilberger, $A=B$, A K Peters, Wellesley, 1996.

[R] S. Ramanujan, Modular equations and approximations to $\pi$, Quart. J. Math. (Oxford) 45 (1914), 350-372.

[Ri] P. Ribenboim, The Book of Prime Number Records, Second Edition, Springer, New York, 1989.

[RV] F. Rodriguez-Villegas, Hypergeometric families of Calabi-Yau manifolds, in: Calabi-Yau Varieties and Mirror Symmetry (Toronto, ON, 2001), pp. 
223-231, Fields Inst. Commun., 38, Amer. Math. Soc., Providence, RI, 2003.

[Sl] N. J. A. Sloane, Sequence A001850 in OEIS (On-Line Encyclopedia of Integer Sequences), http://www . oeis.org.

[St] R. P. Stanley, Enumerative Combinatorics, Vol. 2, Cambridge Univ. Press, Cambridge, 1999.

[S1] Z.-H. Sun, Congruences concerning Legendre polynomials, Proc. Amer. Math. Soc. 139 (2011), 1915-1929.

[S2] Z.-H. Sun, Congruences concerning Legendre polynomials (II), preprint, arXiv:1012.3898v2 (August 3, 2012).

[S3] Z.-H. Sun, Congruences concerning Legendre polynomials (III), preprint, arXiv:1012.4234.

[S4] Z.-H. Sun, Congruences involving $\left(\begin{array}{c}2 k \\ k\end{array}\right)^{2}\left(\begin{array}{c}3 k \\ k\end{array}\right)$, J. Number Theory 133 (2013), 1572-1595.

[Su1] Z.-W. Sun, Binomial coefficients, Catalan numbers and Lucas quotients, Sci. China Math. 53 (2010), 2473-2488.

[Su2] Z.-W. Sun, On congruences related to central binomial coefficients, J. Number Theory 131 (2011), 2219-2238.

[Su3] Z.-W. Sun, On Delannoy numbers and Schröder numbers, J. Number Theory 131 (2011), 2387-2397.

[Su4] Z.-W. Sun, Super congruences and Euler numbers, Sci. China Math. 54 (2011), 2509-2535.

[Su5] Z.-W. Sun, On sums involving products of three binomial coefficients, Acta Arith. 156 (2012), 123-141.

[Su6] Z.-W. Sun, A refinement of a congruence result by van Hamme and Mortenson, Illinois J. Math., 56 (2012), 967-979.

[Su7] Z.-W. Sun, Conjectures and results on $x^{2} \bmod p^{2}$ with $4 p=x^{2}+d y^{2}$, in: Number Theory and Related Area (eds., Y. Ouyang, C. Xing, F. Xu and P. Zhang), Higher Education Press \& International Press, Beijing and Boston, 2013, 149-197.

[Su8] Z.-W. Sun, Supercongruences involving products of two binomial coeffcients, Finite Fields Appl. 22 (2013), 24-44.

[Su9] Z.-W. Sun, Congruences involving generalized central trinomial coefficients, Sci. China Math. 57 (2014), 1375-1400.

[Sz] G. Szegö, Orthogonal Polynomials, 4th Edition, Amer. Math. Soc., Providence, RI, 1975.

[vH] L. van Hamme, Some conjectures concerning partial sums of generalized hypergeometric series, in: p-adic Functional Analysis (Nijmegen, 1996), pp. 223-236, Lecture Notes in Pure and Appl. Math., Vol. 192, Dekker, 1997.

Department of Mathematics, Nanjing University, Nanjing 210093, PeOple's Republic of ChinA

E-mail address: zwsun@nju.edu.cn 